\title{
GALVANIC VESTIBULAR STIMULATION APPLIED TO FLIGHT TRAINING
}

\author{
A Thesis \\ presented to \\ the Faculty of the College of Engineering \\ California Polytechnic State University \\ In Partial Fulfillment \\ of the Requirements for the Degree \\ Master of Science in Electrical Engineering
}

By

Joel Hanson

July 2009 
(C) 2009

\section{Joel Daniel Hanson}

ALL RIGHTS RESERVED 


\section{APPROVAL PAGE}

TITLE:

Galvanic Vestibular Stimulation Applied to Flight Training

AUTHOR: Joel Daniel Hanson

DATE SUBMITTED: $\quad$ September 2009

COMMITTEE CHAIR: Dr. Lynne Slivovsky, Electrical Engineering Associate Professor

COMMITTEE MEMBER: Dr. Brian Self, Mechanical Engineering Professor

COMMITTEE MEMBER: Dr. Fred DePiero, Professor and Associate Dean for Undergraduate Programs and Curriculum Innovation 


\section{ABSTRACT \\ Galvanic Vestibular Stimulation \\ Applied to flight Training Joel Hanson}

Between 1994 and 2003 spatial disorientation resulted in at least 202 aircraft accidents, 184 of them resulting in fatalities. Galvanic Vestibular Stimulation (GVS) provides a cost effective and safe way to simulate spatial disorientation and potentially test pilot's vestibular system prior to aircraft operation. This experiment investigates the use of GVS as an indicator of motion sensitivity and explores the effects of GVS on flight simulation performance. Bilateral bipolar rectangular pulse electrical stimulations were given to subjects via skin-mounted electrodes placed over each mastoid process to stimulate the vestibular system with roll and yaw sensations. Two studies were conducted in this work: one on motion sensitivity and one to examine the effects of GVS during flight simulator use.

The motion sensitivity test consisted of an alternating GVS sequence to simulate rolling back and forth. The sequence did generate motion sickness in two out of 12 subjects. Results show no correlation to motion history scores calculated from Kennedy’s Motion Sickness Questionnaire (MSQ).

The flight simulator test coupled automatically generated congruent, conflicting, and sham orientation sensations to the roll angles of the aircraft. The stimulations used in this test did not indicate any effect on the simulator flight performance of the subjects. Feedback from subjects during this test raised concern over the delay between left- and right-side stimuli. Further testing to reduce the surface skin sensation showed that a ramp or increasing exponential waveform not only reduced 
the sensation of current entering the body but significantly increased the orientation sensations resulting from the stimulation. Increasing the orientation response and decreasing the sensation of current breaking the surface of the skin provides a much more desired stimulation for each of the tests in this experiment and any other future tests related to GVS. 
Dedicated to Alecia, Andrea, Yvonne, and Jerrold;

My loving and ever supporting family.

Special thanks to Dr Slivovsky and Dr. Self for all your help and guidance. 


\section{Table of Contents}

CHAPTER 1 : Introduction to Galvanic Vestibular Stimulation .................................... 1

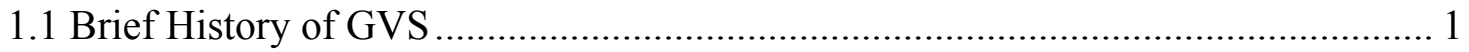

1.2 The Vestibular System...................................................................................... 2

1.2.1 Dynamic motion sensing by the semicircular canals ......................................... 3

1.2.2 Static and dynamic motion sensing by the saccule and utricle......................... 4

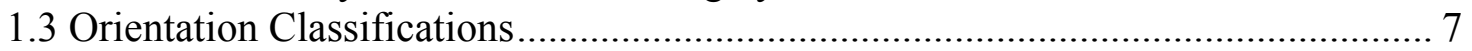

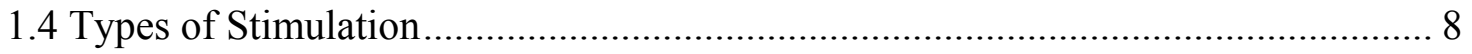

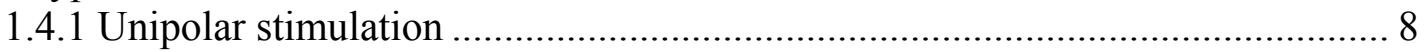

1.4.2 Bilateral bipolar stimulation .................................................................. 9

1.4.3 Bilateral unipolar stimulation ............................................................. 9

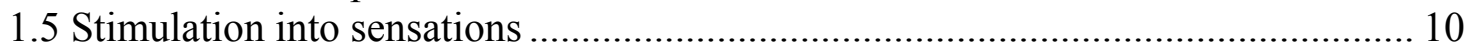

CHAPTER 2 : Electricity and the Human Body ............................................................ 13

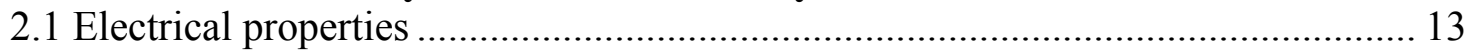

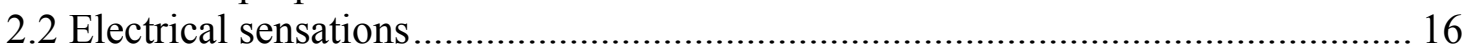

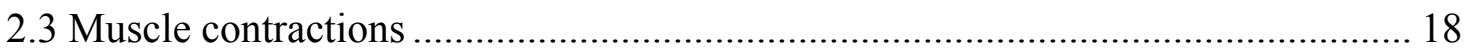

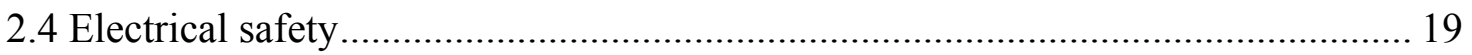

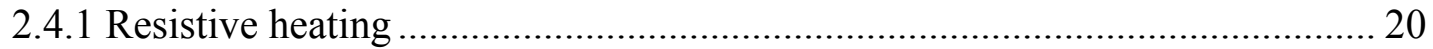

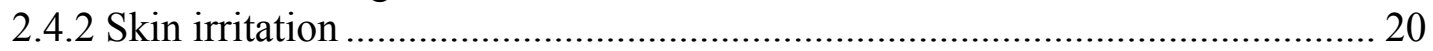

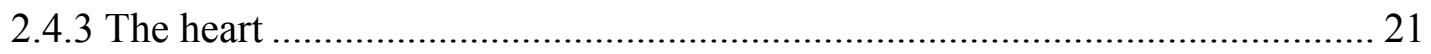

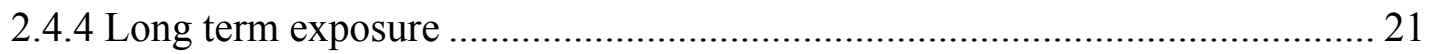

CHAPTER 3 : GVS Literature Review ......................................................................... 23

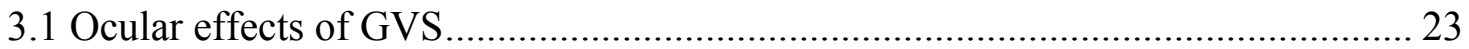

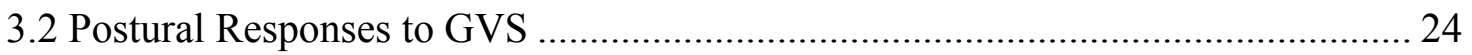

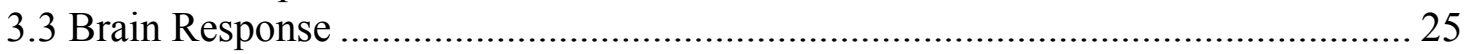

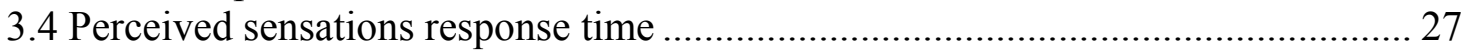

3.5 Relation between literature review and our work ………...................................... 28

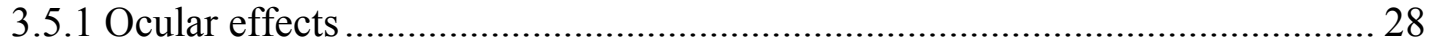

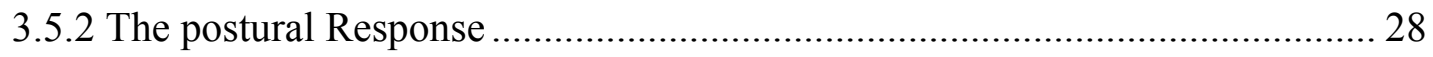

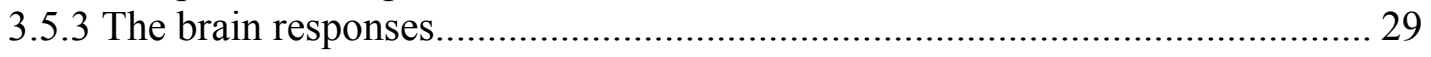

3.5.4 Perceived sensation response time............................................................... 29

CHAPTER 4 : System Design ........................................................................................... 30

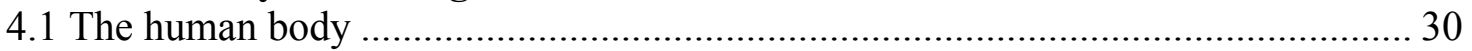

4.2 Galvanic Vestibular Stimulator (GVS) Device.................................................... 32

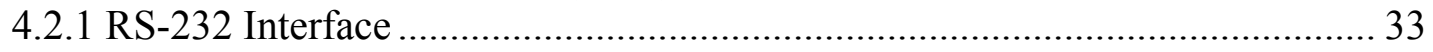

4.2.2 Microprocessor Messages and Commands ..................................................... 33

4.2.3 Digital Signal into Electrical Stimulation ...................................................... 34

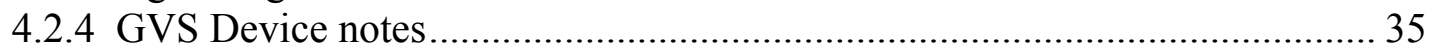

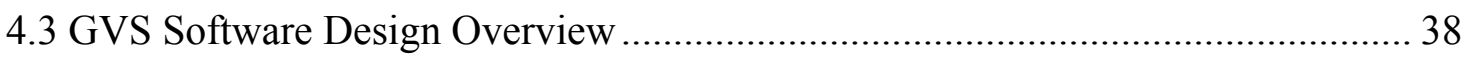

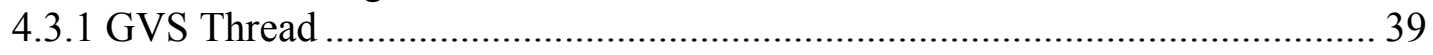

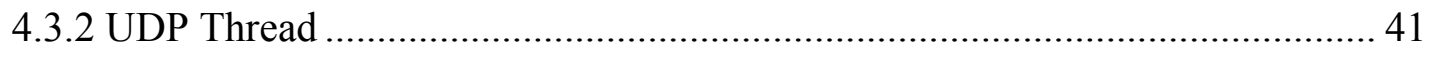

4.4 Motion Sensitivity Test System Design.............................................................. 42

4.5 X-Plane Flight Simulator System Design ............................................................. 47

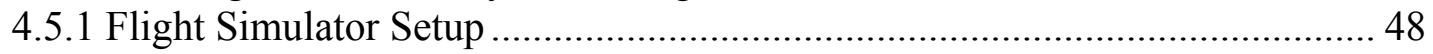

4.5.2 X-Plane Maneuver Series .............................................................................. 49 
4.5.3 Congruent, Conflicting, and Sham Stimulation Profiles ............................. 50

4.5.4 X-Plane Cirrus Jet and Instrument Panel ......................................................... 56

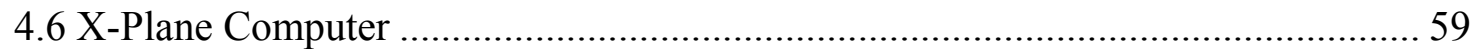

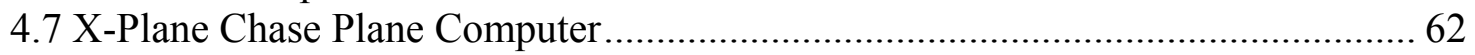

CHAPTER 5 : Experimental Protocol.................................................................64 64

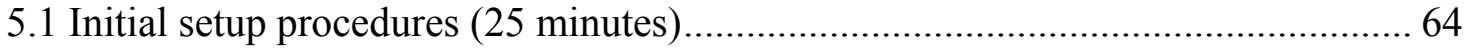

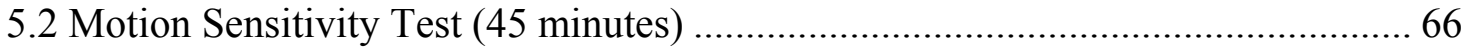

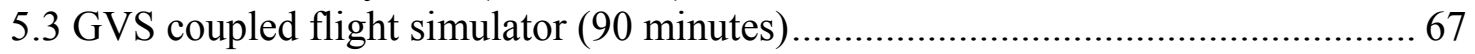

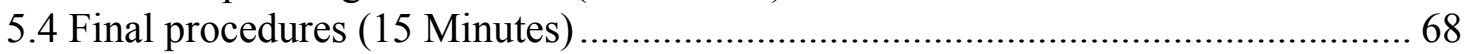

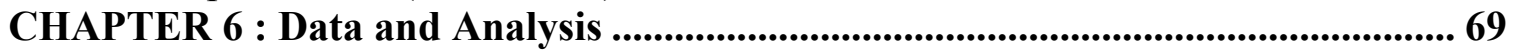

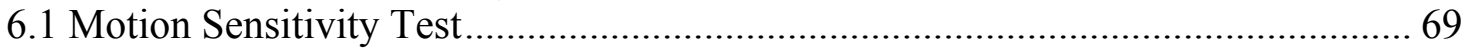

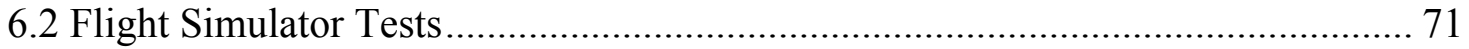

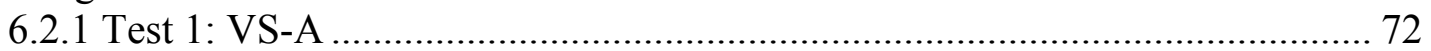

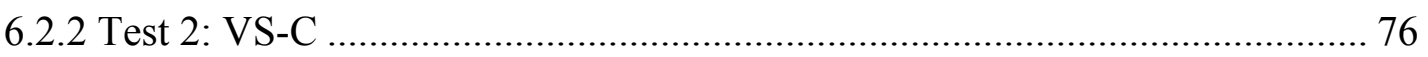

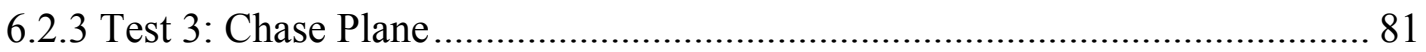

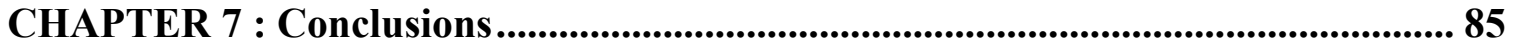

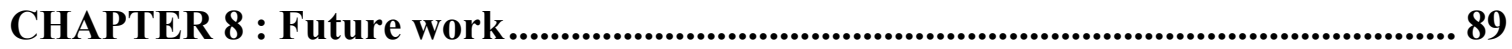

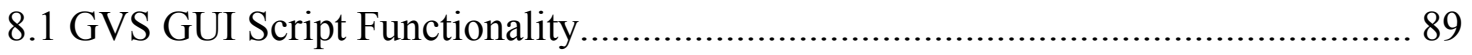

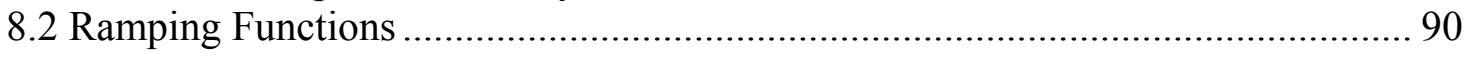

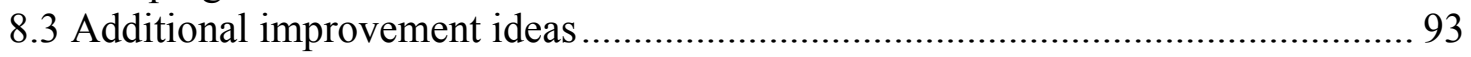

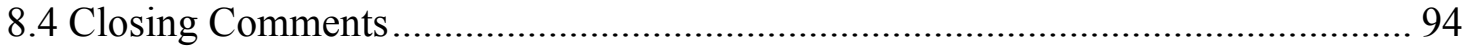

Appendix I - Informed Consent Form for Cal Poly Research ................................... 95

Appendix II - Motion Sickness Screening Questionnaire.......................................... 97

Appendix III - Test Administrator Checklist .............................................................. 100

Appendix IV - Simulator Sickness Questionnaire................................................. 101

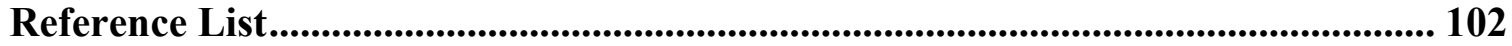

\section{Table of Figures}

FIGURE 1 -INNER EAR STRUCTURE INCLUDING THE SEMICIRCULAR CANALS AND OTOLITH ORGANS, WHICH DETECT ROTATIONAL AND TRANSLATIONAL HEAD MOTIONS RESPECTIVELY [5]....

FIgURE 2 - CUPULA RESPONSE TO A ROTATING HEAD. THE ENDOLYMPH FLUID INSIDE THE SEMICIRCULAR CANAL LAGS THE HEAD ROTATION, CAUSING A DEFLECTION IN THE CUPULA TO SENSE A CHANGE IN HEAD MOTION [24]........................... 4

FIGURE 3 - MACULA AND OTOLITH RESPONSE TO HEAD NOD. AS THE HEAD TILTS FORWARD THE FORCE OF GRAVITY PULLS THE OTOLITHIC MASSES, WHICH DEFLECT THE HAIR CELLS IN THE MACULA AND SENSE A CHANGE IN ORIENTATION HAS TAKEN

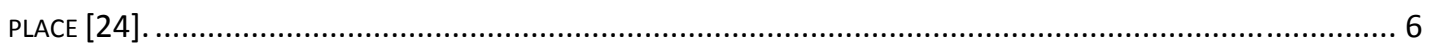

Figure 4 - YAW, PITCH, AND ROLL DIAGRAM. PITCH SENSATIONS ARE FORWARD AND BACKWARD ABOUt THE RIGHT-LEFT AXIS. ROLL SENSATIONS ARE RIGHT AND LEFT AROUND THE FRONT-BACK AXIS. YAW SENSATIONS ARE LEFT AND RIGHT AROUND THE UP-DOWN AXIS [5].

FigURE 5 - ElECTRODE PLACEMENT FOR SENSATIONS OF YAW, PITCH, AND ROLL. ELECTRODE 1 AND ELECTRODE 3 GENERATE THE ROLL SENSATIONS. ELECTRODE 2 AND ELECTRODE 4 GENERATE PITCH SENSATIONS............................................ 8

FIGURE 6 - FITZPATRICK'S YAW, PITCH, AND ROLL SENSATIONS FROM BILATERAL BIPOLAR VESTIBULAR STIMULATIONS. WITH THE ANODE CONNECTED TO THE RIGHT SIDE OF THE BODY AND THE CATHODE CONNECTED TO THE LEFT SIDE OF THE BODY, THE STIMULATION GENERATES SLIGHT YAW TOWARDS THE LEFT SIDE OF THE BODY, ROLL SENSATION TOWARDS THE LEFT SIDE OF BODY, AND NO PITCH SENSATIONS [8].

FiguRE 7 - IMPEDANCE MAGNITUDE AS A FUNCTION OF FREQUENCY. THE PLOTS ARE FOR A MALE, FEMALE, AND A TEN-YEAR-

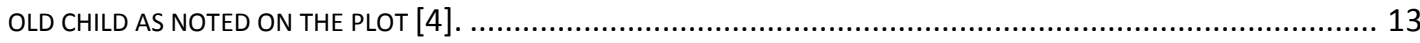

FIGURE 8 - IMPEDANCE PHASE AS A FUNCTION OF FREQUENCY FOR A FEMALE AND A MALE AS NOTED ON THE PLOT [4]........ 14 


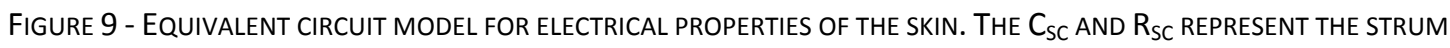
CORNEUM OUTER LAYER OF THE SKIN. RS REPRESENTS THE INNER TISSUES. .............................................. 15

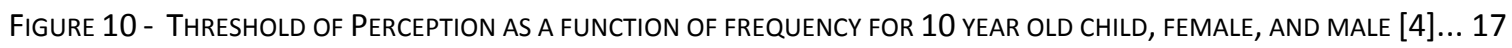

FIGURE 11 - THRESHOLD OF PAIN AS A FUNCTION OF FREQUENCY FOR A TEN-YEAR- OLD CHILD, FEMALE, AND MALE [4]....... 18

FIGURE 12 - ELECTRICAL SAFETY CURRENT THRESHOLDS FOR A $60 \mathrm{HZ}$ SIN WAVE. THRESHOLDS ARE FOR ELECTRODES CONNECTED

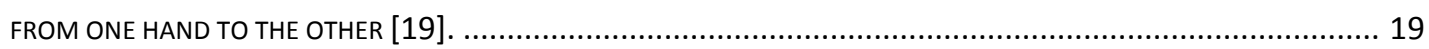

FIGURE 13 -WALKING TRAJECTORY VESTIBULAR STIMULATION DATA. X1 SHOWS THE WALKING TRAJECTORY AT STIMULATIONS OF 1 TIMES THE THRESHOLD CURRENT. X2 WAS DOUBLE THE THRESHOLD, AND X3 WAS TRIPLE THE THRESHOLD CURRENT.

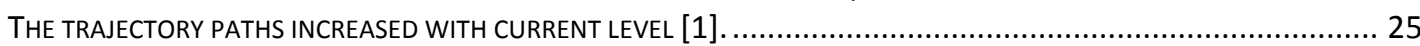

Figure 14 - AREAS OF ACTIVATION IN THE RIGHT, SUPERIOR, AND LEFT HEMISPHERES OF THE BRAIN. THERE WERE SEVEN MAIN

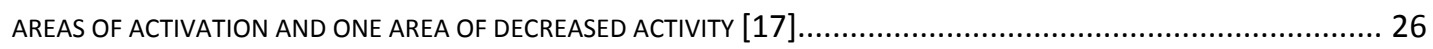

FIGURE 15 - RESPONSE TIME TO GVS, TOUCH, LIGHT, AND SOUND STIMULATIONS [2] .........................................2 27

FIGURE 16 - SYSTEM BLOCK DIAGRAM FOR THE MOTION SENSITIVITY TEST CONSISTING OF THE GVS DEVICE AND THE GVS

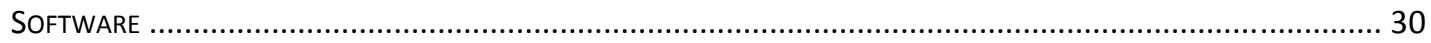

Figure 17 - System block diagram for the X-Plane Virtual Flight Simulator INCLUding the GVS, the GVS SOFTWARE, A COMPUTER RUNNING X-PLANE CONTROlled BY THE SUBJeCt, AND A COMPUTER RUNNING THE X-PLANE

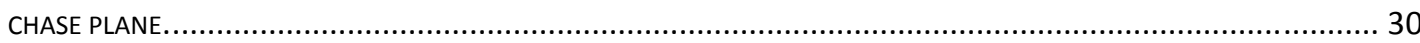

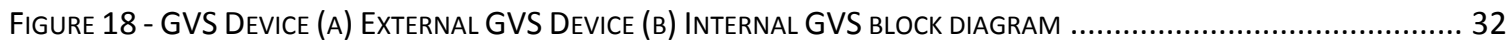

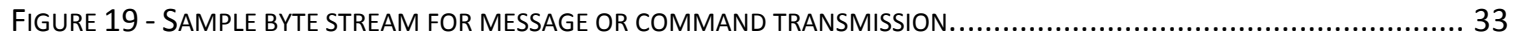

FIGURE 20 - PrECISION VOLTAGE TO CURRENT CONVERTER WITH DIGITAL INPUTS ................................................... 35

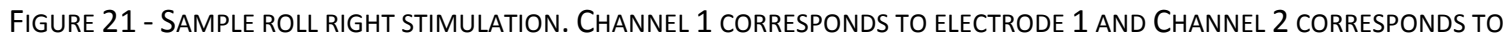
ELECTRODE 3. THE STIMULATION WAS RECORDED AT 1.5 MILLIAMPS ACROSS A $2 \mathrm{~K} \Omega$ RESISTOR. .............................36

Figure 22 - SAMPLE ROLL LEFT STIMULATION WITH NOT FULLY CHARGED BATTERIES. CHANNEL 2 IS ELECTRODE 1 AND

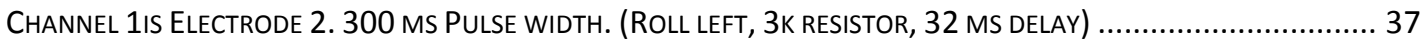

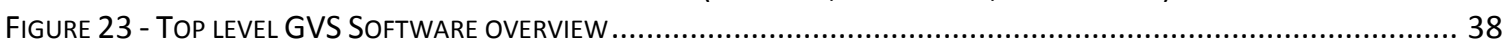

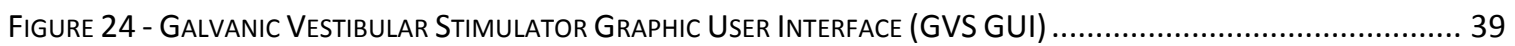

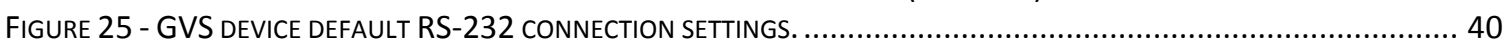

FiguRE 26 - MiddL SECTION OF GUI: OUtPUT FILENAME AND LOCATION AND THE MESSAGE AND COMMAND TEXTBOXES. .. 41

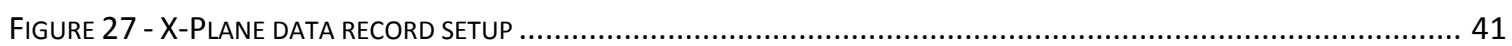

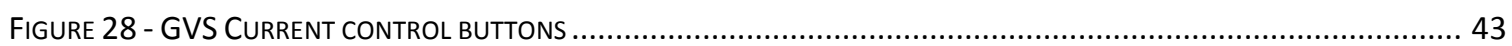

FigURE 29 - Motion SENSITIVITY TEST. BLUE DOT REPRESENTS A ROLL LEFT SENSATION AND A RED DOT REPRESENTS S ROLL

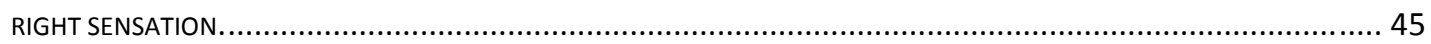

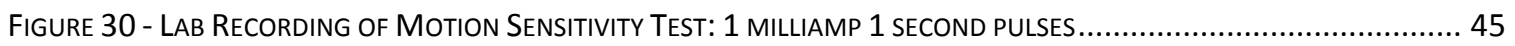

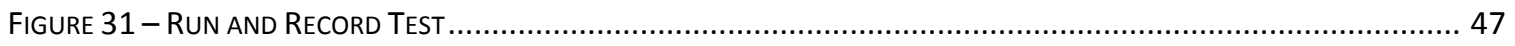

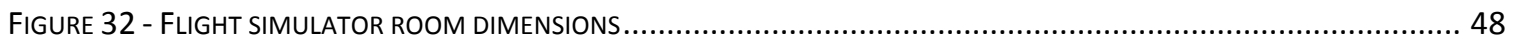

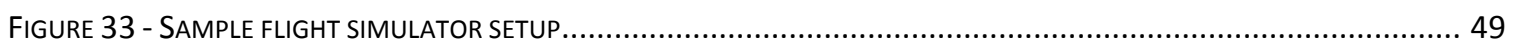

Figure 34 - Roll stimulations generated from Chase Plane test. THe Congruent ANd Conflicting StimULATIONS ARE AT 1.5 MILLIAMPS AND THE SHAM TEST IS AT 0.5 MILLIAMPS. EleCtROde 1 VOLTAGE WAS MEASURED ACROSS A 1 $\mathrm{K} \Omega$ RESISTOR AND ELECTRODE 3 VOLTAGE WAS MEASURED ACROSS A 3 K $\Omega$ RESISTOR. (A) CONGRUENT ROLL Stimulations (B) Conflicting Roll Stimulations (c) SHam Roll Stimulations ...................................... 53

Figure 35 - Pitch stimulations generated from Chase Plane test. The Congruent and Conflicting Stimulations ARE AT 1.5 MILLIAMPS AND THE SHAM TEST IS AT 0.5 MILLIAMPS. ELECTRODE 1 VOLTAGE WAS MEASURED ACROSS A 1 K $\Omega$ RESISTOR AND ELECTRODE 3 VOLTAGE WAS MEASURED ACROSS A 3 K $\Omega$ RESISTOR. (A) CONGRUENT ROLL Stimulations (B) CONfliCting Roll Stimulations (c) SHAM Roll StIMULATIONS ..................................... 55

Figure 36 - CiRRUS Jet USED for AlL OF THE X-PLANE SIMULATOR TESTS ..................................................... 57

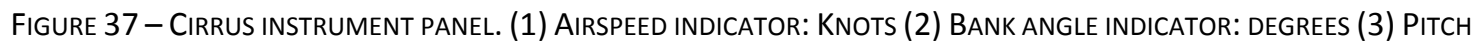

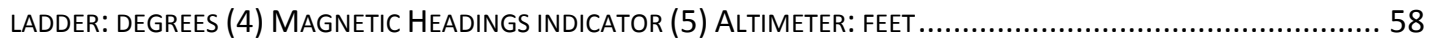

FIGURE 38 - X-PLANE WINDOW FOR ENTERING IN THE IP AND PORT FOR DATA OUTPUT ............................................5 59

FIGURE 39 - X-PLANE SELECTED DATA OUTPUT: SPEEDS, ANGULAR MOMENTS, ANGULAR ACCELERATIONS, ANGULAR VELOCITIES, PITCH, ROLL, HEADINGS, LATITUDE, LONGITUDE, ALTITUDE, LOCATION, VELOCITY, AND DISTANCE TRAVELED..............60

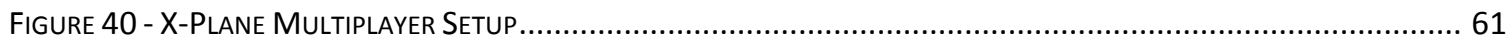

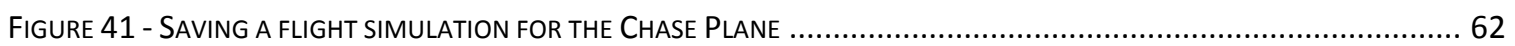

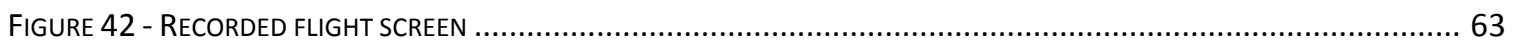


FIGURE 43 - MOTION SICKNESS SCORE ASSOCIATED WITH THE VARYING ROCKING FREQUENCY TESTS

FIGURE 44 - FINAL SICKNESS SCORES OF THE SUBJECTS VERSUS THE MOTION SICKNESS SCORES OF THE SUBJECTS....

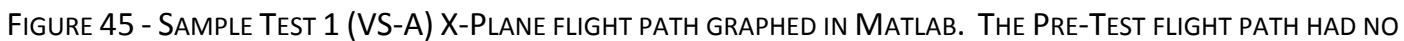

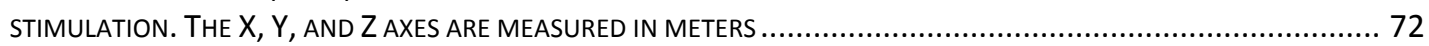

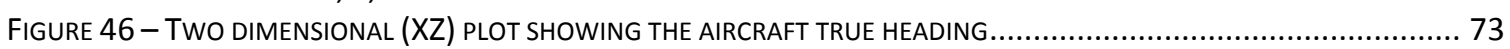

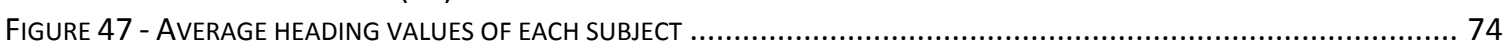

FIGURE 48 - ROOT MEAN SQUARE OF THE DEVIATION OF EACH SUBJECT'S PATH FROM THE DESIRED 0 DEGREES NORTH ......... 75

Figure 49 - SAMPLE Test 2 (VS-C) X-PLANE Flight PATH GRAPHEd IN MATLAB. The Pre-Test Flight PATH had NO

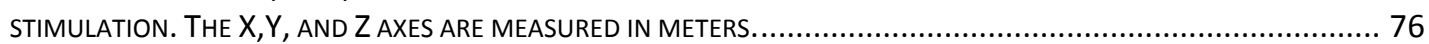

FIGURE 50 -TEST 2 (VS-C) TECHNIQUES FOR EXTRACTING ROLL VALUES FOR THE RMS ROLL DEVIATION CALCULATION. XPLANE WAS SET TO OUTPUT 20 DATA POINTS/SECOND. EVERY 200 POINTS CORRESPONDS TO 10 SECONDS. .................... 78

FIGURE 51 - AVERAGE BANK ANGLE AND RMS DEVIATION OF TEST 2 (VS-C) FOR +30 DEGREE BANK ANGLE .................... 79

FIGURE 52 - AVERAGE BANK ANGLE AND RMS DEVIATION OF TEST 2 (VS-C) FOR -30 DEGREE BANK ANGLE ....................8 80

FIGURE 53 - RMS DEVIATION OF +/- 30 DEGREE BANK ANGLE FOR TEST 2 (VS-C) ............................................. 81

Figure 54 - Sample Test 3 (Chase Plane) X-Plane flight path graphed in Matlab. The Pre-Test flight Path had no STIMULATION. THE $X, Y$, AND Z AXES ARE MEASURED IN METERS. 82

FiguRE 55 - SAMPLE TeSt 3 (ChASE PLANE) 2-DIMENSIONAL FLIGHT PATHS VIEWED FROM LAYING ON THE GROUND LOOKING UP. 83

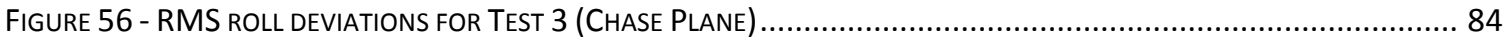

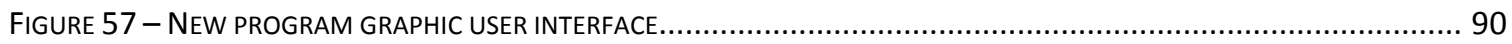

FIGURE 58 - GVS SOFTWARE GENERATED LOGARITHMIC WAVEFORM STIMULATION. THE WAVEFORM HAD A 1.5 MA CURRENT ACROSS A 2 KS RESISTOR.

FIGURE 59-SCRIPT GENERATED LINEAR RAMPING WAVEFORM STIMULATION. THE WAVEFORM HAD A 1.5 MA CURRENT ACROSS A 2 KS RESISTOR.

FIGURE 60 - CYCLES OF A NERVE CELL [24]...... 


\section{CHAPTER 1 : Introduction to Galvanic Vestibular Stimulation}

Spatial disorientation (SD) is "The mistaken perception of one's position and motion relative to the earth" [21]. Between 1994 and 2003, spatial disorientation resulted in at least 202 aircraft accidents, 184 of them resulting in fatalities [21]. What causes people to become disoriented? Are certain people more susceptible to SD than others? Are there ways to safely and cost efficiently test someone's response to disorientation? Could this testing reduce the number of lethal aircraft accidents?

Galvanic vestibular stimulation (GVS) provides a safe, cost effective way to simulate spatial disorientation. Researchers at Cal Poly, in coordination with the Aerospace Medicine \& Vestibular Research Laboratory (AMVRL) at Mayo Clinic in Arizona, have outlined two research objectives to explore in this study. The first objective investigates whether GVS can be used as a test for individual susceptibility to motion sickness. The second objective is to explore the effects of GVS coupled with a visual stimulus (presented by a flight simulator) on the human vestibular system. Each of these objectives has the potential application for safe and cost effective testing of a future pilot's vestibular system and applications in pilot training methods.

\subsection{Brief History of GVS}

Galvanic Vestibular Stimulation uses electricity to stimulate the vestibular system. The study of GVS began in the late eighteenth century when Alessandro Volta reported feeling dizziness after he applied current across his head [26]. 
Volta, inventor of the battery, put electrodes attached to approximately a 30 volt source in his ears, resulting in what he described as "An explosion inside of his head, including loud noises and disorientation" [8]. In 1820 the first formal reporting that galvanic stimulations upset balance and equilibrium was provided by Bohemian physiologist Johann Purkyne in his dissertation [8]. Since this time GVS has been used as a technique to study the effects of applied current on the vestibular system.

\subsection{The Vestibular System}

Although the brain uses many sensory inputs to maintain spatial equilibrium, the dizziness or unbalanced sensation that Volta felt was generated by the vestibular system. The equilibrium and balance receptor organs triggered in GVS are called the vestibular apparatus, which includes the semicircular canals, the saccule, and the utricle shown in Figure 1. Equilibrium and balance can be broken down into static equilibrium, maintenance of the head position in response to gravity, and dynamic equilibrium, maintenance of the head position in response to rotation, acceleration, and deceleration [24]. The three semicircular canals, the saccule, and the utricle function together to provide static and dynamic sensory orientation information. 


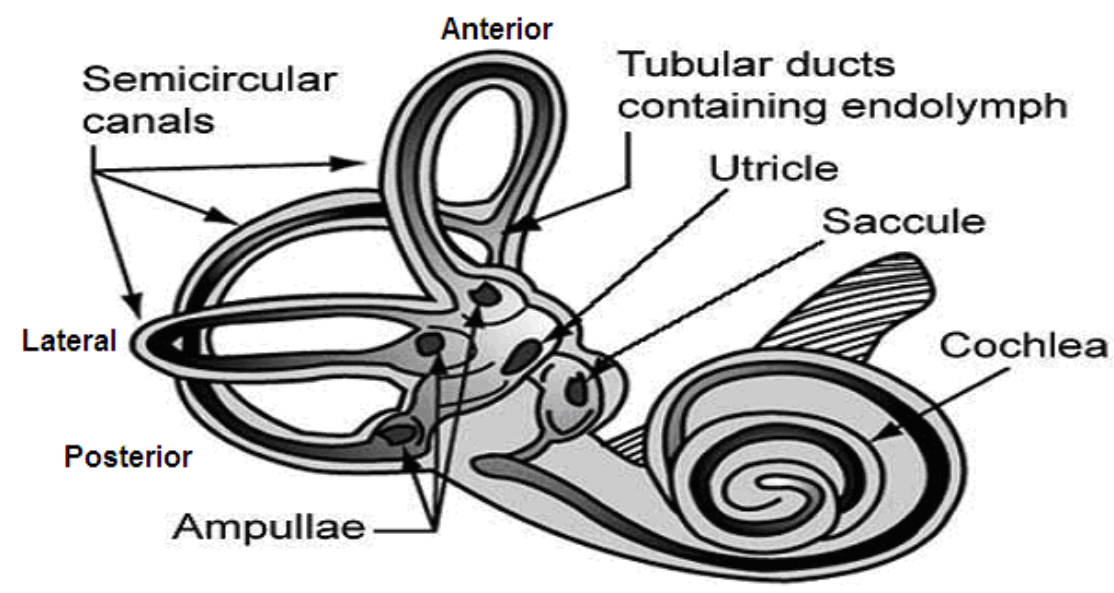

Figure 1 -Inner ear structure including the semicircular canals and otolith organs, which detect rotational and translational head motions respectively [5].

\subsubsection{Dynamic motion sensing by the semicircular canals}

The semicircular canals provide dynamic rotational sensory orientation information. At the base of each semicircular duct lies a dilation of the canal called an ampulla [24]. Located inside of each ampulla are crista. The crista are composed of hair cells and supporting sensory cells covered by a viscous gel-like substance called the cupula [5]. Movement of the head causes the semicircular ducts and hair cells to move with it. The lagging endolymph, fluid within the membranous labyrinth of the inner ear [24], causes the hair bundles to move within the cupula, which allows the change in acceleration to be sensed by the hair cells. This hair deflection causes a depolarization of nerves, and the resulting impulse notifies the person that a change in acceleration has taken place [23]. The deflection of the cupula resulting from a head turn is demonstrated in Figure 2. 


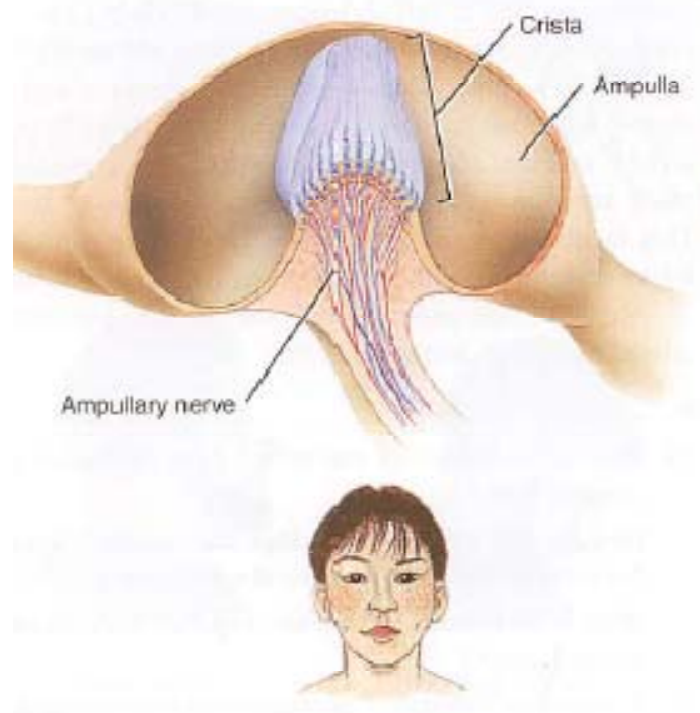

Head in still position

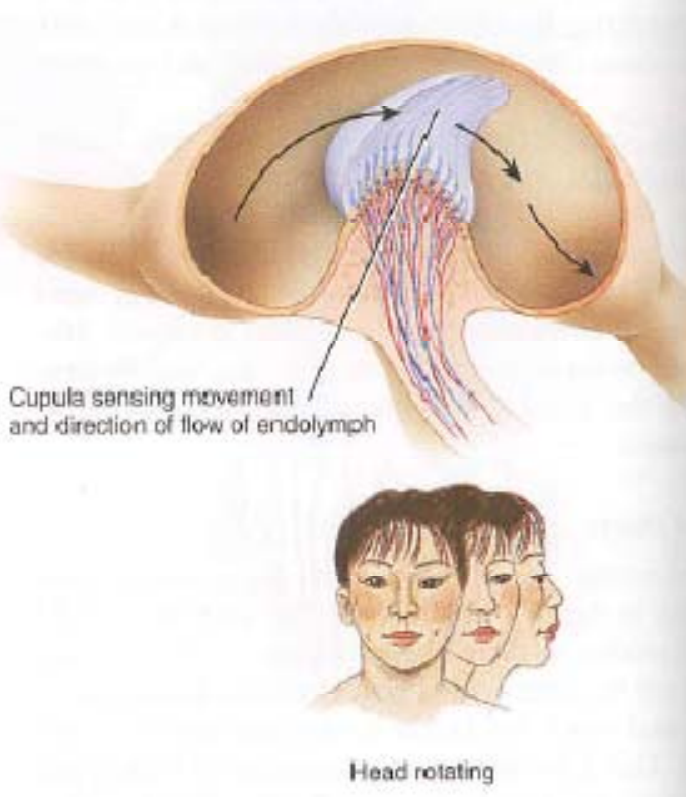

Figure 2 - Cupula response to a rotating head. The endolymph fluid inside the semicircular canal lags the head rotation, causing a deflection in the cupula to sense a change in head motion [24].

The inner ears of the body have three canals: anterior, posterior, and lateral (also known as the horizontal canal). The canals are oriented to detect motion about different axes of the head. For example if a yaw left occurs, the afferent activity from the left horizontal canal increases and the firing rate from the right canal decreases [8]. These inversely related changes in firing rates both contribute to the brain's sensing of the change in yaw that took place.

\subsubsection{Static and dynamic motion sensing by the saccule and utricle}

The walls of the utricle and saccule are composed of small thickened regions covered with macula which has thousands of hairs that detect acceleration.

Residing on the macula, otolithic masses are comprised of a membrane that causes deflections in the hairs. The top of the membrane consists of otoliths, whose specific gravity is about three times that of surrounding tissues [24]. 
Orientation sensations occur when a change in the position of the head causes the weight of the otoliths to bend the membrane and ultimately the bundles of hairs residing in the macula [13]. The utricle and saccule provide static and dynamic translational sensory information [24]. The static information provided indicates the orientation of the head relative to gravity. Figure 3 provides an example of a change in static equilibrium with the tilt of the head down. The dynamic information provided by the utricle and saccule sense when you are speeding up or slowing down. This sensation can be felt in an accelerating or decelerating car or elevator. The sensory information is used to inform ocular and muscle systems that a change in orientation has taken place [26]. 


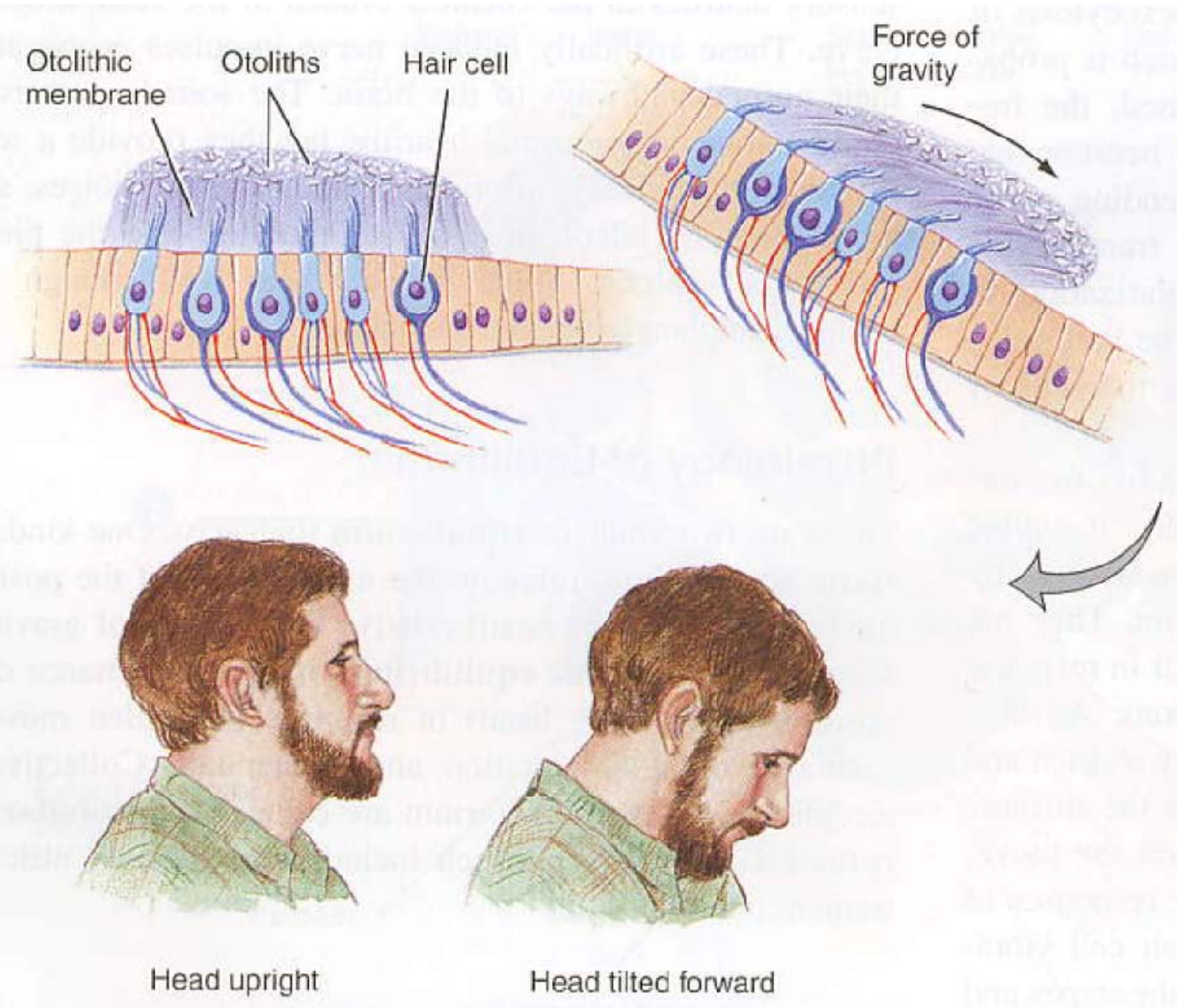

Figure 3 - Macula and otolith response to head nod. As the head tilts forward the force of gravity pulls the otolithic masses, which deflect the hair cells in the macula and sense a change in orientation has taken place [24].

The utricular macula and the saccular macula also reside in different planes for motion detection. The utricular macula is inclined backwards by 30 degrees from the horizontal plane, detecting lateral and saggital motions. The saccular macula is aligned with the saggital or vertical plane, primarily detecting vertical and anteroposterior accelerations. Within both maculae are two regions of reversed hair cell polarity. When a change in acceleration takes place the different regions either increase or decrease firing rates. The brain interprets the area of varying firing rates into motion detection [8]. 


\subsection{Orientation Classifications}

Galvanic Vestibular Stimulation (GVS) is used to stimulate nerves associated with the semi-circular canals, the utricle, and the saccule to generate different orientation sensations. Yaw, pitch, and roll are used to describe the sensations generated from GVS. Imagine a vertical line through the center of the human body; rotating about that vertical axis is yaw. Now imagine horizontal line through the middle of the nose and out of the back of the head; rotating the head about that line results in roll. For the final classification, imagine a horizontal line from one ear to the other, rotating the head about that axis results in a pitch sensation. Figure 4 summarizes the yaw, pitch, and roll sensations.

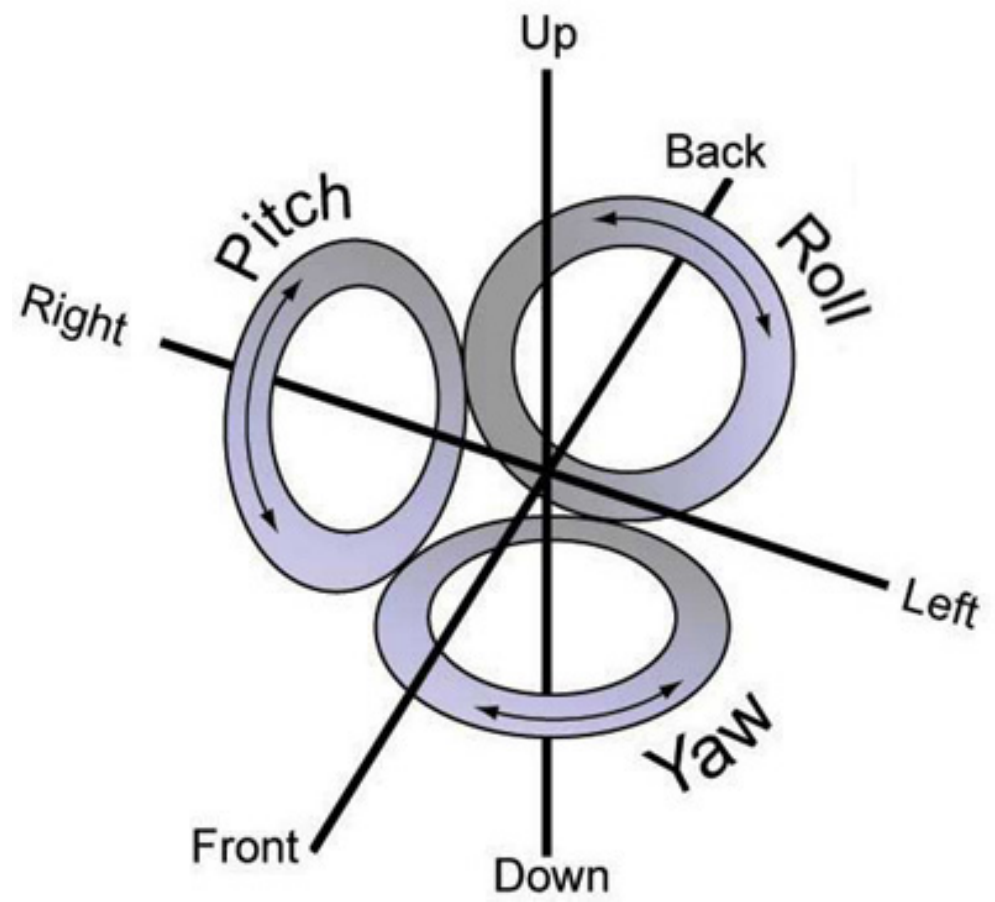

Figure 4 - Yaw, pitch, and roll diagram. Pitch sensations are forward and backward about the right-left axis. Roll sensations are right and left around the front-back axis. Yaw sensations are left and right around the up-down axis [5]. 


\subsection{Types of Stimulation}

Yaw, pitch, and roll sensations are generated through different galvanic vestibular stimulation techniques. Unipolar, bilateral bipolar, and bilateral unipolar stimulation are common configurations for generating sensations [8].

Figure 5shows a sample diagram of the electrode configuration used at the Mayo Clinic and in our experiments to generate orientation sensations. The five electrodes use a combination of unipolar, bilateral bipolar, and bilateral unipolar stimulation to generate the orientation sensations. Each electrode with a number can be individually stimulated in reference to the common (COM) electrode.
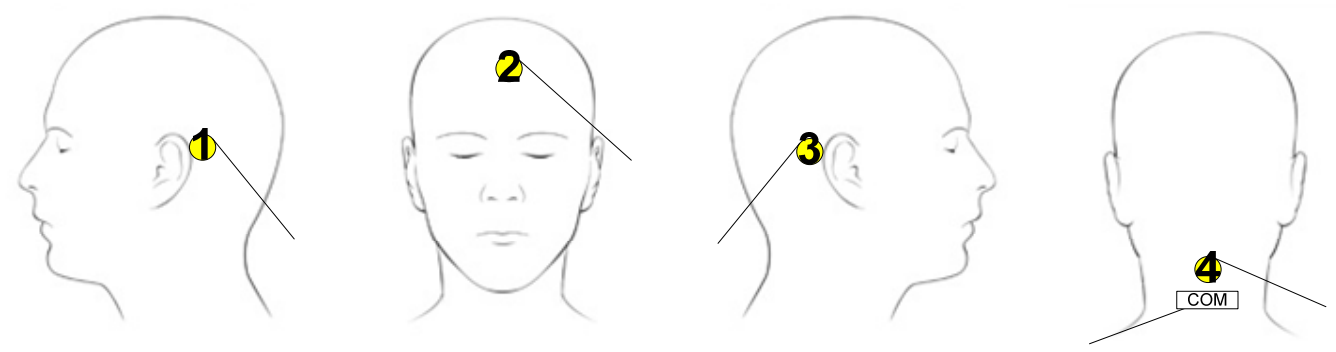

Figure 5 - Electrode placement for sensations of yaw, pitch, and roll. Electrode 1 and Electrode 3 generate the roll sensations. Electrode 2 and Electrode 4 generate pitch sensations.

\subsubsection{Unipolar stimulation}

All of the electrodes in Figure 5 are configured for unipolar stimulation.

Unipolar stimulation consists of an individual anode or cathode electrode

stimulating the body. An example of this would be stimulating a single electrode

(Electrode 1, 2, 3, or 4) in reference to the COM electrode. Although these

unipolar stimulations may generate slight orientation sensations, bilateral bipolar 
and bilateral unipolar electrode combinations are used to generate the orientation sensations.

\subsubsection{Bilateral bipolar stimulation}

Bilateral bipolar stimulation applies current through a human's head by placing a cathode just behind one ear on the mastoid process and an anode behind the other ear on the other mastoid process. The anode is the positive terminal where current enters the body and the cathode is the negative terminal where current leaves the body. This type of galvanic stimulation results in a perceived roll sensation toward the cathode and body sway towards the anode [8]. The sway results from the body trying to compensate for the perceived roll sensation due to stimulation. Additionally, the body lean is dependent on the position of the head. In other words, if the head is rotated so the chin is over the right shoulder and the anode is placed behind the left ear, the body leans forward [6]. Although the perceived roll sensation from bilateral vestibular stimulation is the main sensation, there can also be a small yaw sensation towards the cathode [8]. An example of bilateral bipolar stimulation configuration would be using Electrode 1 as the anode and Electrode 3 as the cathode (as depicted in Figure 5). This configuration would generate a roll right sensation and body sway towards the left.

\subsubsection{Bilateral unipolar stimulation}

A final technique, bilateral unipolar stimulation, places either both cathode or both anode electrodes on the mastoid processes behind the ears. The electrodes behind the ears are referenced to a common electrode placed somewhere else on 
the body such as the neck bilateral unipolar cathode stimulation produces backward sensation and a sway forward of the body [6]. Anodal stimulation produces a forward pitch sensation with a backward sway of the body [8]. An example of bilateral unipolar stimulation would be stimulating both Electrode 1 and Electrode 3 from Figure 5 with anodal current. Bilateral unipolar stimulation in this experiment was only used for the sham signal, explained in section 4.5. The sham signal applies currents large enough for detection but not large enough to elicit a strong pitch sensation.

\subsection{Stimulation into sensations}

Unipolar, bilateral bipolar, and bilateral unipolar stimulations affect afferent firing activity. Adjusting the sensory neuron firing rates of the semicircular canals, macula, and saccula signals to the body a change in orientation has occured [27]. GVS stimulates the semicircular nerves in an unnatural way. The cathode electrode causes all three of the semicircular canals to increase firing activity and the anodal electrode causes all three of the semicircular canals to decrease activity. This section explains in detail how this type of bilateral bipolar stimulation generates an orientation sensation.

Figure 6 shows yaw, pitch, and roll sensations generated through bilateral bipolar stimulation. The markers represent the cristae (crista singular) located inside each ampulla shown in Figure 1. The three semicircular canals are labeled: $a$ (anterior semicircular canal), $p$ (posterior semicircular canal), and $h$ (horizontal semicircular canal). At the base of all the semicircular canals is the utricle represented by a " $u$ ". The left side of Figure 6 represents anodal stimulation or the 
positive terminal of the battery connected to the right side of the body. The right side of the figure represents cathode current or the negative terminal of a battery connected to the left side of the body. For yaw, pitch, and roll Figure 6 shows the corresponding direction of the virtual sensation generated from stimulating each semicircular canals. It should be noted that the brain uses information from both semicircular canals to interpret orientation information. In normal operation both canals provide consistent sensory information. Since GVS stimulates all of the canals, the brain sometimes receives conflicting data which does not result in an orientation sensation.

Anodal stimulation on the right side of the body and cathode stimulation on the left side results in a virtual sensation towards the cathode and a body sway towards the anode. Both of the horizontal canals cause yaw towards the cathode or left side of the body. The anterior and posterior canals roll to the left or towards the cathode as well. On the right side of the body the anterior canal causes backward pitch, while the posterior causes forward pitch. The anterior and posterior pitch sensations are reversed for the left side of the body. These opposing pitch sensations cancel each other out and result in no pitch sensation. In summary the anodal stimulation on the right side of the body and cathode stimulation on the left causes the same yaw and roll sensations towards the cathode, but pitch sensations in opposing directions [8]. 


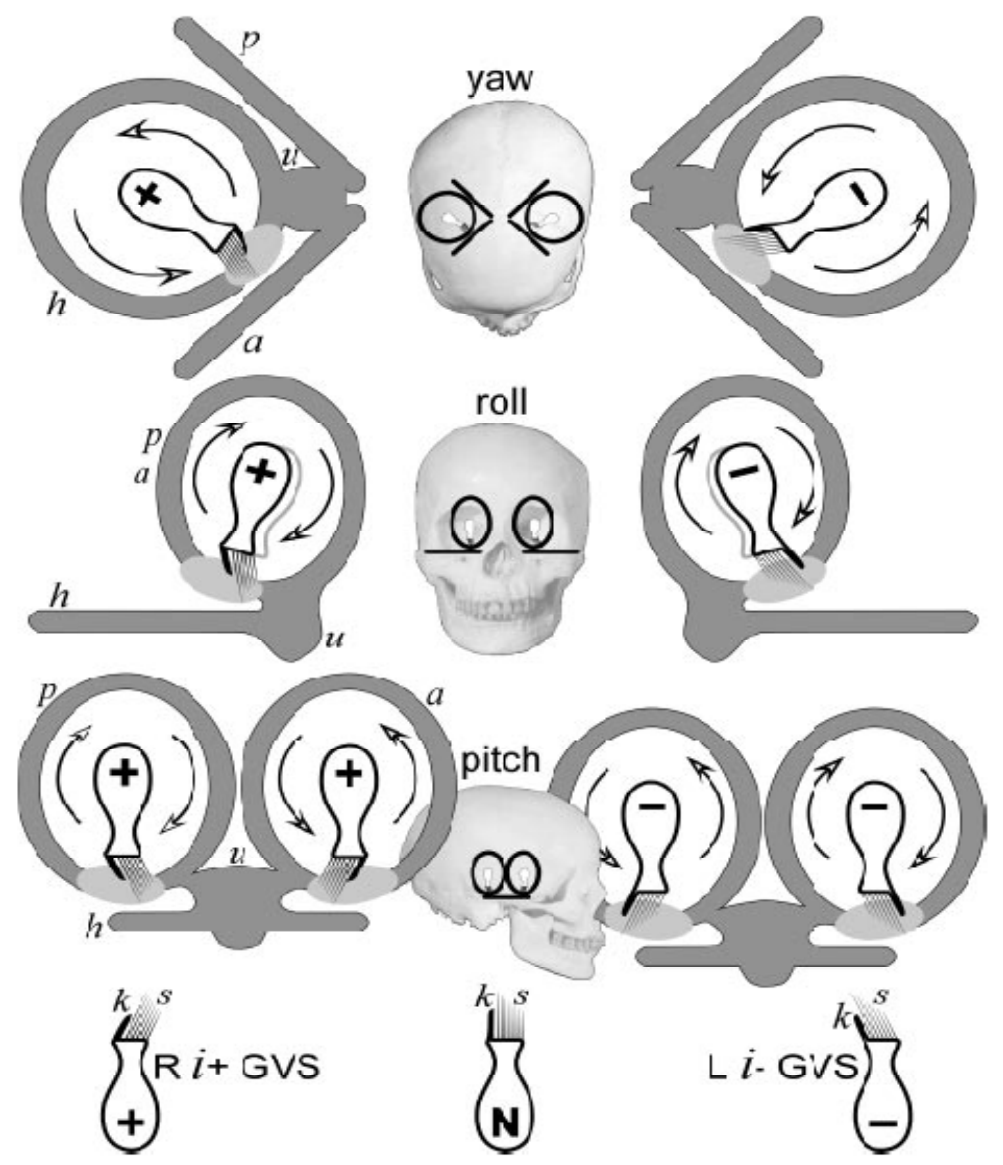

Figure 6 - Fitzpatrick's yaw, pitch, and roll sensations from bilateral bipolar vestibular stimulations. With the anode connected to the right side of the body and the cathode connected to the left side of the body, the stimulation generates slight yaw towards the left side of the body, roll sensation towards the left side of body, and no pitch sensations [8].

The utricular and saccular maculas are also stimulated as a result of the currents induced by the electrodes. As noted earlier, each macula has two regions of opposing hair cell polarities. The GVS stimulations cause conflicting sensory information from the two regions. If the maculae were the exact same size there would be no sensation. Since they are not the same size slight sensations are generated from the macula [8]. However, most of the sensations are generated from the semicircular canals. 


\section{CHAPTER 2 : Electricity and the Human Body}

\subsection{Electrical properties}

The body's response to current entering the body depends on the electrical properties of that specific person. "The electrical properties of skin can be changed by a variety of conditions, including degree of hydration, presence of chemical additives, electrolyte concentration and valence, temperature, time of year, perspiration, skin disease, thyroid activity, and emotional state" [20].

Properties of a current stimulation such as waveform and duration also contribute to the electrical properties of the body [20]. Figure 7 and Figure 8 show the impedance magnitudes and impedance phases as functions of frequency respectively.

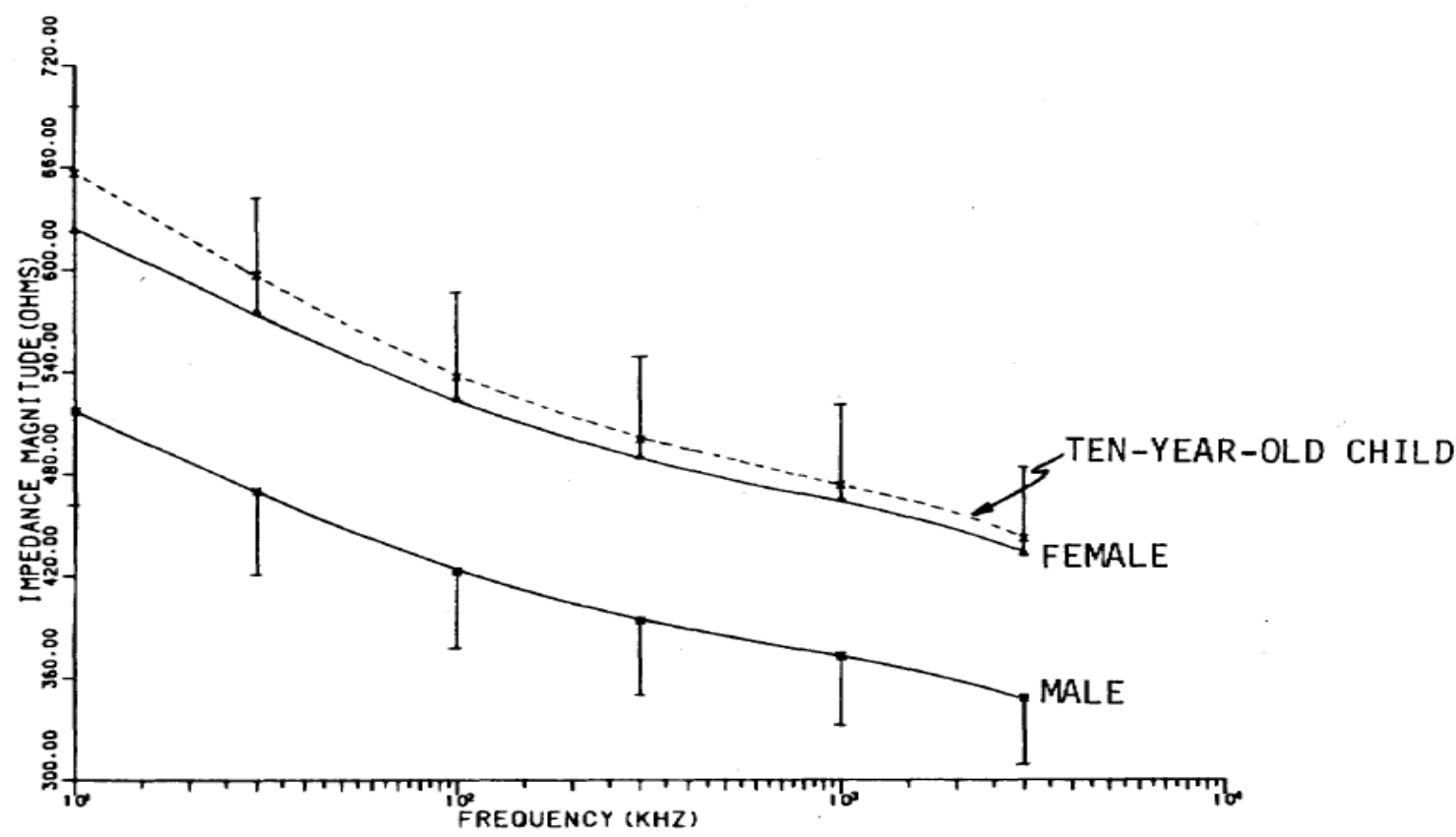

Figure 7 - Impedance Magnitude as a function of frequency. The plots are for a male, female, and a ten-year-old child as noted on the plot [4]. 


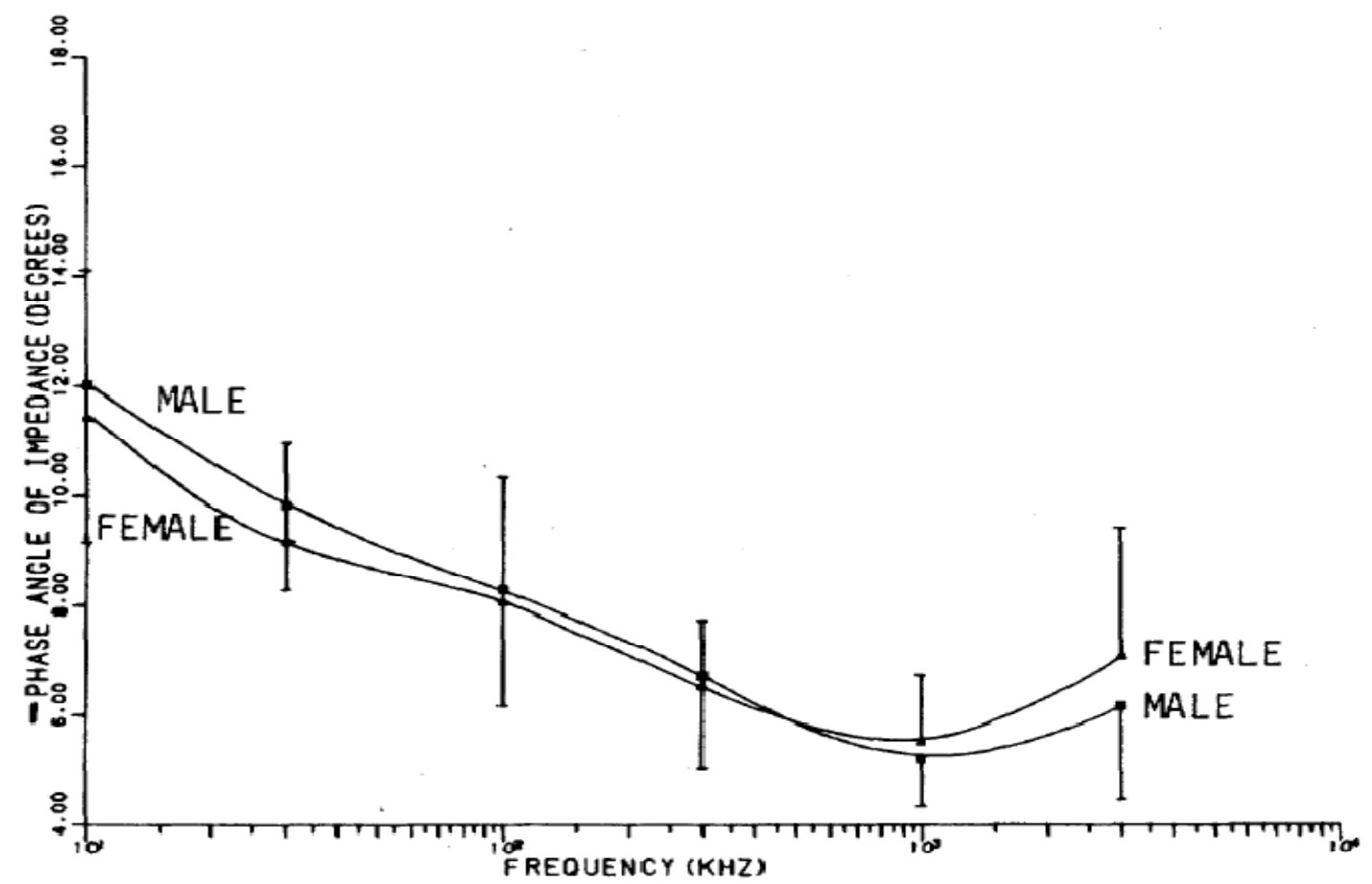

Figure 8 - Impedance Phase as a function of frequency for a female and a male as noted on the plot [4].

The human impedance measurements were taken from the right index finger to a ground plate connected beneath both bare feet of the subject [4]. The plots indicate that as frequency goes up the magnitude of the impedance and the phase angle tend to go down.

Figure 9 is a simple example of an equivalent circuit for modeling the electrical properties of the human body with linear and nonlinear circuit elements [20]. $\mathrm{R}_{\mathrm{sc}}$ and $\mathrm{C}_{\mathrm{sc}}$ represent properties of the outer skin layer, the stratum corneum. This layer of skin typically has resistances $\mathrm{R}_{\mathrm{sc}}$ in the range of $10^{4}-10^{6}$ $\Omega \mathrm{cm}^{2}$ and capacitances $\mathrm{C}_{\mathrm{sc}}$ in the range of $1-50 \mathrm{nF} / \mathrm{cm}^{2}$. Resistor $\mathrm{R}_{\mathrm{s}}$ represents the inner tissues of the body and is typically in the range of $100-200 \Omega \mathrm{cm}^{2}$. The 
impedance on the outer layer of the skin is much higher than the inner layers. As shown by the units, electrical properties of the skin have been shown to be proportional to area [20].

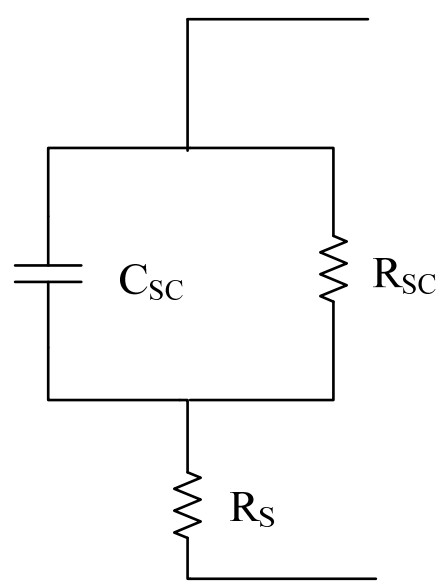

Figure 9 - Equivalent circuit model for electrical properties of the skin. The $\mathbf{C}_{\mathrm{SC}}$ and $\mathbf{R}_{\mathrm{SC}}$ represent the strum corneum outer layer of the skin. Rs represents the inner tissues.

When stimulating a human there are a few reasons it is important to have insight into the electrical properties of the body. The first reason is that it is important to get the impedance of the body low enough for the stimulations to be effective. Impedance measurements can be affected by factors such as the amount of dead skin cells and dirt on the surface of the skin. The large and highly variable impedance of the body acts as a protective layer against shock and subsequent stimulations. In addition, electrical properties contribute to the current threshold, the pain threshold, muscle stimulation, and overall safety of the subject. 


\subsection{Electrical sensations}

In order to safely and effectively administer the galvanic stimulations it is not only important to know about the electrical properties of the body, but it is important to know what the subject feels as a result of those properties. Current entering the body has a possibility of two sensations [19]. The first sensation that can be felt is known as the "threshold of perception." This is the point at which a human is aware current is entering the body. The "threshold of perception" happens when the current is large enough to fire nerves at the stimulation site. This threshold varies just as the impedance magnitudes and the phase magnitudes varied in Figure 7 and Figure 8. Figure 10 shows the threshold current as a function of frequency for measurements take from the right index finger to a ground plate underneath bare feet. The plot indicates that the "threshold of perception" for a $10 \mathrm{KHz}$ signal is typically around $3 \mathrm{~mA}$. For signals less than 10 $\mathrm{KHz}$ such as DC currents, the "threshold of perception is around $2 \mathrm{~mA}$ from finger to finger [19]. With the electrodes connected to the mastoid process, the subjects in our testing had a lower threshold. The "threshold of perception" in our subjects typically happens around $0.4 \mathrm{~mA}$. For the vestibular stimulations, it is desired to minimize the "threshold of perception" while maximizing the orientation. 


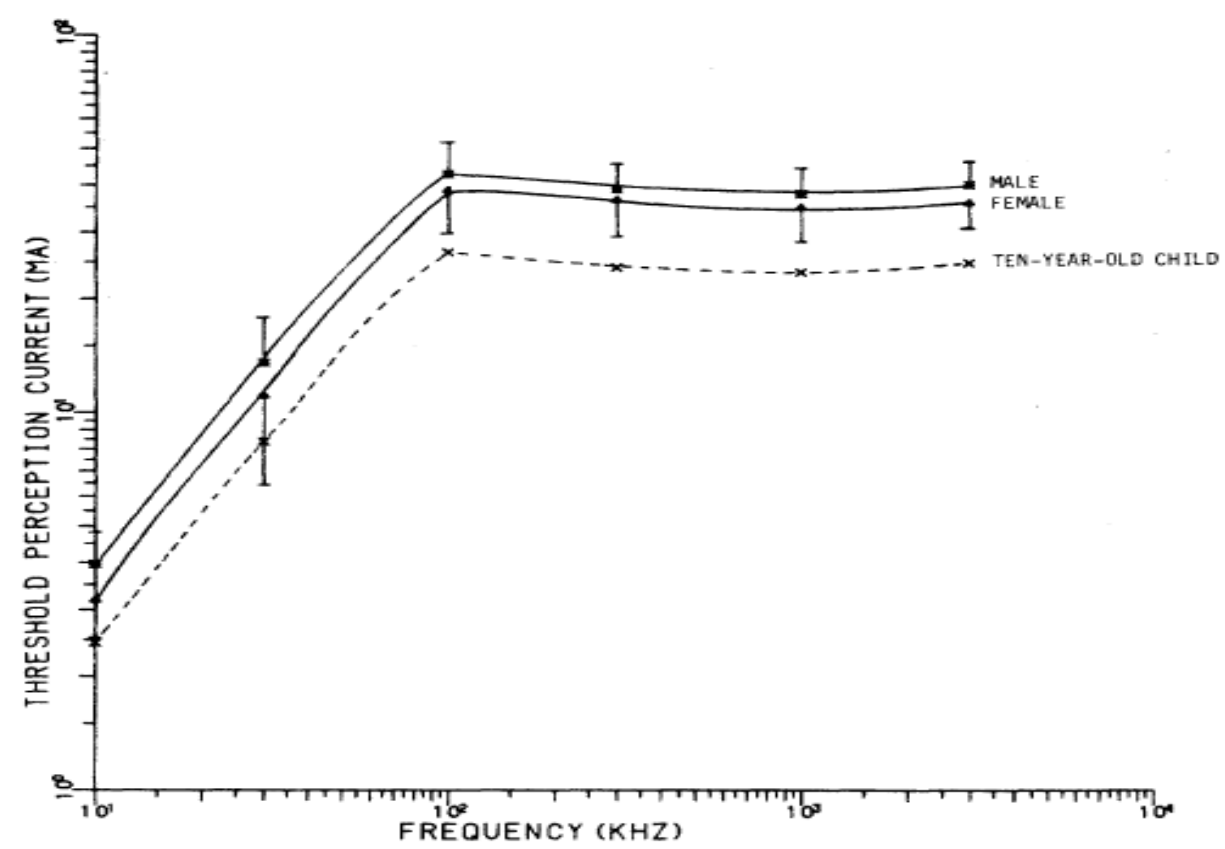

Figure 10 - "Threshold of Perception" as a function of frequency for 10 year old child, female, and male [4].

As current continues to increase beyond the "threshold of perception", it approaches the second sensation, the "threshold of pain." "Pain thresholds, which identify the minimum current applied to the skin which causes a painful sensation, are more variable than perception thresholds, as they depend on both physical and sociological factors" [20]. Figure 11 shows the pain threshold as a function of frequency. In our experiment, the current is ramped up prior to beginning the tests to ensure stimulations are below the "threshold of pain". 


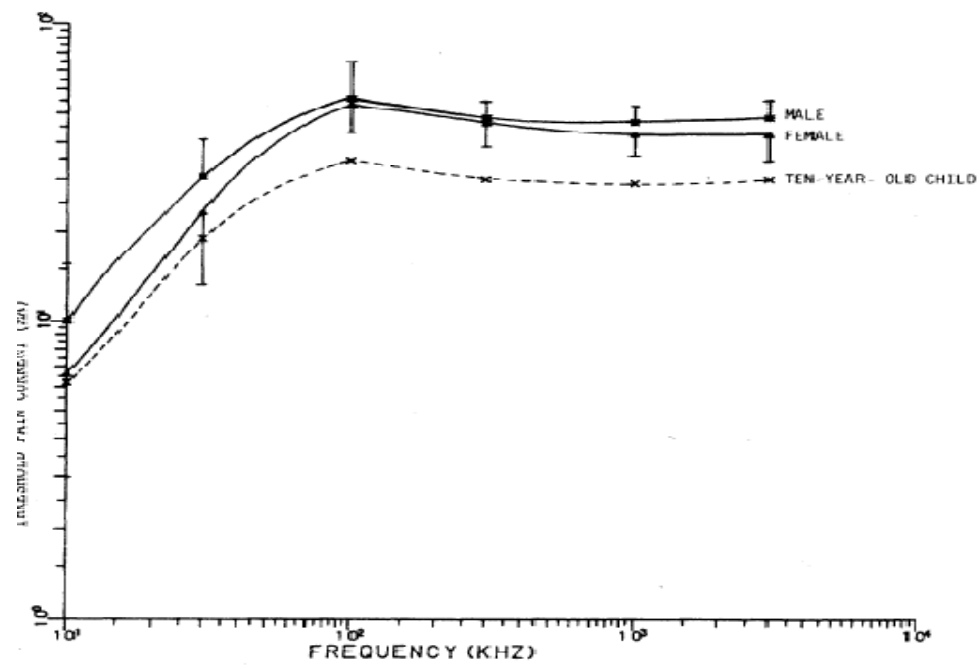

Figure 11 - Threshold of Pain as a function of frequency for a ten-year- old child, female, and male [4].

\subsection{Muscle contractions}

The stimulation of muscles can occur directly from the electrical stimulation of muscle cells or indirectly from the stimulation of nerves which trigger a muscle contraction [20]. Again, the levels of current necessary to stimulate a muscle directly and indirectly vary with electrical properties. However, direct stimulation of a muscle typically happens at currents 2-3 orders of magnitude larger than indirect muscle stimulation [20]. Most medical applications stimulate muscles by a sequence of pulses. In our experiments it is not desired to stimulate muscles. Ear and skin twitches resulting from stimulation provide distracting sensory information not related to orientation. If muscle stimulations are too high the maximum stimulation current level is lowered to try to reduce or eliminate the muscle contraction. Sometimes the muscle stimulation can be prevented by rapidly reversing the current after the stimulating pulse [20]. However, the stimulations in our experiments are too long to use this approach. 


\subsection{Electrical safety}

The final issue related to electrical properties of skin is electrical safety. What are the safety issues and how are they prevented? Figure 12 graphs various human thresholds and sensations as a function of current for a $60 \mathrm{~Hz}$ sine wave stimulating from one human hand to the other. The plot outlines general safety considerations when stimulating the human body.

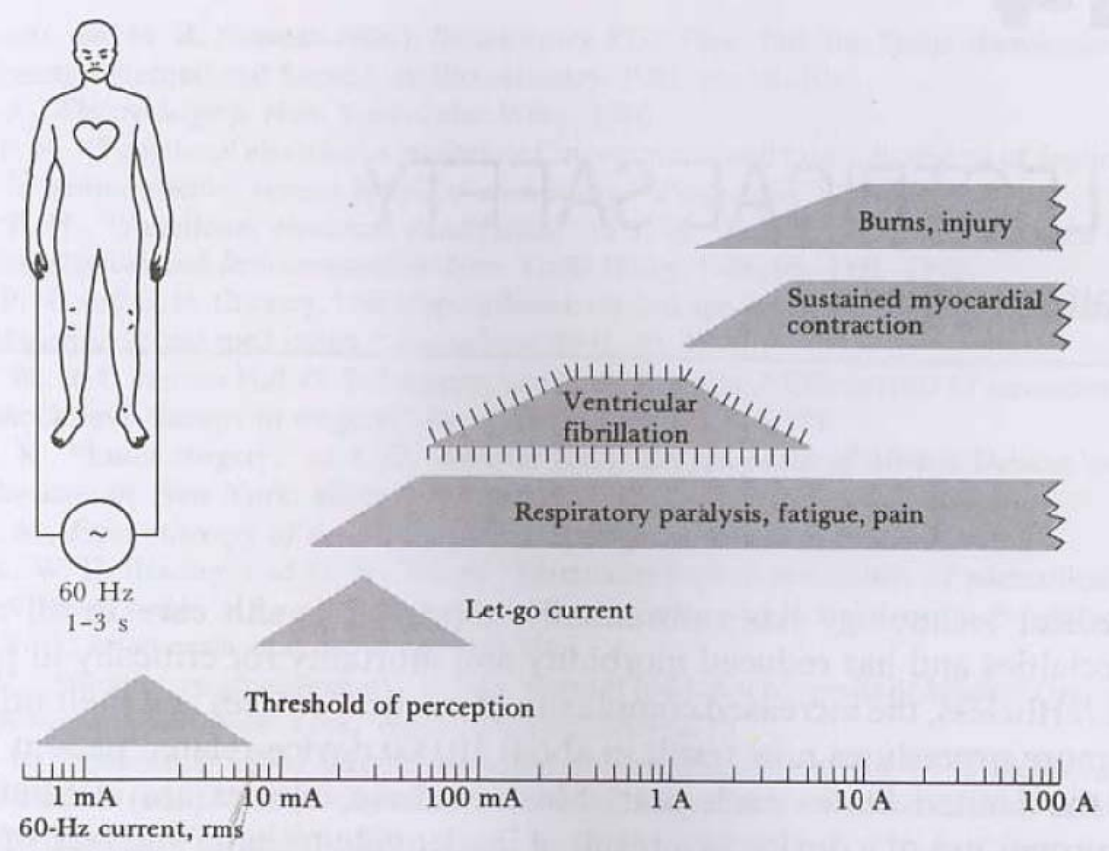

Figure 12 - Electrical Safety current thresholds for a $60 \mathrm{~Hz}$ sin wave. Thresholds are for electrodes connected from one hand to the other [19].

Figure 12 shows safety thresholds including "threshold of perception", "let-go current", respiratory paralysis and pain, ventricular fibrillation, myocardial contraction, and burning. In the discussion of electrical safety of GVS, many of these same safety thresholds apply. Improper stimulation of the body can cause resistive heating, skin irritation, nerve damage, and heart problems. The effects of 
these safety hazards range from minor discomfort to possible death. Each of these safety concerns are addressed in relationship to the experiments conducted.

\subsubsection{Resistive heating}

In Figure 9 the human body was modeled as a resistive-capacitive circuit. With this model the body has both internal and external resistances dissipating heat. It is possible for the dissipating heat to cause cells to get too hot. The outer layers of the skin, the stratum corneum, have a much larger resistance, typically dissipating most of the heat. However, high voltages change the electrical properties of the skin and can cause resistive heating throughout the body [20]. Typically $45^{\circ}$ Celsius or $113^{\circ}$ Fahrenheit is accepted as the safety limit for skin heating to prevent burning [20]. This experiment does not use high voltages. The maximum voltage level possible by the battery source is 12 volts. The maximum current the device can output is $2.65 \mathrm{~mA}$. These voltage and current levels are far below those needed to heat the skin to damaging levels.

\subsubsection{Skin irritation}

Current entering the body can not only cause resistive heating and burns, but can also cause skin irritation. The main cause of the skin irritation results from changes in $\mathrm{pH}$. When current enters the body the skin undergoes electrochemical changes and increased ion transport resulting in a change in $\mathrm{pH}$ [20]. The current stimulation alters the polarity of the nerve to either increase or decrease firing activity. The change in $\mathrm{pH}$ of the neurons is natural. The easiest way to mitigate the $\mathrm{pH}$ changes at the surface of the skin is to use $\mathrm{Ag} / \mathrm{AgCl}$ electrodes. These 
electrodes prevent $\mathrm{pH}$ changes by hydrolysis [20]. In this experiment disposable $\mathrm{Ag} / \mathrm{AgCl}$ electrodes are used for the stimulations.

\subsubsection{The heart}

One of the largest concerns with stimulating the body is the human heart. The leading cause of death with shocks or stimulations under 300 volts is cardiac ventricular fibrillation [20]. Ventricular fibrillation can cause a heart attack and instant death. Typical thresholds for humans range from about 75 to $400 \mathrm{~mA}$ [19]. There are two strategies to avoiding ventricular fibrillation of the heart during stimulation. The first obvious strategy is to avoid reaching currents high enough to result in ventricular fibrillation. As noted earlier the maximum current used in the tests is $1.5 \mathrm{~mA}$. The second strategy is to avoid electrode placement that cause the heart to be in the main path of the current. For example, don't put an electrode on the sternum and another electrode on the middle of the back for stimulations. As Figure 5 hows, all of the electrodes in this experiment are placed on the head.

\subsubsection{Long term exposure}

Another issue associated with electrical safety results from the duration of stimulation. Prolonged stimulation of up to 2000 hours can cause nerve damage. "At greater charge densities damage was seen as increased bone growth and cell death" [20]. Since this experiment is stimulating nerves, it is important to do so in a manner that does not result in nerve damage. In our experiments, the longest the electrodes are physically secured to a subject is approximately 90 minutes. The longest a subject receives stimulations is approximately 35 minutes, and during that time the electrodes are not continuously stimulating the subject. The 
stimulation lengths are much shorter than 2000 hours. The flight tasks are designed so that if the first task is done correctly there is not any stimulation. If the second task is completed, there should be about four stimulations for each of the three different stimulation profiles. The stimulations last as long as the subject is banked past $+/-30$ degrees. The final chase plane task has the most stimulation, but with all three stimulation profiles the entire chase plane task does not exceed 15 minutes. 


\section{CHAPTER 3 : GVS Literature Review}

Otolithic and semicircular canal responses result from vestibular stimulation at low current intensities of 0.5-3 mA, resulting in direction specific sway [27]. However, the galvanic vestibular response is complex and modifiable by many factors including: the availability of non-vestibular sensory information, standing posture, head and trunk position, and support surface properties [13]. This chapter discusses these issues and their application to this project.

\subsection{Ocular effects of GVS}

Visual cues and vestibular sensory information are used for determining orientation. Guerraz and Day make a distinction of visual sensations indicating "external motion" and vestibular sensations detecting "self motion" [14]. This distinction is key to understanding how the body interprets orientation information from the two sensory inputs.

The authors designed an experiment to test the effects of expectation on vestibular stimulations. In their study, they indicate that the "visual channel of balance control is susceptible to cognitive influence" [14]. If the body anticipates motion as a result of the outside world, it suppresses orientation sensory information. They suggest that the reason the body suppresses responses resulting from visual channels and not the vestibular system is because the vestibular system always signals self motion. On the other hand, "Visual flow is ambiguous in that it signals object motion, eye motion, as well as self-motion" [13].

GVS causes visual and vestibular sensations. Whether the subject under test is in a light or dark room, expects or does not expect the stimulation, the result is the 
same [14]. When current intensities exceed $3 \mathrm{~mA}$, vestibular stimulation causes binocular torsion of 0.5-5.0 degrees toward the anode in addition to a perceived room tilt of 1.0-9.4 degrees in the opposite direction [27].

\subsection{Postural Responses to GVS}

Studies have shown that many postural muscles in the neck, back, legs, and ankle are activated during GVS, such as the calf or soleus muscle [24]. An experiment by Day et al. measured the amount of tilt in standing subjects in various foot positions as well as sitting postures. The orientation sensations and relative tilts are much smaller when seated than when standing [6][24]. When standing the body tilt increased as the width in stance decreased. This result indicates that the response to GVS is organized to stabilize the entire body rather than just the head [6].

A common task affected by stimulating standing subjects is walking. In an experiment by Bent et al. the walking trajectory of stimulated humans was investigated. All of the walking subjects swayed towards the anode. For each subject a threshold current was determined when the sensations became apparent to that subject. The walking subject was then stimulated at one, two, and three times the threshold. Figure 13 shows a representative average of one subject's walking path with each of the stimulations. Stronger current stimulations result in larger walking deviation. 

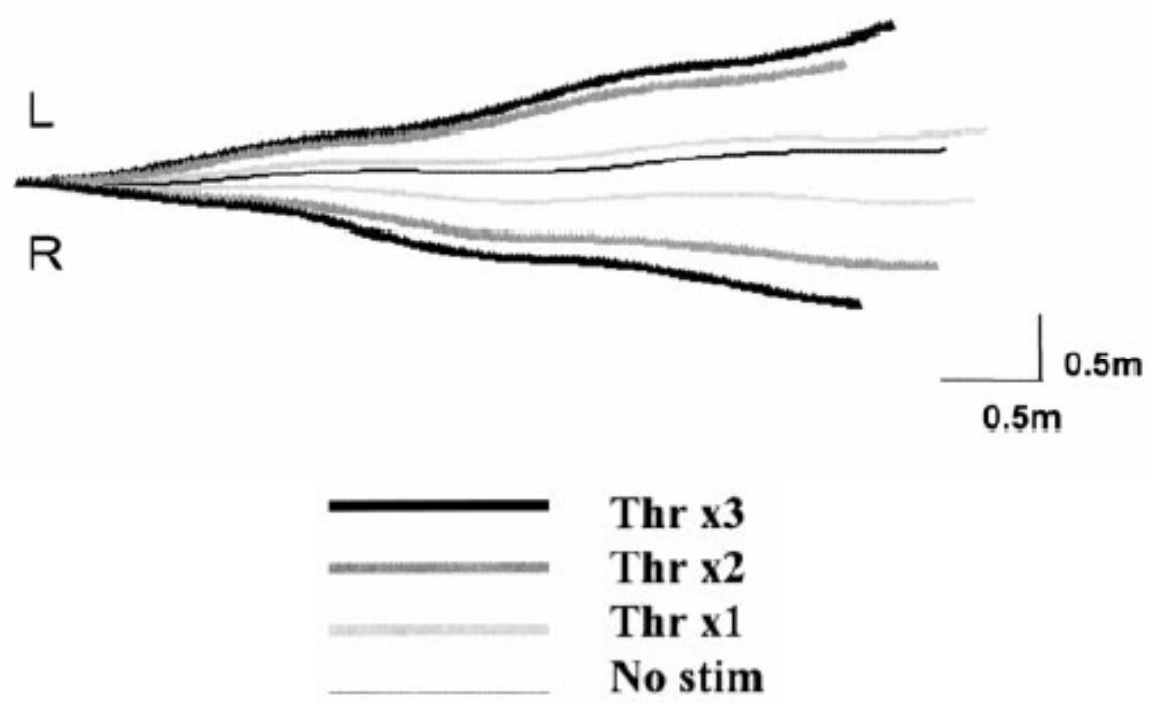

Figure 13 -Walking trajectory vestibular stimulation data. X1 shows the walking trajectory at stimulations of 1 times the threshold current. $X 2$ was double the threshold, and X3 was triple the threshold current. The trajectory paths increased with current level [1].

In another experiment by Scinicarello et al, GVS was used in standing subjects to enhance balance control. The subjects stood on a platform that perturbed horizontally; GVS was used to mitigate the postural sway effects of the platform. The natural balance mechanism of the body was compared with the GVS balance mechanism. The GVS postural correction was quicker and more accurate than the natural balance system of the body [21]. This example has many potential applications in minimizing disorientation and vestibular disorders. However, it is ambiguous if these corrections were hindering the vestibular system's ability to detect information regarding changes in the platform or if the stimulations resulted in an actual override of information sent to the brain.

\subsection{Brain Response}

Another study investigated the activated areas of the brain in six right-handed males during GVS with the use of Functional Magnetic Resonance Imaging 
(fMRI). Figure 14 shows the seven areas of increased activation resulting from the stimulations. There was also one area of activation that decreased activity.
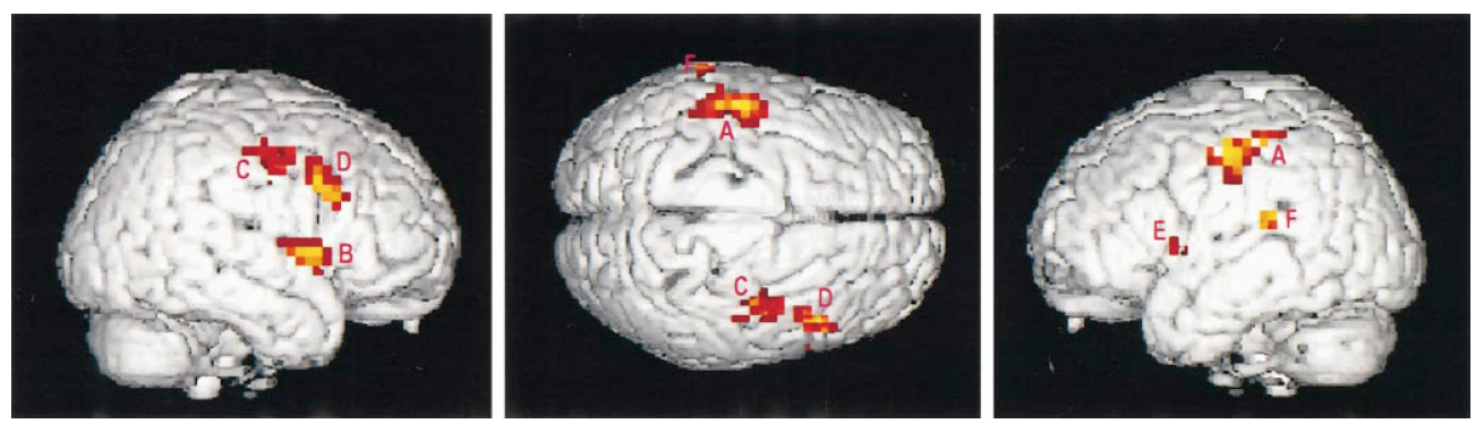

Figure 14 - Areas of activation in the right, superior, and left hemispheres of the brain. There were seven main areas of activation and one area of decreased activity [17].

The important result from this study was that all of the subjects had the same areas of the brain activated. However, within each of these areas there was variability. There are three factors that may be contributing to the intervariablity within the activated regions. The first reason is that the brain must be stationary during stimulation, so any movement of the head adjusts the area being scanned [17]. The second factor is the variable number of Type I and Type II neurons in the corticovestibular regions of the brain. The increase or decrease in neuron type activation varies from person to person despite the same vestibular stimulus. The third possibility is varying galvanic sensitivity to vestibular afferents. The study confirms that the stimulations are activating the same regions of the brain.

However, there may be some differences in the information transmitted to the brain as a result of the stimulation, and some variability in the interpretation of those sensations [17]. 


\subsection{Perceived sensations response time}

A study by Barnett-Cowan et al. explored the perceived response time of a number of modalities. Figure 15 shows response times to GVS, touch, light, and sound stimulations. All of the stimulation response times were estimated by having the subject press a button upon stimulation. The response time for the GVS stimulation was $250 \mathrm{~ms}$ longer than the detection time of light or sound. According to those detection times, the vestibular sensation must happen prior to the visual sensation in order for an event to be detected simultaneously. It is possible that the brain compensates for varying times in stimulation detection [2].

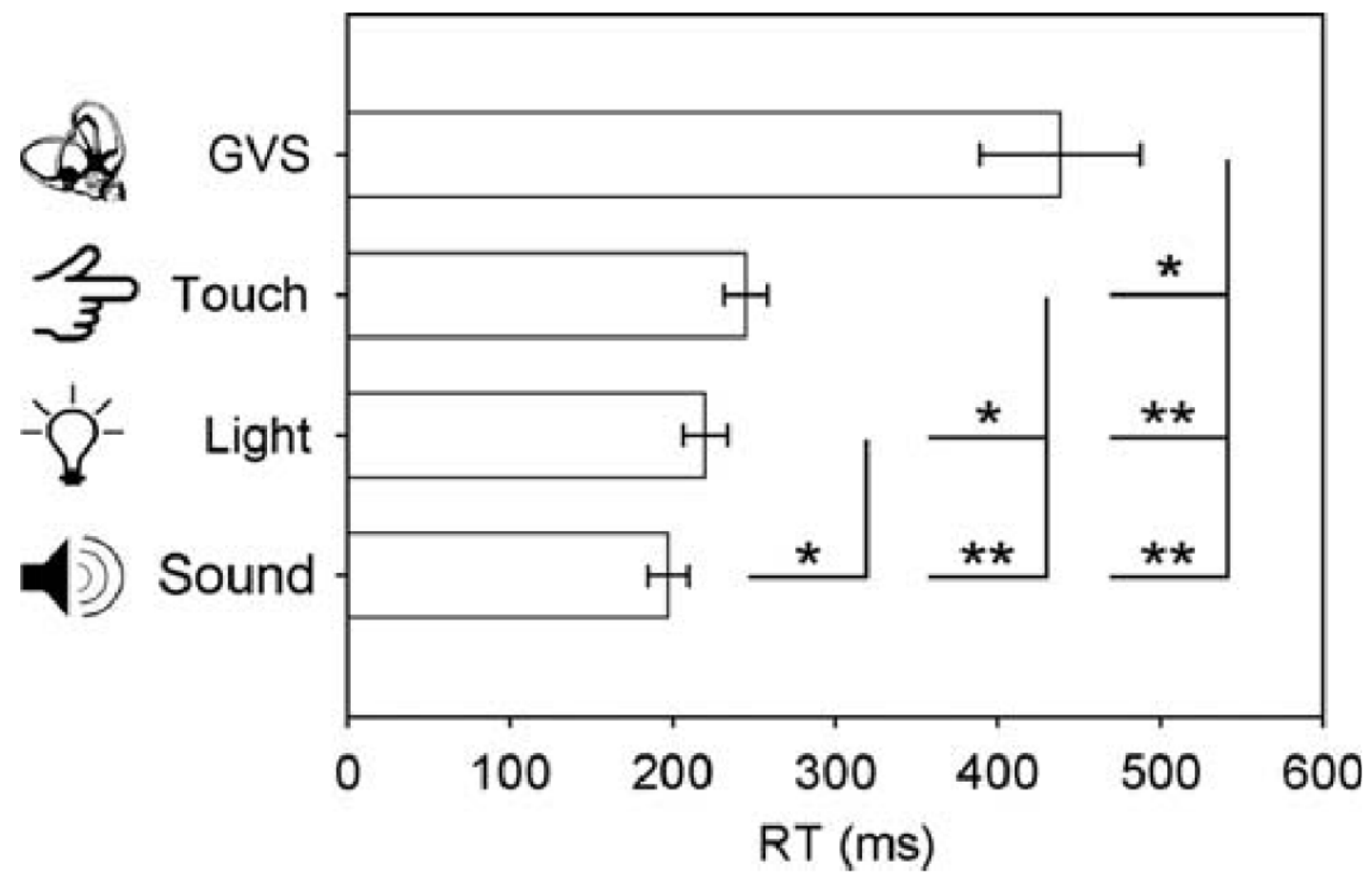

Figure 15 - Response time to GVS, touch, light, and sound stimulations [2] 


\subsection{Relation between literature review and our work}

The ocular effects, the postural responses, the brain response, and the perceived sensations response time studies all provide insight into the design and implementation of GVS applied in our motion sensitivity and flight simulator experiments. The specific experimental protocols and equipment used in each of these tests are described in Chapters 4 and 5.

\subsubsection{Ocular effects}

Guerraz and Day described binocular torsion and perceived room tilt as a response to "self motion", which is not influenced by cognitive interpretation. In other words, both the binocular torsion and the perceived room tilt are repeatable vestibular responses indicating to the body a change in orientation has happened. In both our experiments it is desired to have repeatable vestibular responses to the galvanic stimulations, however the forced binocular torsion may impair a subject's ability to focus on a visual in the flight simulator experiment.

\subsubsection{The postural Response}

The Day et al. study expands on the notion of repeatable vestibular responses through testing seated versus standing subjects. GVS provides stronger sensations in standing subjects. The, Bent et al. study, provided additional insight demonstrating that increasing the current level increases the response of the GVS. In the motion sensitivity it is important to maximize the orientation sensations generated during GVS. Therefore, the subjects receive the test standing and ideally the stimulations use the highest safe current amperage. In the flight simulator tests the subjects remain seated in order to simulate a more realistic 
flight environment. The dosed current response can be used to program the flight simulator.

\subsubsection{The brain responses}

Studies related to the ocular and the postural responses promote GVS as a means to consistently generate a sensation to the body that a change in orientation has occurred. Lobel et al used function MRI scans to record areas of the brain activated during galvanic stimulations. The seven main areas of activation and one area of decreased activity resulting from the stimulations supports GVS as a repeatable common response to the stimulations. However, within common areas of activation there was some variability. In both the motion sensitivity test and the flight simulator test it is important to have repeatable stimulations, and although the article presents possible causes, it should be noted that some variability in the responses may exist.

\subsubsection{Perceived sensation response time}

Barnett-Cowan et al. noted differences in perceived response time across multiple modalities, including differences between light, sound and GVS. The time difference is worth noting for our study, which pairs GVS with a flight simulator to create a more realistic virtual flight environment. 


\section{CHAPTER 4 : System Design}

There are two primary experiments which were conducted. The first experiment was conducted to evaluate GVS as a predictor for susceptibility to motion sickness. Figure 16 shows the basic system design used for performing this test, which consists of a subject, the GVS device, and custom software.

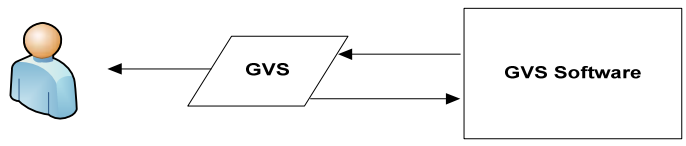

Figure 16 - System block diagram for the motion sensitivity test consisting of the GVS Device and the GVS Software

The second experiment aimed to explore the effects of GVS stimulation coupled with coupled with a visual stimulus on the vestibular system. In this experiment X-Plane, a Plane, a flight simulator (www.x-plane.com), provided the visual stimulus. The system design for this experiment included additional computers to run the $\mathrm{X}$ X-Plane simulator (

Figure 17).

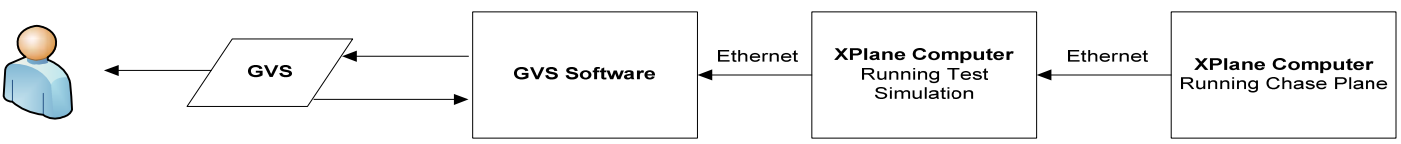

Figure 17 - System block diagram for the X-Plane Virtual Flight Simulator including the GVS, the GVS software, a computer running X-Plane controlled by the subject, and a computer running the $X$-Plane chase plane.

\subsection{The human body}

The subject's body is the first block in the system design. The subject is connected to the GVS device through electrode 1 and electrode 3 from Figure 5 which generates the roll sensations. Electrode 2 and electrode 4 were not included 
in this experiment due to a lack of pitch sensations generated with our setup. In order to ensure intended current levels, the subject's impedance must be low enough for the 12 volt battery source to supply the maximum intended current levels. In order to reduce the impedance of the outer layers of the skin, a prep procedure is conducted, including application of a skin prep cream explained in more detail in Chapter 5 .

The maximum current used during testing is $1.5 \mathrm{~mA}$. By Ohm's law with the 12 volt battery source, the ideal maximum allowable resistance for these tests should not exceed $8 \mathrm{k} \Omega$. However, the maximum allowable resistance used for these experiments' was $10 \mathrm{k} \Omega$. This value was selected due to variability in the resistance measurement. The clearly non-resistive properties of the skin shown in the impedance plots from section 2.1 indicate the skin behaves nonlinearly. Therefore the resistance measurement is not constant. An RLC Meter would provide more accurate information into the electrical properties of each subject's body, but the intention was to take a quick reference measurement.

There was also variability in the allowable current levels by the subjects. If the subject would not allow the full $1.5 \mathrm{~mA}$ the allowable resistance would increase to above $8 \mathrm{~K} \Omega$. In addition the resistance of the body goes down as the electrodes sit on the skin and as current flows into the body. The most important thing is that the subject receives enough current to stimulate the vestibular system. 


\subsection{Galvanic Vestibular Stimulator (GVS) Device}

The GVS device in Figure 18(a) is manufactured by and was purchased from Good Vibrations Engineering in Toronto, Canada. The device comes with 4 independent current sources. Each electrode source can output current in the range of $2.5 \mathrm{~mA}$ to $-2.5 \mathrm{~mA}$ at increments of $0.0195 \mathrm{~mA}$. The GVS is powered by $8 \mathrm{AA}$ batteries. Figure 18 provides a top level block diagram of the GVS device. Figure 18(b) provides a top level block diagram of the GVS device.

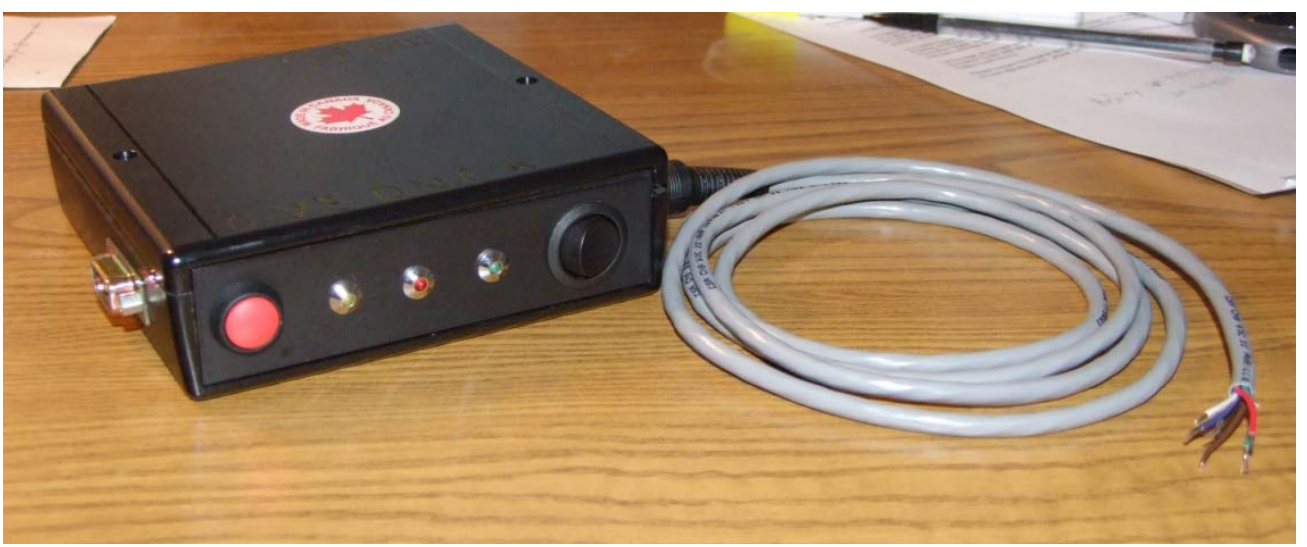

(a) External GVS Device

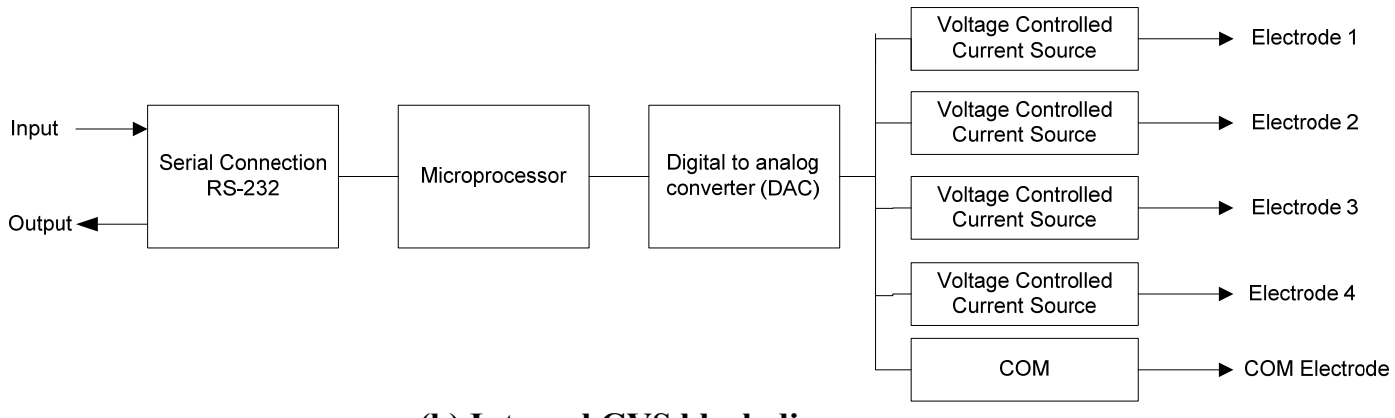

(b) Internal GVS block diagram

Figure 18 - GVS Device (a) External GVS Device (b) Internal GVS block diagram 


\subsubsection{RS-232 Interface}

The first stage of the device consists of an RS-232 serial connection to communicate between an external device (such as a PC issuing commands to the GVS device) and the microprocessor. The RS-232 connection works with either Bluetooth or a serial cable. A serial cable is faster than the Bluetooth, but the cable must be a null-modem cable, which allows each device to transmit received and sent information. As done in this experiment, it is possible to create your own cable from a standard serial connector by switching the wire 2 (red) and wire 3 (orange) on one side of the connector. The only three wires necessary for communication for the GVS device are wires 2, 3, and 5 (green) of a serial connector.

\subsubsection{Microprocessor Messages and Commands}

The microprocessor receives the commands from a computer via the serial port and sends messages back to the computer in response to the commands. It takes a minimum of five bytes to send a message or a command; commands and messages sent both have the same format. Figure 19 summarizes the command/message protocol in a sample byte stream.

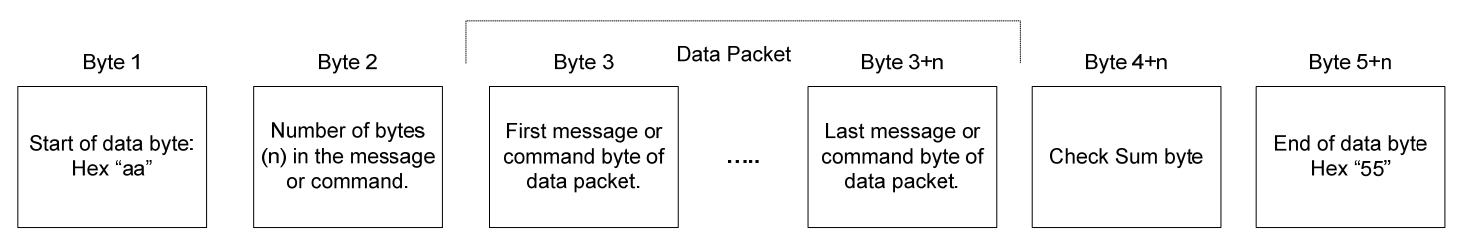

Figure 19 - Sample byte stream for message or command transmission.

The first byte is a "start of command" or "start of message" initializer. The initializer has a hexadecimal value $0 \mathrm{Haa}$. The second byte indicates the number of 
messages or commands included in the following data packet. If there was only one message byte in the packet then this value would equal one. The $n$ value represents the number of bytes in the message or command data packet in addition to the first byte. For example, if there were a total of 3 bytes in the packet " $n$ " would equal 2. Again, the byte prior to the data packet indicates the total number of bytes in the data packet, so it is equal to $n+1$. The next byte or group of bytes represents the actual commands/messages is called the data packet. The byte following the data packet is the checksum value, which is used for detecting errors in data transmission. The checksum byte adds the value of each data byte. The final byte in the command is the "end of message" or "end of command" byte, $0 \mathrm{H} 55$.

\subsubsection{Digital Signal into Electrical Stimulation}

The commands sent from the computer are processed by the microprocessor. If a command was sent for a change in electrode current or a change in all the electrode currents, the microprocessor outputs a corresponding digital signal to the digital to analog converter (DAC). The DAC output voltage drives the voltage controlled current source, which stimulates the vestibular system of the subject. Figure 20 shows the typical application from the datasheet of the BB INA105KP precision unity gain differential amplifier chip used in this GVS device. Each of the electrodes uses this chip as a voltage controlled current source. The software in the microprocessor limits the device from outputting more than 2.54 milliamps or less than -2.56 milliamps. 


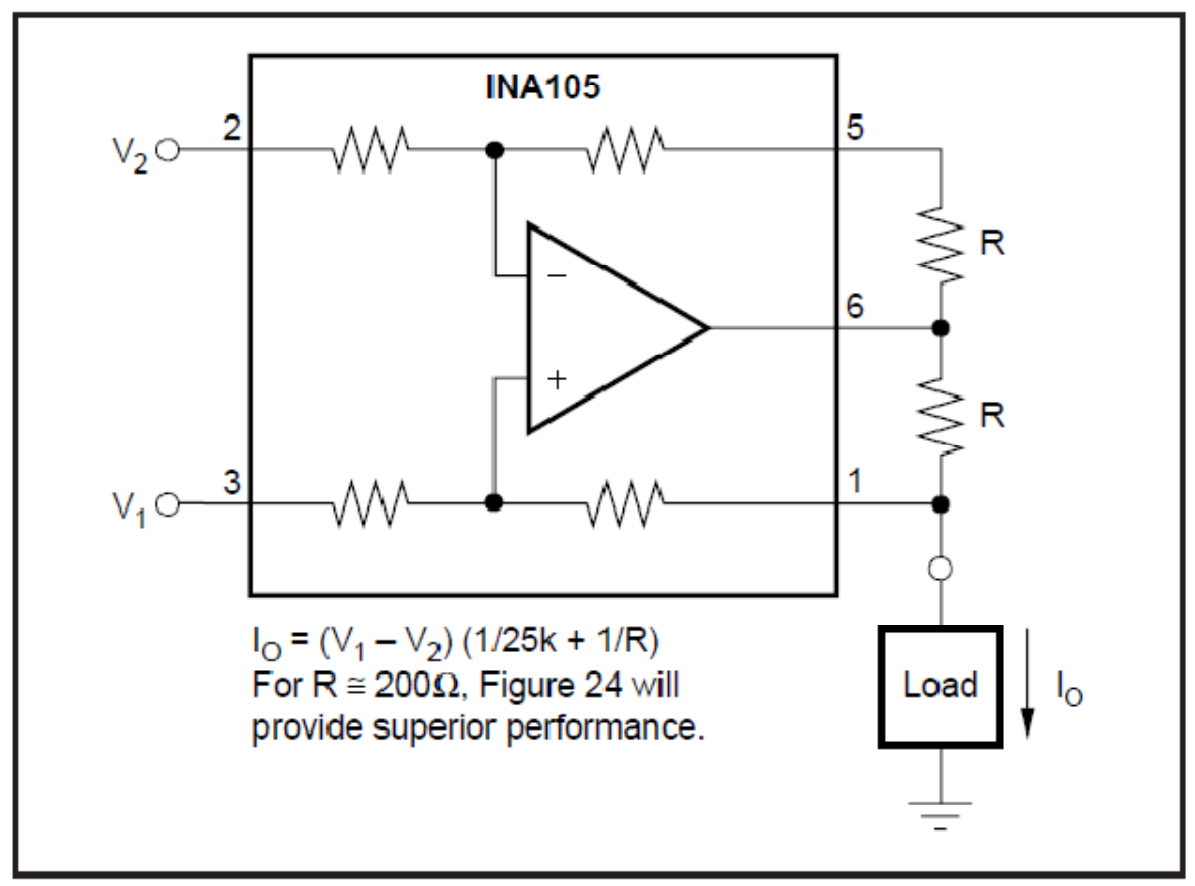

Figure 20 - Precision voltage to current converter with digital inputs

\subsubsection{GVS Device notes}

Figure 21 plots a sample GVS stimulation to induce the sensation of rolling right in a subject under bilateral bipolar stimulation. Electrode 1 is depicted on the channel 1 waveform and electrode 3 is depicted on the channel 2 waveform, both shown as voltage vs. time. The waveforms were obtained using a $2 \mathrm{~K} \Omega$ resistor as a load, recall the electrode configuration in Figure 5 when thinking about inducing the sensation of roll. 


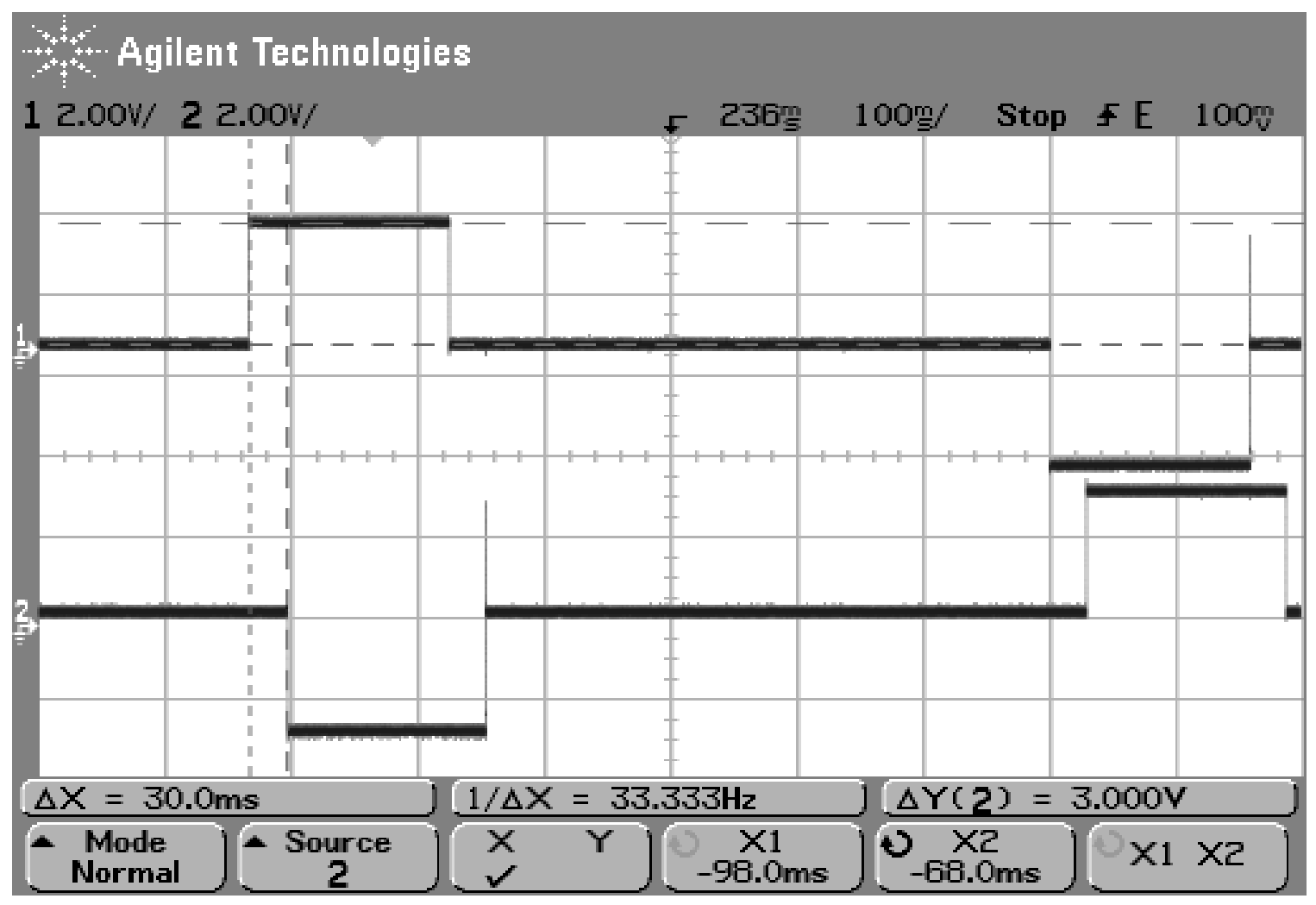

Figure 21 - Sample roll right stimulation. Channel 1 corresponds to electrode 1 and Channel 2 corresponds to Electrode 3. The stimulation was recorded at 1.5 milliamps across a $2 \mathrm{~K} \Omega$ resistor.

The roll right stimulation shown in Figure 21 was recorded at 1.5 milliamps across a $2 \mathrm{~K} \Omega$ resistor. The device delivers the desired stimulations within the current and voltage ratings discussed earlier. However, if the batteries are not fully charged the performance of the device degrades. Figure 22 shows an example of a roll left stimulation with batteries that are not fully charged. 


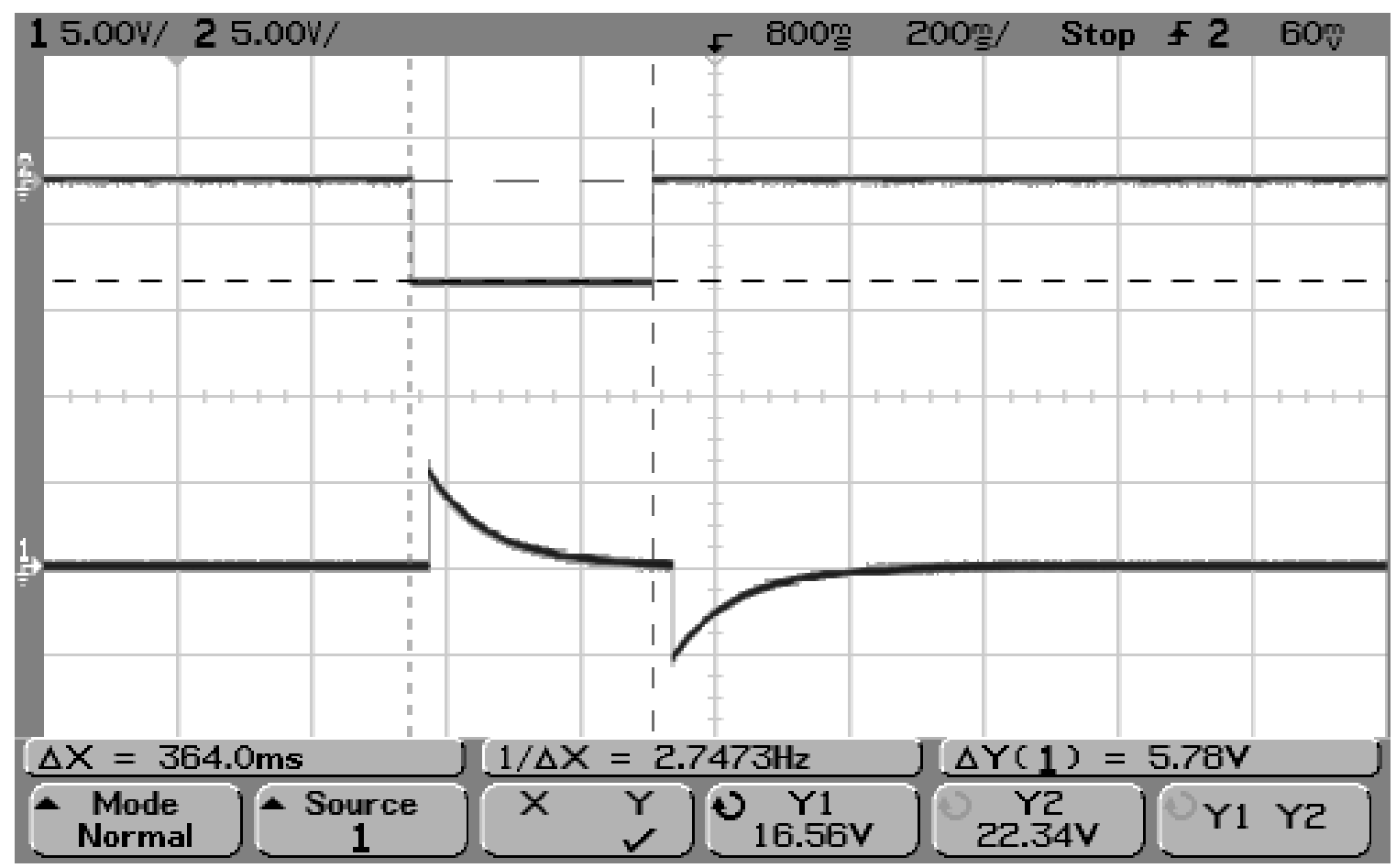

Figure 22 - Sample roll left stimulation with not fully charged batteries. Channel 2 is Electrode 1 and Channel 1is Electrode 2. 300 ms Pulse width. (Roll left, 3k resistor, 32 ms delay)

Figure 22 achieves the correct peak stimulations but Electrode 3 (Channel 1) exponentially decays, and at the end of the pulse Electrode 3 shoots negative. Most likely the exponential decay of the voltage is happening due to a capacitor charging. The spike negative after the electrode turns off results from the capacitor reversing polarity and discharging. It is likely that one or more of the batteries are not charged enough to maintain the correct voltage and are behaving like capacitors rather than battery sources. The capacitive behavior does not exist when the batteries are fully charged. It is very important that fully charged batteries are used to ensure proper stimulation.

The two pulses (one in each waveform) shown in Figure 21 and Figure 22 are required to generate roll right and left sensations, respectively. In the system implementation, the electrode stimulations on each mastoid process do not happen 
at exactly the same time. There is a 32 millisecond delay as a result of sending successive commands through the serial connection. In other words the stimulating sensations can be updated at most every 32 milliseconds. This square waveform, with varying pulse widths and amplitudes, is the stimulation waveform used for all of the tests.

\subsection{GVS Software Design Overview}

The next functional block in the system is the GVS Software. The multithreaded program simultaneously can access data from the GVS, the Graphic User Interface (GUI), and the X-Plane software. The program runs on a Windows platforms using Microsoft's .NET environment. The software consists of three main threaded components: the GVS thread, the Galvanic Vestibular Stimulator Graphic User Interface (GVS GUI) thread, and the User Datagram Protocol (UDP) thread shown in Figure 23.

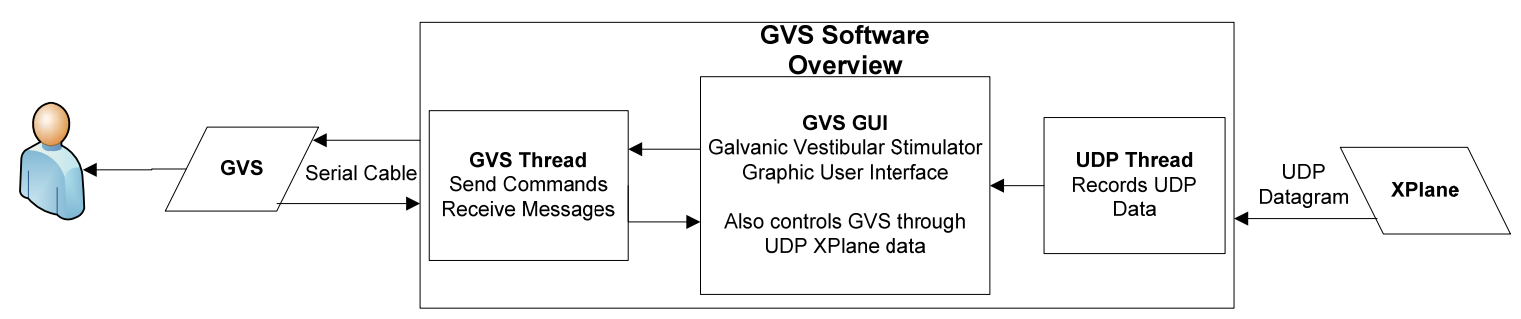

Figure 23 - Top level GVS Software overview

The GVS GUI thread is the main thread for the program. The thread controls the GVS thread and the UDP thread. Figure 24 shows a screenshot of the entire GUI. 


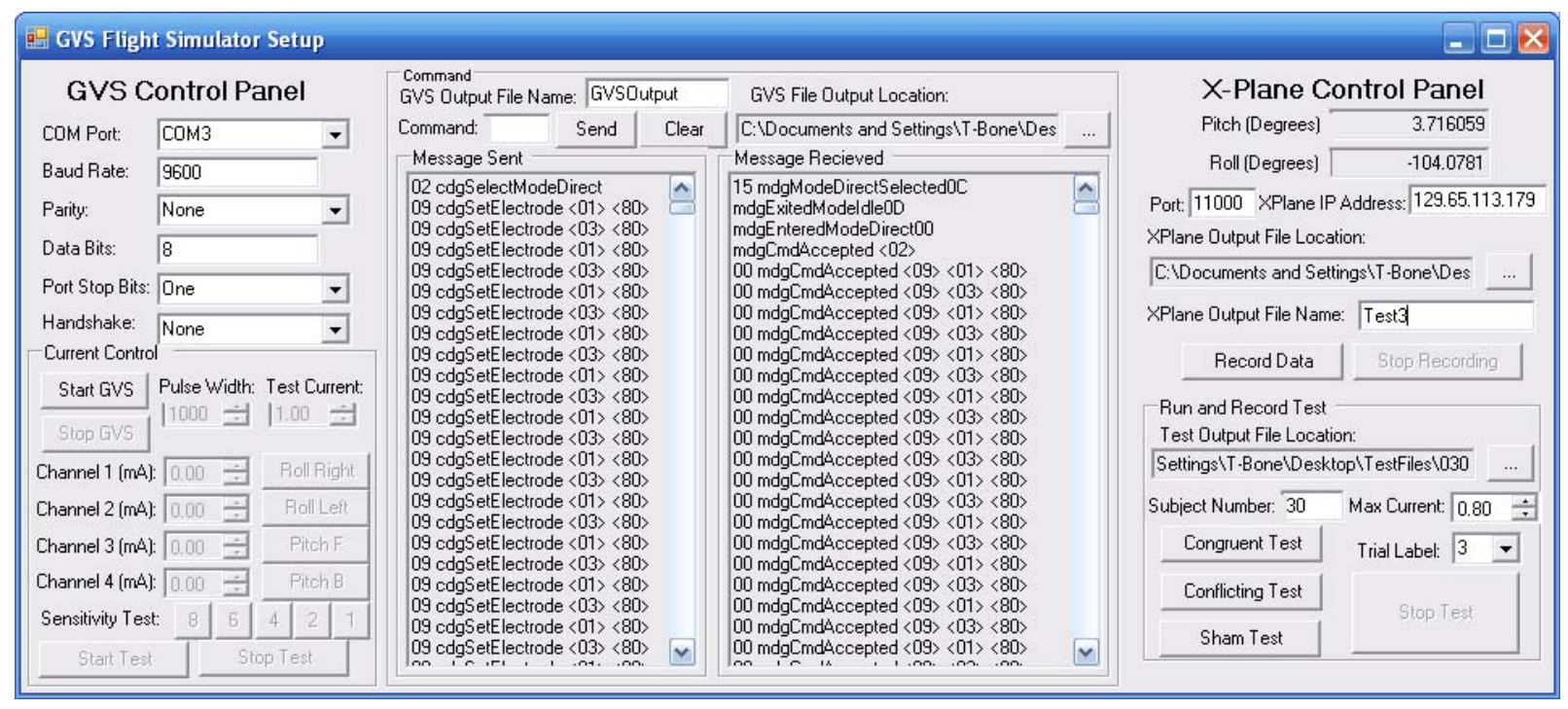

Figure 24 - Galvanic Vestibular Stimulator Graphic User Interface (GVS GUI)

In addition to controlling the GVS thread and the UDP thread program, the GVS GUI provides the functionality for performing each of the experiments in this project. Section 4.4 goes into detail about the Motion Sensitivity design and how the software supports that experiment and section 4.5 provides information about the X-Plane tests.

\subsubsection{GVS Thread}

The GVS thread sends commands to and receives messages from the GVS device, including the initial connection settings of the RS-232 port. Figure 25 shows the default RS-232 connection settings for the GVS device. These settings automatically populate the user interface. The baud rate is 9600 bits per second. There is no parity for error checking. There are 8 data bits for each unit of transmission. There is one stop bit. The experimenter needs to assign the correct communication (COM) port in the drop down menu. 


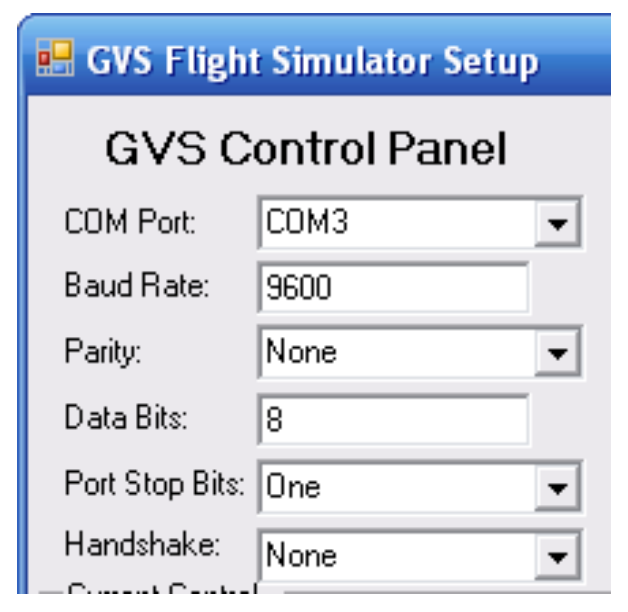

Figure 25 - GVS device default RS-232 connection settings.

The middle section of the GVS GUI is shown in Figure 26. The middle text fields update with commands sent to and messages received from the GVS as the stimulations are happening. These messages and commands are also logged to a text file. The text file is saved to the location specified in the "GVS File Output Name" field shown in Figure 26. The text file location is specified by the "GVS File Output Location" field. The default filename is "GVSOutput.txt" and it is saved to the desktop. 


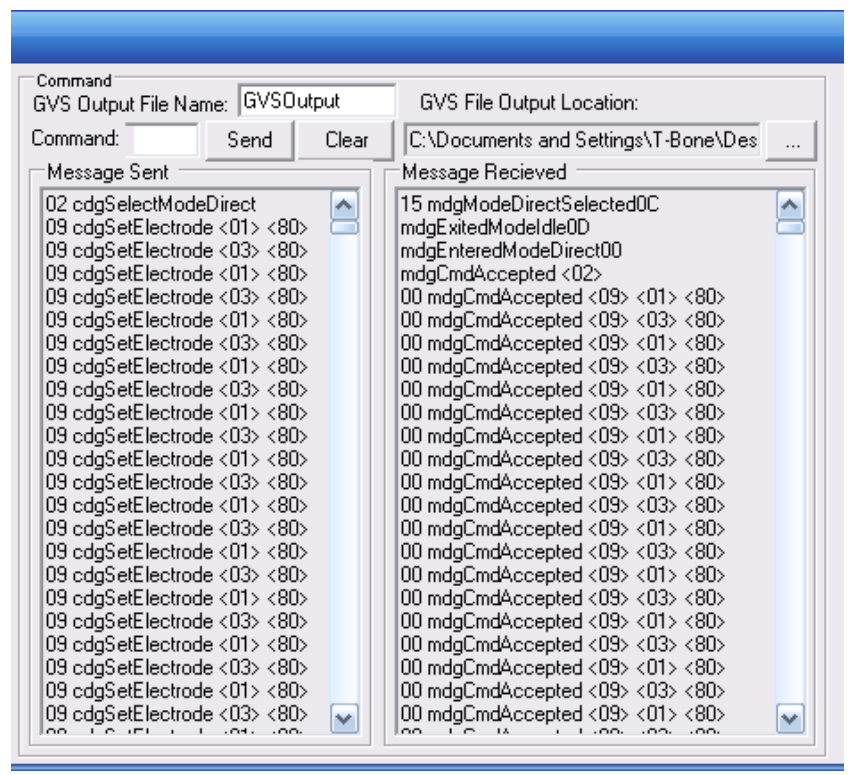

Figure 26 - Middle section of GUI: Output filename and location and the message and command textboxes.

\subsubsection{UDP Thread}

The UDP thread manages all of the communication between the program and X-Plane. The top right portion of the GUI shown Figure 27 provides settings for the UDP Thread.

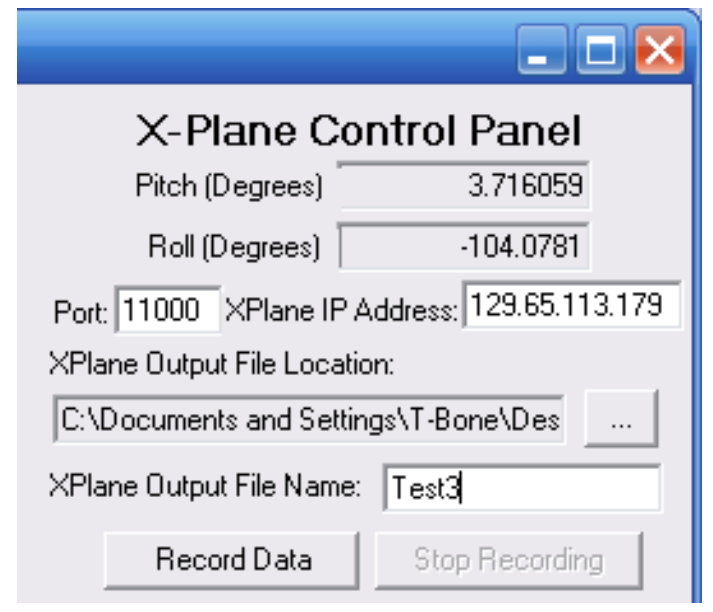

Figure 27 - X-Plane data record setup 
The program will automatically output the data received from XPlane to a text file. The text file name is specified in the "XPlane Output File Name" field shown in Figure 27. The text file is stored at the location specified by the "XPlane Output File Location" field. The filename and file location should be selected prior to beginning to record data.

In order to begin receiving data from X-Plane the IP address of the computer running X-Plane and a port number must be entered into the GUI for the connection to be made. Likewise, X-Plane requires the IP address of the receiving computer, a port number, and specified data to output. Section 4.5 explains these configurations in further detail. With the correct port and IP address settings, selecting the "Record Data" button starts the UDP thread and makes the GVS Software start recording data. X-Plane uses UDP to send information across the Ethernet connection. UDP is faster than TCP/IP but it does not provide any error checking of the data transmitted. It sends the data with little concern about whether it reaches the destination correctly or not. The data is saved at the default desktop file location with the default "X-PLANEOutput" filename unless otherwise specified prior to beginning the recording.

The GUI also displays the pitch and roll values, which controls the stimulation commands sent to the GVS, if dataset 18 is selected. Dataset 18 is one of the data options within XPlane to output to the UDP port. Section 4.6 includes details for selecting data and Figure 39 shows all of the data options within XPlane. This dataset must be selected for stimulations to take place. 4.4 Motion Sensitivity Test System Design 
The left side of the GVS GUI consists of controls for the GVS device. After selecting the COM port and pressing "Start GVS" the control buttons shown in Figure 28 become enabled.

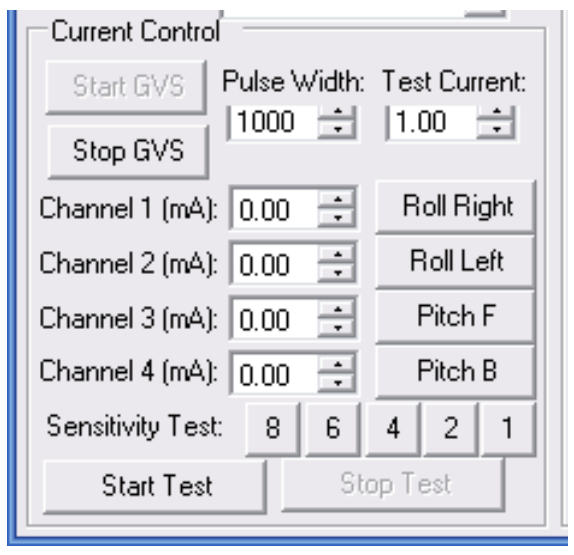

Figure 28 - GVS Current control buttons

The Current Control panel allows you to independently control the current levels of each channel or electrode. The channel number corresponds to the electrode number from Figure 5. There are also buttons that automatically generate roll right, roll left, pitch forward, and pitch backward sensations. Each of these sensations is generated through square wave pulse stimulations. The current level and pulse width of the stimulation can be adjusted. Again, the max current level allowed by the GVS and the control panel is 2.54 milliamps. The minimum value allowed is -2.56 milliamps. The pulse width can be adjusted between 1 millisecond to 5000 milliseconds (5 seconds).

Selecting the "Start Test" button at the bottom of the GVS Current control box shown in Figure 28 begins the entire motion sensitivity stimulation sequence. The GVS generates sensations of rolling back and forth. The incremental rolling 
sensations start out slowly with 8 seconds between alternating roll sensations. The stimulations roll left, roll right, roll left, and roll right with a constant 8 seconds between each roll. Then the pattern is repeated and the time period between each stimulation decreases from 8 seconds to $6,4,2$, and 1 second sequentially. Between each incremental stimulation sequence the subject indicates a status of motion sickness with the following sickness scale:

0: no symptoms;

1: any symptoms, however slight;

2: mild symptoms, e.g., stomach awareness but not nausea;

3: mild nausea;

4: mild to moderate nausea;

5: moderate nausea but can continue;

6: moderate nausea and want to stop [13].

The numbered buttons in Figure 28 above the "Start Test" button allow functionality for administering $8,6,4,2$, and 1 second rolling stimulation sequences individually in addition to automatically administering the entire test. Figure 29 shows the time delay between each successive stimulation of the Motion Sensitivity Test and Figure 30 shows the corresponding stimulation sequence recording the electrode activity on the oscilloscope. 


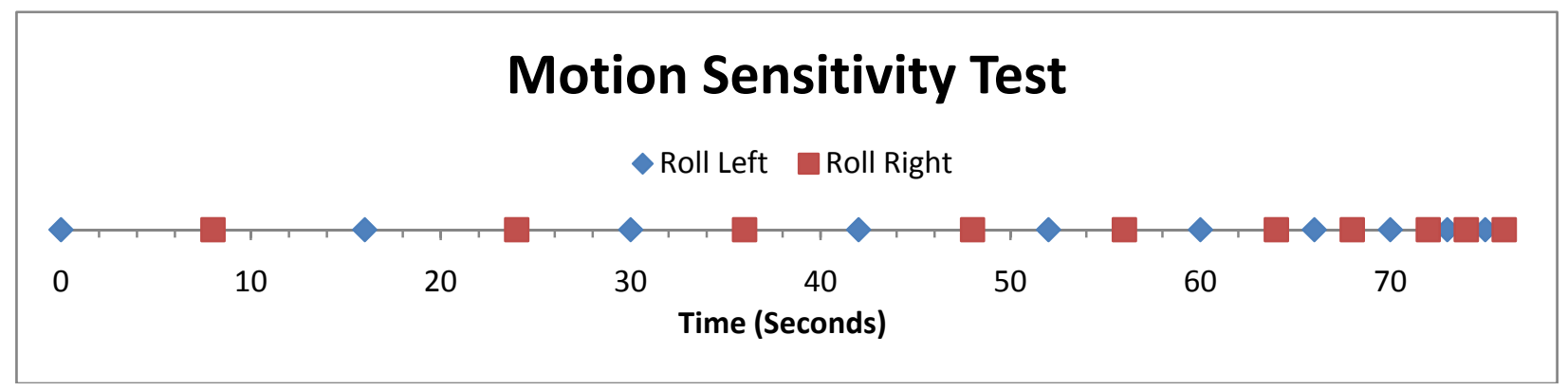

Figure 29 - Motion Sensitivity Test. Blue dot represents a roll left sensation and a red dot represents s roll right sensation.

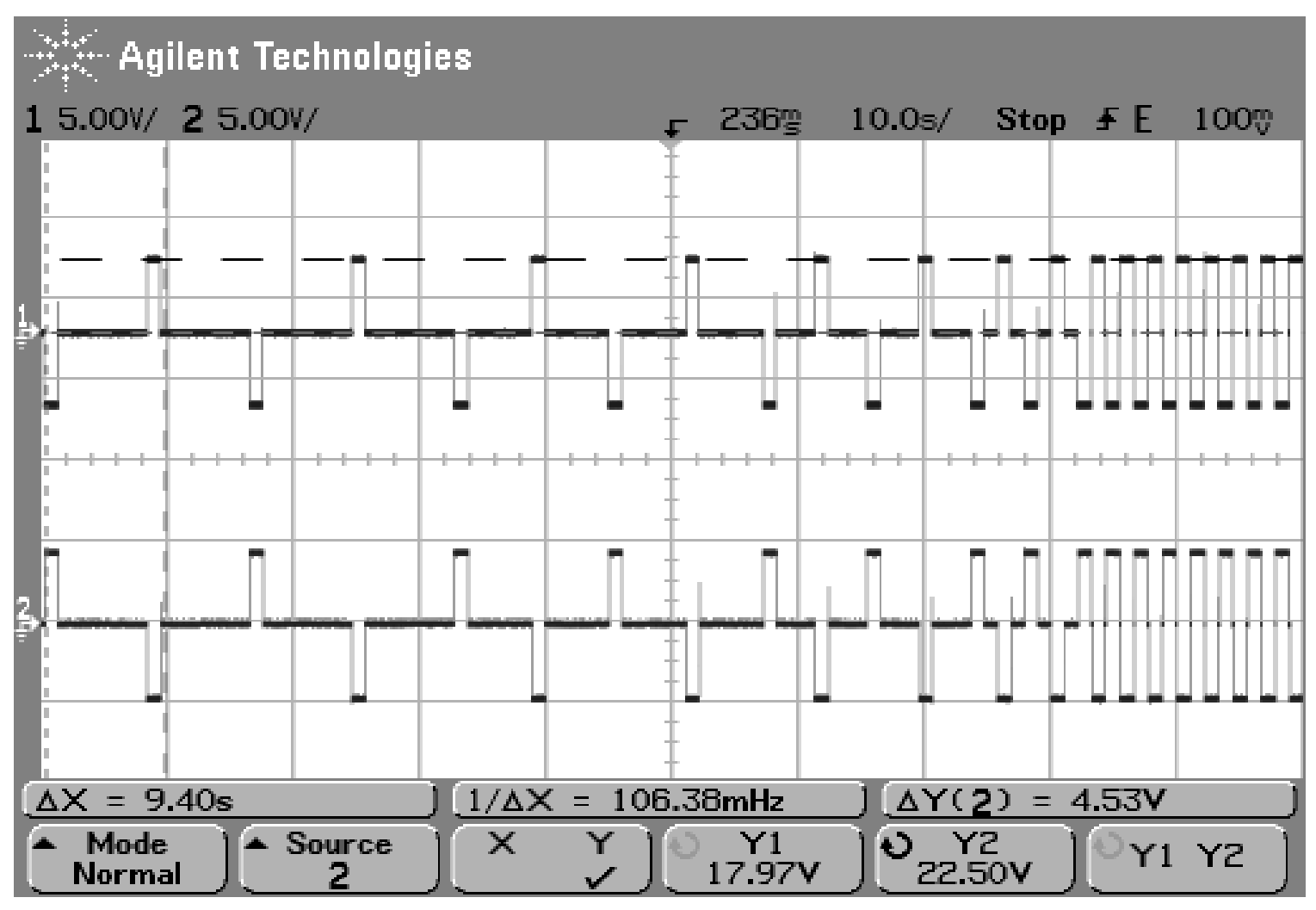

Figure 30 - Lab Recording of Motion Sensitivity Test: 1 milliamp 1 second pulses

Each square dot in Figure 29 represents a stimulation. The roll left begins the stimulation sequence represented by a blue dot. Eight seconds later a roll right sensation occurs represented by the red dot. For a given time duration, say eight seconds, there will be a one second pulse followed by seven seconds with no stimulation. The roll left sensation followed by a roll right stimulation generates a 
rocking sensation, which is sped up as the test progresses. When the stimulations are 1 second long, the final stimulation has no time between the roll right and rock left stimulations. The one second sequence continues until the administrator stops the test.

As will be explained in the procedures of chapter 5 , each subject completes a Motion Sickness Question (MSQ) prior to any testing. The MSQ, developed by Robert Kennedy [16], provides a standardized motion sickness score for each subject. The scoring in this experiment used Motion Sickness Susceptibility Questionnaire (MSSQ). The scoring is broken up into two parts MSSQA, representing the sickness score prior to age 12, and MSSQB, representing the sickness score in the last 10 years. These scores were calculated using the following equations:

$$
\begin{aligned}
\text { MSSQA } & =\frac{2.64 \times(\text { total sickness score child }) \times 9}{(\text { number of types experienced as a child })} \\
\text { MSSQB } & =\frac{2.64 \times(\text { total sickness score adult }) \times 9}{(\text { number of types experienced as an adult })}
\end{aligned}
$$

The raw motion sensitivity score is equal to MSSQA + MSSQB [12]. The higher the score the more susceptible an individual is to motion sickness. These scores provide data to compare with the sickness scores resulting from the rocking sensations generated during the motion sensitivity test. 


\subsection{X-Plane Flight Simulator System Design}

The final area of the GUI is the Run and Record Test on the bottom right hand corner of the GUI shown in Figure 31. The run and record portion of the GUI provides the functionality for the GVS stimulations coupled with a visual stimulus.

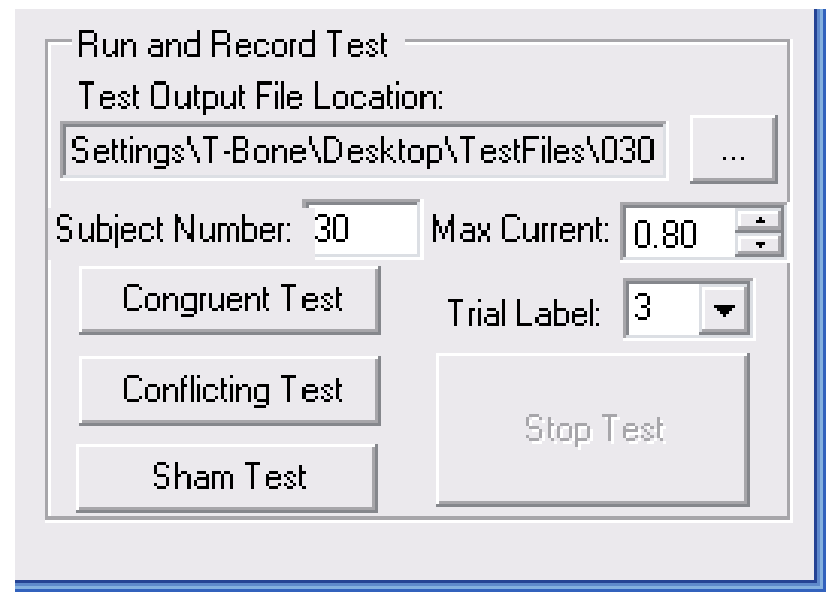

Figure 31 - Run and Record Test

The GUI requires a subject number, trial number, and a Max current value to be selected. The trial number corresponds to which task profile the subject is performing: Test 1 (VS-A), Test 2 (VS-C), or Test 3 (Chase plane). Each test profile is explained in Table 1 of section 4.5.2. Both the subject number and the trial number entries are used for naming the automatically generated output files. In running one of the tests, the GVS-sent commands, received messages, and the X-Plane recorded data are automatically saved to the Desktop or a previously selected file location specified by the "Test Output File Location" field in Figure 31. The experimenter must also enter in the maximum current level to be used during stimulations. After entering in a subject number, trial number, and 
max current, the buttons for the congruent, conflicting, and sham test are enabled. The congruent, conflicting, and sham stimulation profiles are explained in section 4.5.3. During the test, the test button selection turns green and the stop button turns red. The stop button is the only button enabled while these tests are running. In addition, when the test is running, the pitch and roll values automatically show up in the designated fields on the GUI. The commands and messages from the GVS also populate the associated text boxes on the GUI.

\subsubsection{Flight Simulator Setup}

Figure 32 shows the dimensions for the flight simulator setup. In this test, it was important to try to minimize visual references outside of the flight simulator. The simulator image was projected on a wall in a dark room with no windows.

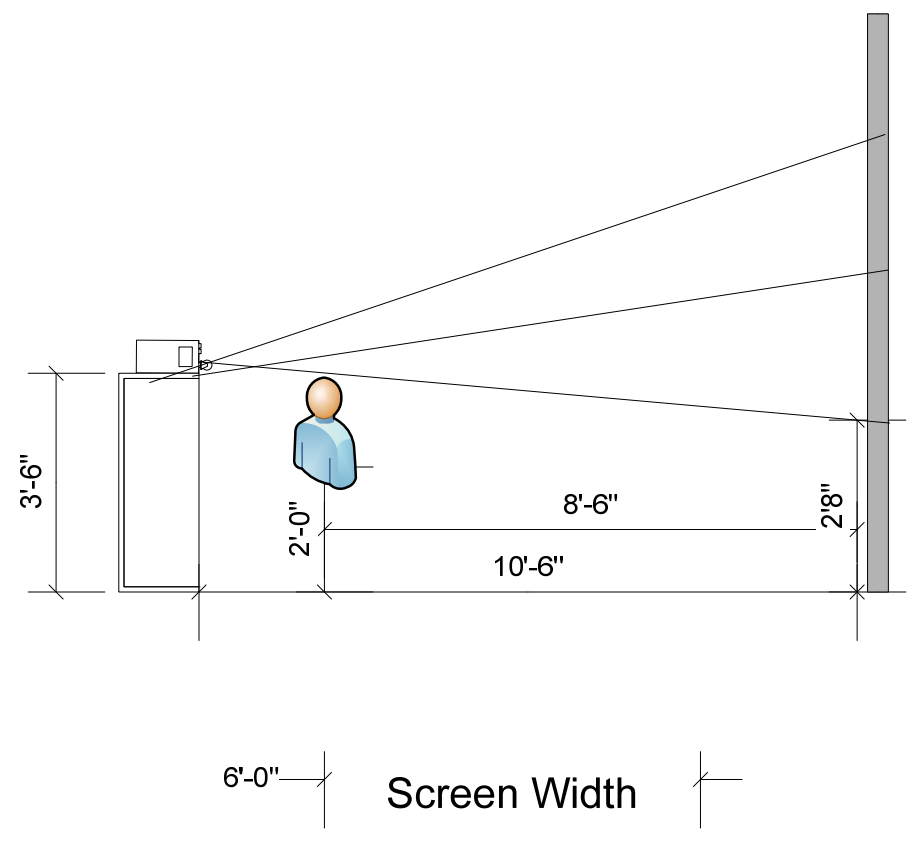

Figure 32 - Flight simulator room dimensions 
The subject is placed 8 feet 6 inches from the wall and the subject sits 2 feet off the ground. The projector is 2 feet behind the subject and 3 feet 6 inches off the ground. The projected image is 2 feet 8 inches off the ground and 6 feet wide. This setup provides a large visual stimulus for the subject while they complete various tasks. Figure 33 shows the actual room of the flight simulator.

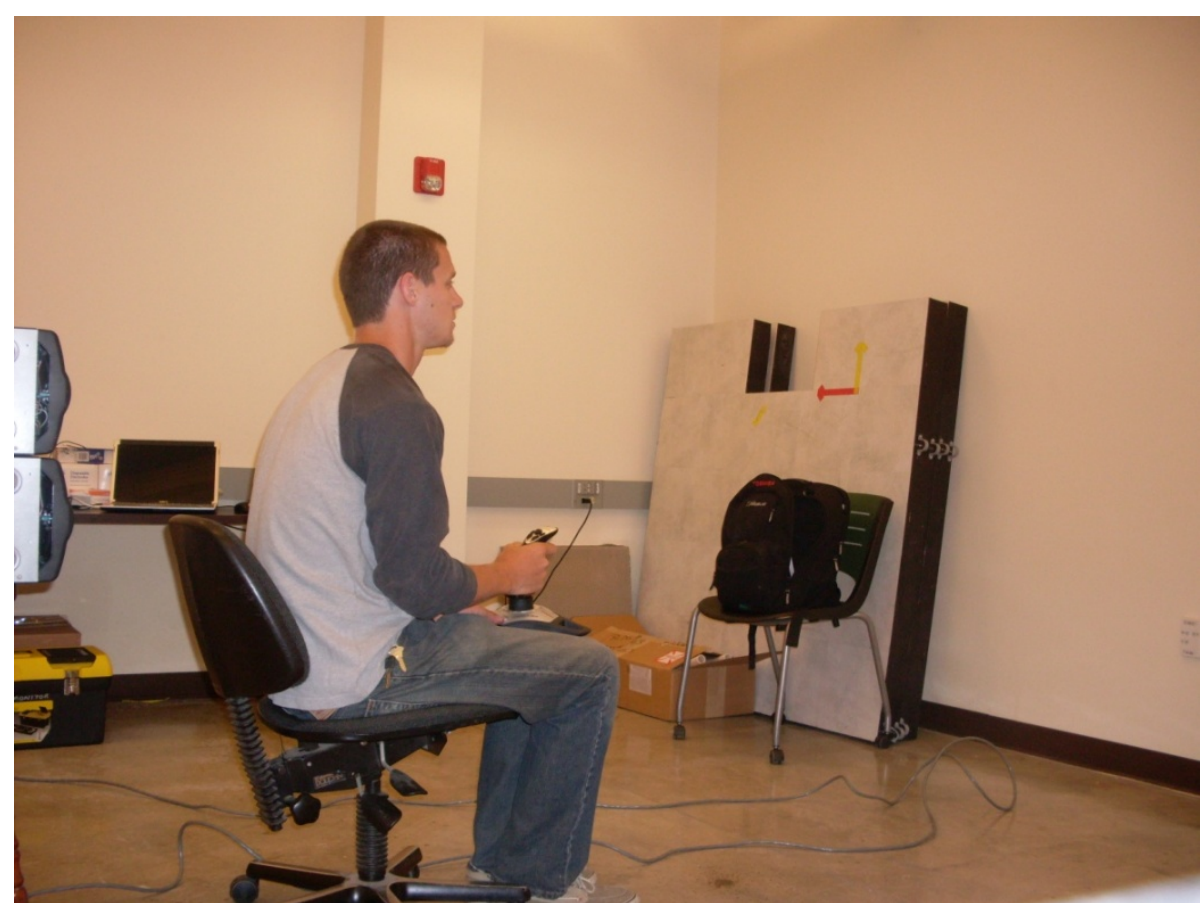

Figure 33 - Sample flight simulator setup

The subject sits on the chair with the video projected on the wall. The test is performed with the lights out.

\subsubsection{X-Plane Maneuver Series}

Each subject completes the three X-Plane flight simulator tasks explained in Table 1: VS-A, VS-C, and follow a Chase Plane. The VS-A and VS-C tasks are modeled off of actual flight training maneuvers in the Federal Aviation Administration Instrument Flying Handbook [25]. The tasks are designed to 
orient each subject with the common flight instruments and provide basic flight training. Table 1 describes each task in detail.

\begin{tabular}{|l|l|l|}
\hline VS-A: & VS-C: & Chase Plane: \\
\hline Heading: North & Heading: Changes with bank & The final test is to follow the \\
Airspeed: $100-200$ Knots & Airspeed: Changes & path a chase plane. \\
Altitude (changes $+/-1,000$ & Altitude (changes $+/-1,000$ & \\
$\mathrm{ft}$ ) & ft) & \\
Bank (constant at 0$)$ & Bank $\left(+/-30^{\circ}\right.$ ) & \\
Task: & Task: & \\
-Start at $4,000 \mathrm{ft}$ and climb to & - Start at $4,000 \mathrm{ft}$ and climb to & \\
$5,000 \mathrm{ft}$ at pitch angle of & $5,000 \mathrm{ft}$ at pitch angle of & \\
$10^{\circ}$ to $30^{\circ}$ degrees. & $10^{\circ}$ to $30^{\circ}$ degrees, & \\
-Hold $5,000 \mathrm{ft}$ for 15 seconds & maintain $30^{\circ}$ bank right. & \\
-Decrease altitude back down & -Level aircraft. \\
to $4,000 \mathrm{ft}$ at pitch angle & -Decrease altitude back down & \\
of $-10^{\circ}$ to $-30^{\circ}$. & to $4,000 \mathrm{ft}$ at pitch angle & \\
-Hold $4,000 \mathrm{ft}$ for 15 & of $-10^{\circ}$ to $-30^{\circ}$ & \\
seconds. & maintain a $30^{\circ}$ bank right. & \\
-Repeat sequence. & -Hold $4,000 \mathrm{ft}$ for 15 & \\
& seconds. & \\
& -Level aircraft and repeat & \\
& sequence with $30^{\circ}$ bank & \\
& left. & \\
\hline
\end{tabular}

Table 1 - VS-A, VS-C, and chase plane task profiles

\subsubsection{Congruent, Conflicting, and Sham Stimulation Profiles}

During the tasks, the GVS stimulates congruent, conflicting, and sham sensations to each of the electrodes shown in Figure 5. The congruent stimulation profile stimulates sensations congruent to the roll and pitch angles of the aircraft. If the aircraft rolls right, the subject is stimulated to feel as if they are actually rolling to the right. The conflicting profile stimulates sensations conflicting with the roll and pitch of the aircraft. If the aircraft rolls right the subject is stimulated to feel as if they are rolling to the left. The Sham test provides a stimulation so that the subject can feel current entering the body but at low enough amperage to generate any sensations. The three profiles are administered in random orders for 
each flight task to mitigate training effects, since task proficiency typically increases with repetition. The threshold for both the roll and pitch sensations is +/- 10 degrees. If the roll or pitch angle exceeds the threshold a corresponding stimulation begins until the angle drops below the threshold. Figure 34 plots the roll values and Figure 35 plots the pitch values generated from the X-Plane Chase Plane test discussed in Table 1 and the corresponding congruent, conflicting, and sham stimulations. 


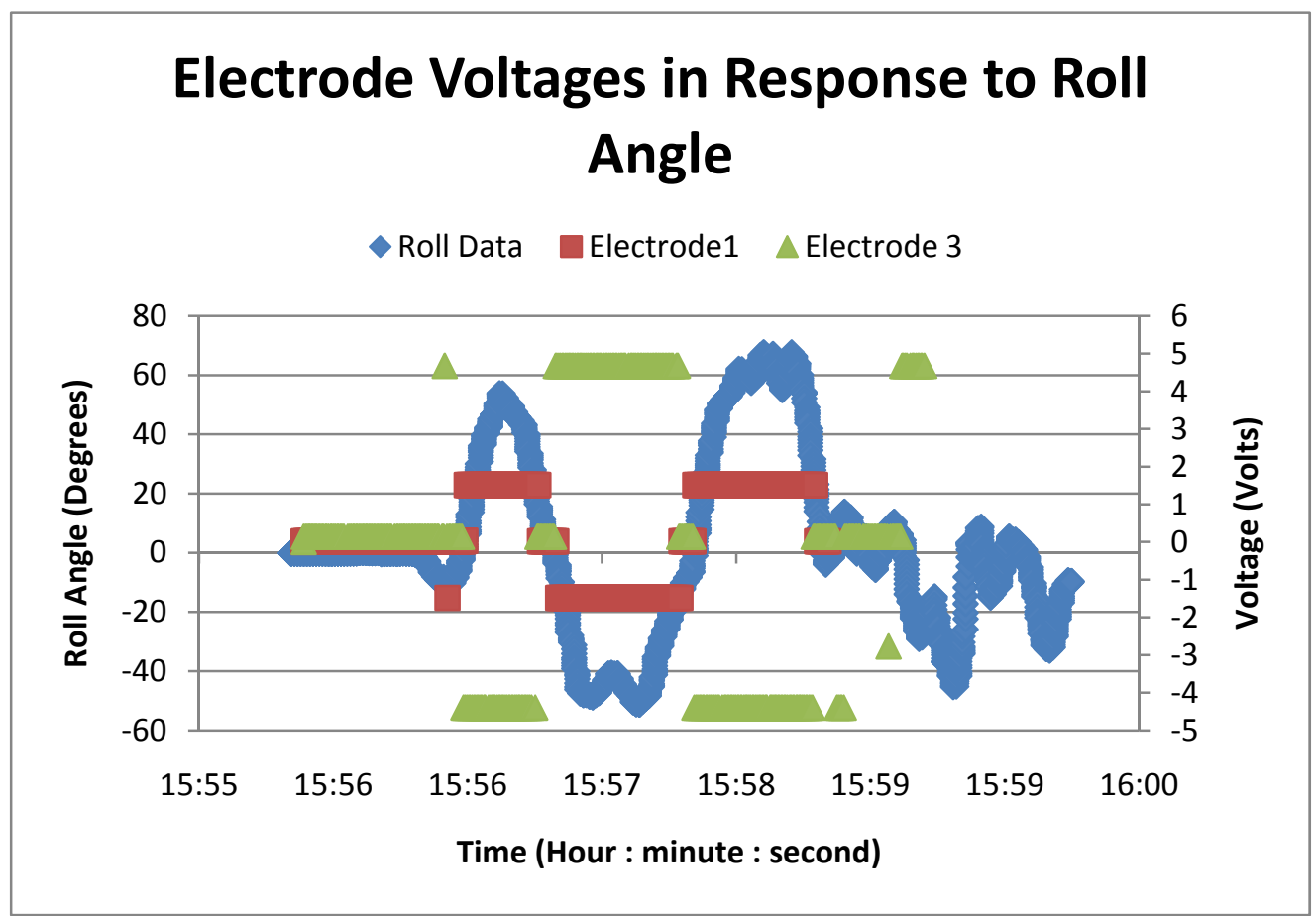

(a) Congruent Test Roll stimulations

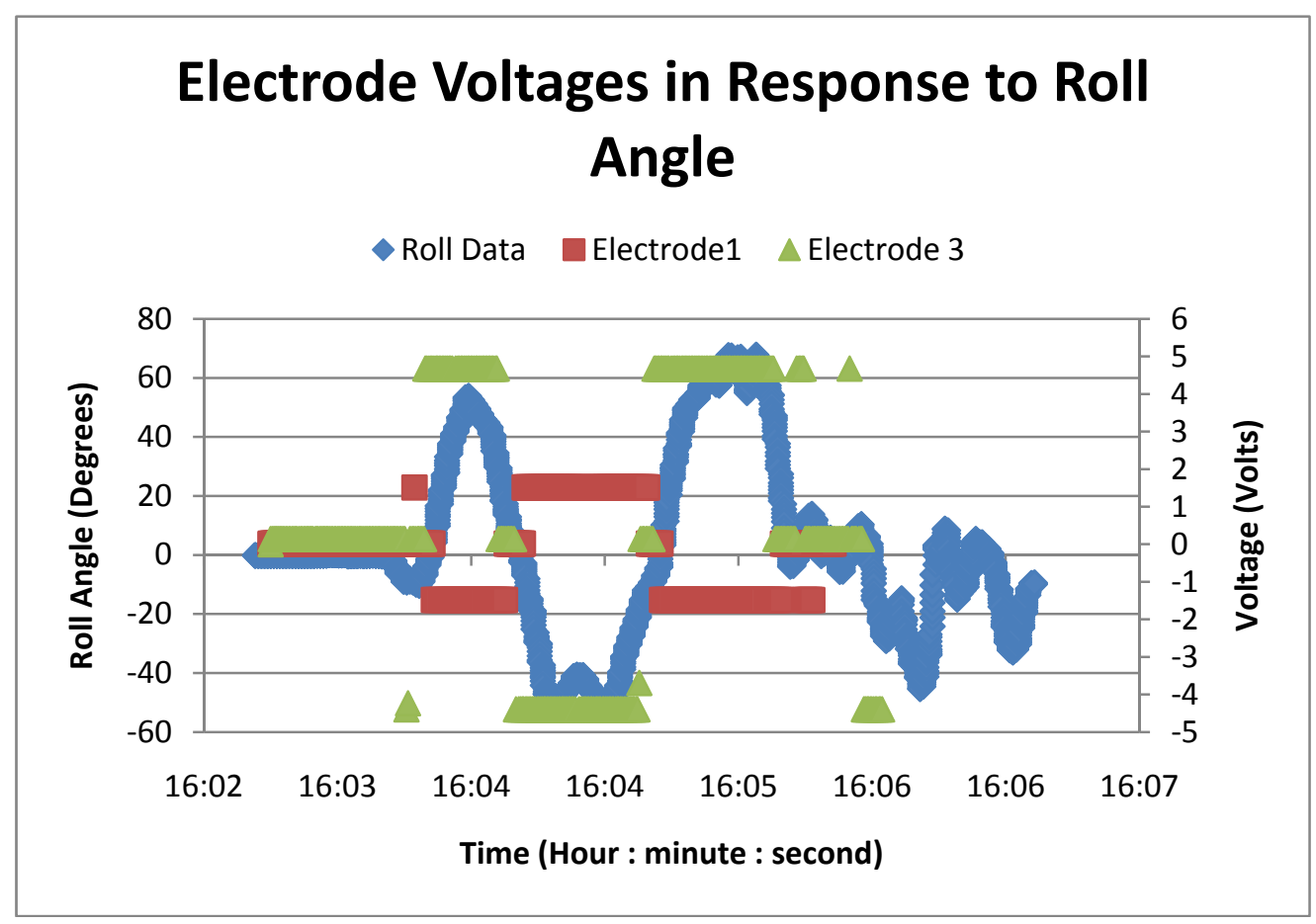

(b) Conflicting Test Roll Stimulations 


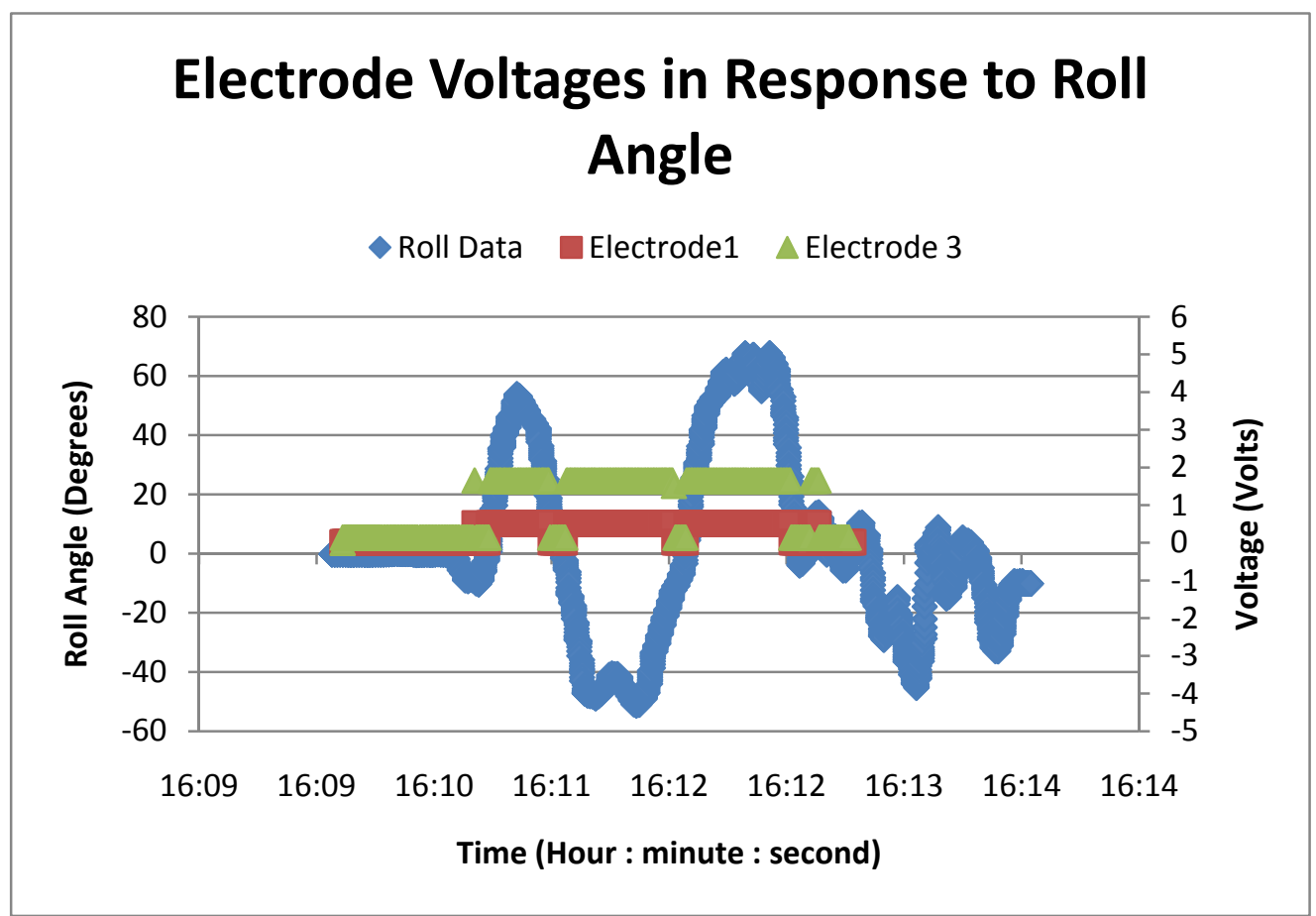

(c) Sham Test Roll Stimulations

Figure 34 - Roll stimulations generated from Chase Plane test. The Congruent and Conflicting stimulations are at 1.5 milliamps and the Sham test is at 0.5 milliamps. Electrode 1 voltage was measured across a $1 \mathrm{~K} \Omega$ resistor and Electrode 3 voltage was measured across a $3 \mathrm{~K} \Omega$ resistor. (a) Congruent Roll Stimulations (b) Conflicting Roll Stimulations (c) Sham Roll Stimulations

In Figure 34 the roll angles of the chase plane are on the left vertical axis and the associated electrode voltages are on the right vertical axis. A change in roll angle only activates Electrode 1 and Electrode 3.The Congruent and the Conflicting stimulations are at 1.5 milliamps and the Sham stimulation is at 0.5 milliamps. The sham test is always one-third the max current selection. To test the performance of the system in the lab for generating roll sensations, Electrode 1 and Electrode 3 voltages for the congruent, conflicting, and sham stimulation profiles were measured across known resistances. The Electrode 1 voltage is measured across a $1 \mathrm{~K} \Omega$ resistor and the Electrode 3 voltage is measured across a $3 \mathrm{~K} \Omega$ resistor. In the Congruent Test shown in Figure 34(a), if the plane rolls 
right, a positive roll angle, the right electrode provides a -4.5 voltage and the left electrode generates a 1.5 voltage. The voltage polarities are reversed for roll left, a negative roll angle, sensation. In the Conflicting Test shown in Figure 34(b), if the plane rolls right, a positive roll angle, the right electrode stimulates a 4.5 voltage and the left electrode generates a -1.5 voltage. These voltages are opposite the congruent test and therefore generate a conflicting sensation. In the Sham Test shown in Figure 34(c), if the plane rolls right or left Electrode 1 provides a 0.5 volts and Electrode 3 generates a 1.5 volts. Again, Figure 35 shows the electrode current levels in response to the pitch angles.

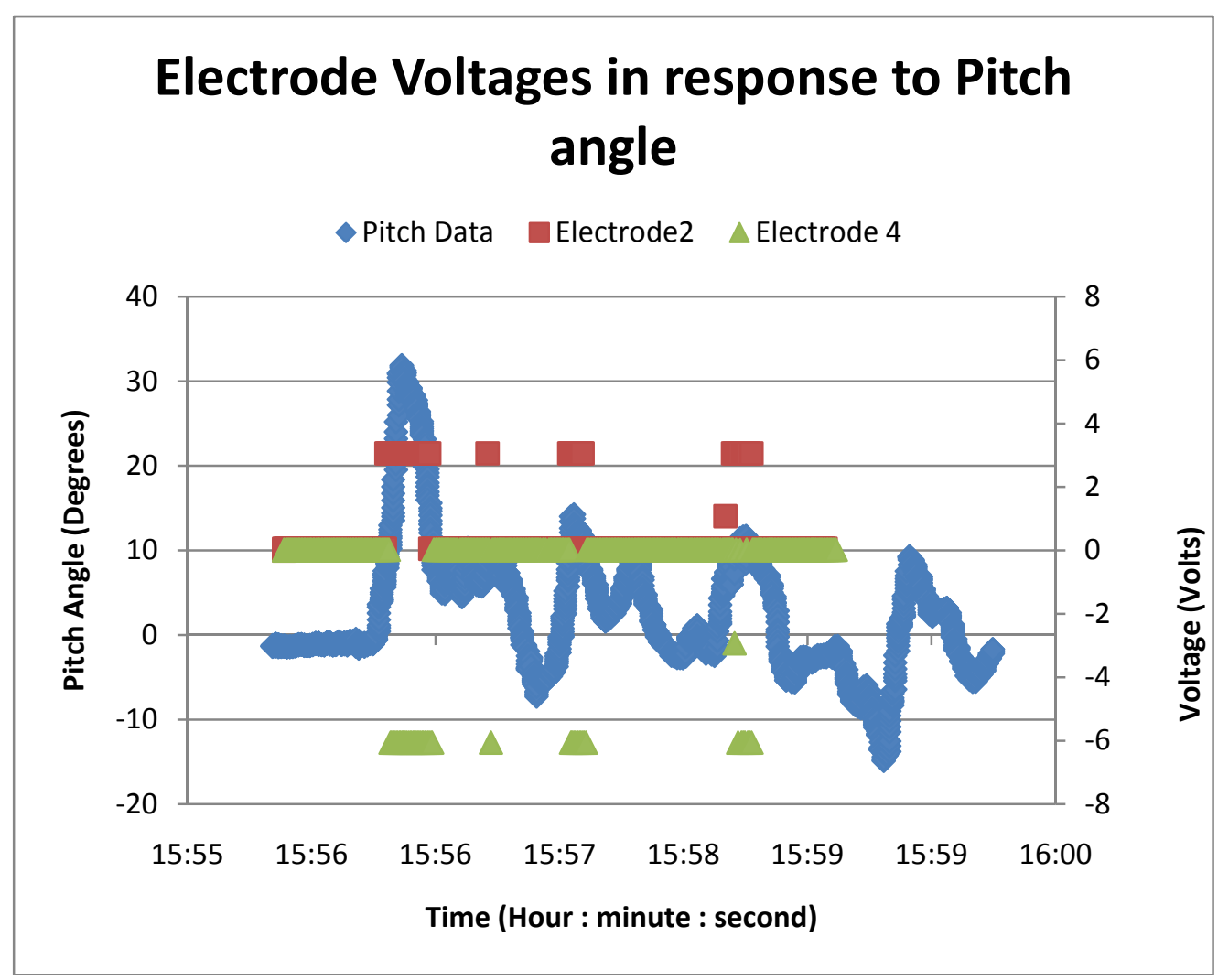

(a) Congruent Test Pitch Stimulations 


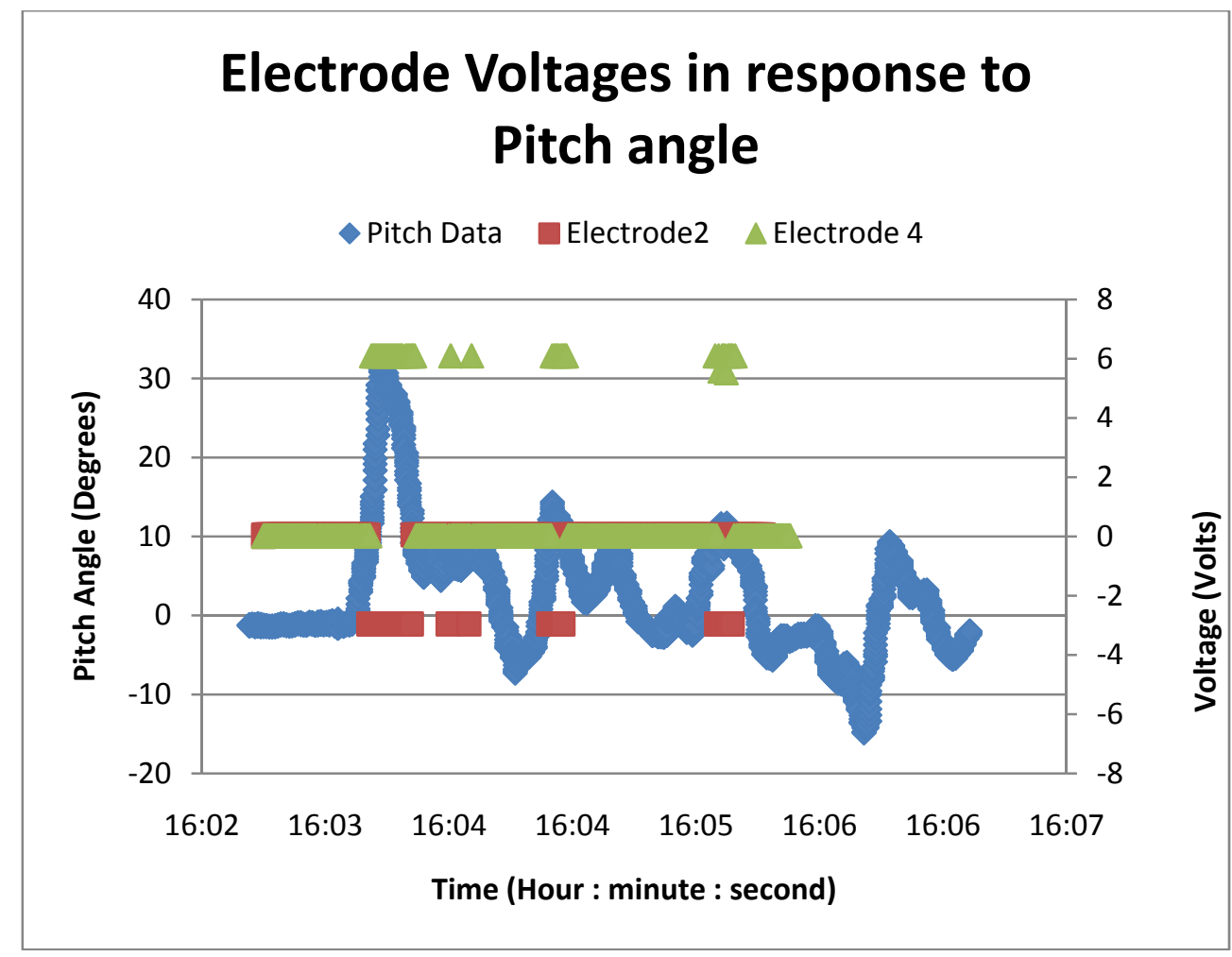

(b) Conflicting Test Pitch Sensations

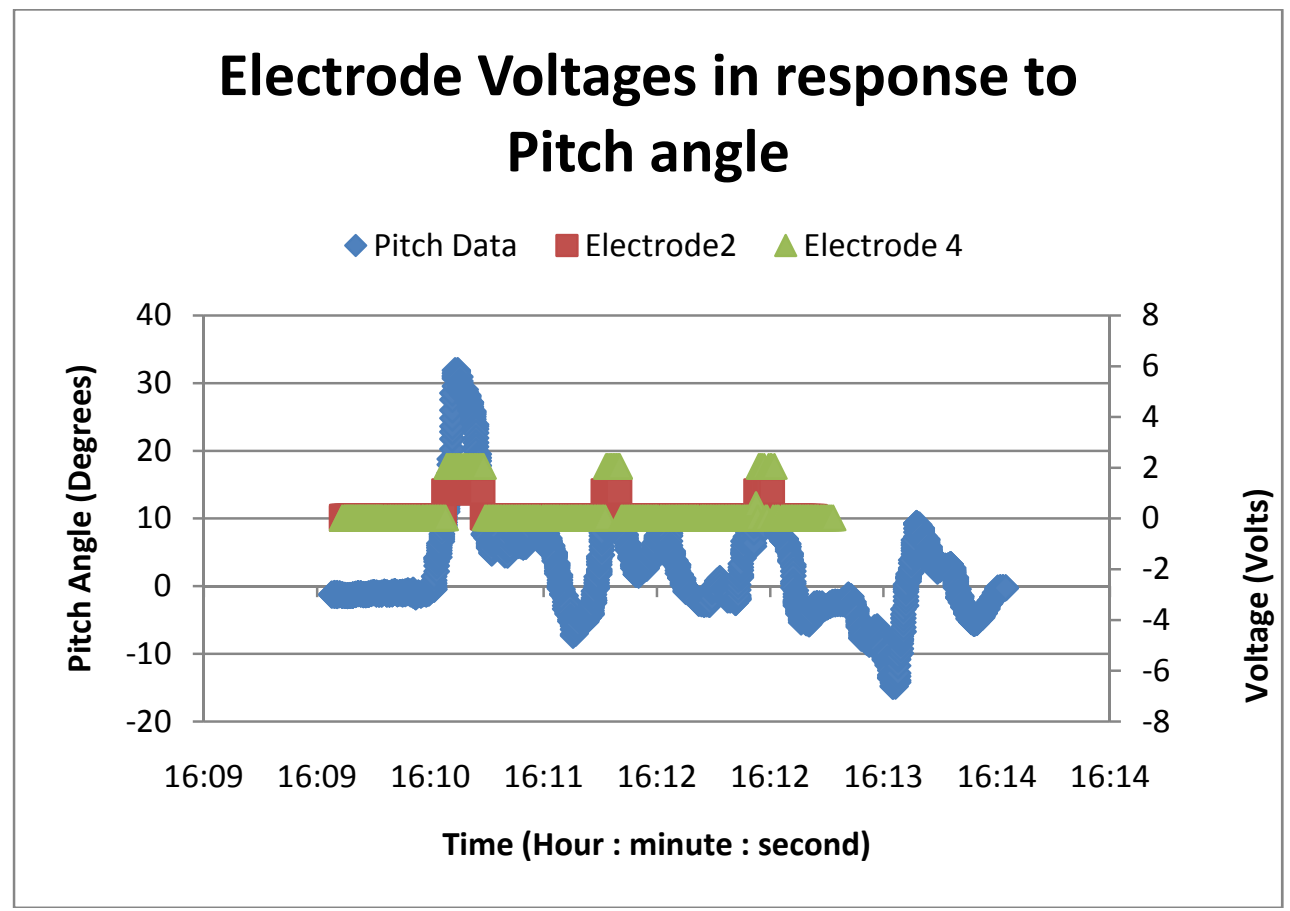

(c) Sham Test Pitch Sensations

Figure 35 - Pitch stimulations generated from Chase Plane test. The Congruent and Conflicting stimulations are at 1.5 milliamps and the Sham test is at 0.5 milliamps. Electrode 1 voltage was measured across a $1 \mathrm{~K} \Omega$ resistor and Electrode 3 voltage was measured across a $3 \mathrm{~K} \Omega$ resistor. (a) Congruent Roll Stimulations (b) Conflicting Roll Stimulations (c) Sham Roll Stimulations 
In Figure 35 the pitch angles of the chase plane are on the left vertical axis and the associated electrode voltages are on the right vertical axis. A change in pitch angle only activates Electrode 2 and Electrode 4. The Congruent and the Conflicting stimulations were at 1.5 milliamps and the Sham stimulation was at 0.5 milliamps. To test the performance of the system in the lab for generating pitch sensations, Electrode 2 and Electrode 4 voltages for the congruent, conflicting, and sham stimulation profiles were measured across known resistances. The Electrode 2 voltage was measured across a $2 \mathrm{~K} \Omega$ resistor and Electrode 4 voltage was measured across a $4 \mathrm{~K} \Omega$ resistor. In the Congruent Test shown in Figure 35(a), if the plane pitches downward, a negative pitch angle, the forehead electrode stimulates -3 volts and the neck electrode generates 6 volts. The voltage polarities are reversed for pitch backward, a positive pitch angle, sensation. In the Conflicting Test shown in Figure 35(b), if the plane pitches downward, a negative pitch angle, the forehead electrode stimulates 3 volts and the neck electrode generates -6 volts. These voltages are opposite the congruent test and therefore generate a conflicting sensation. In the Sham Test shown in Figure 35(c), if the plane pitches forward or backward Electrode 2 generates 1 volt and Electrode 4 generates 3 volts.

\subsubsection{X-Plane Cirrus Jet and Instrument Panel}

To complete each tasks the subject flies a Cirrus Jet, shown in Figure 33. This jet was selected because it provides a balance between control and sensitivity. 


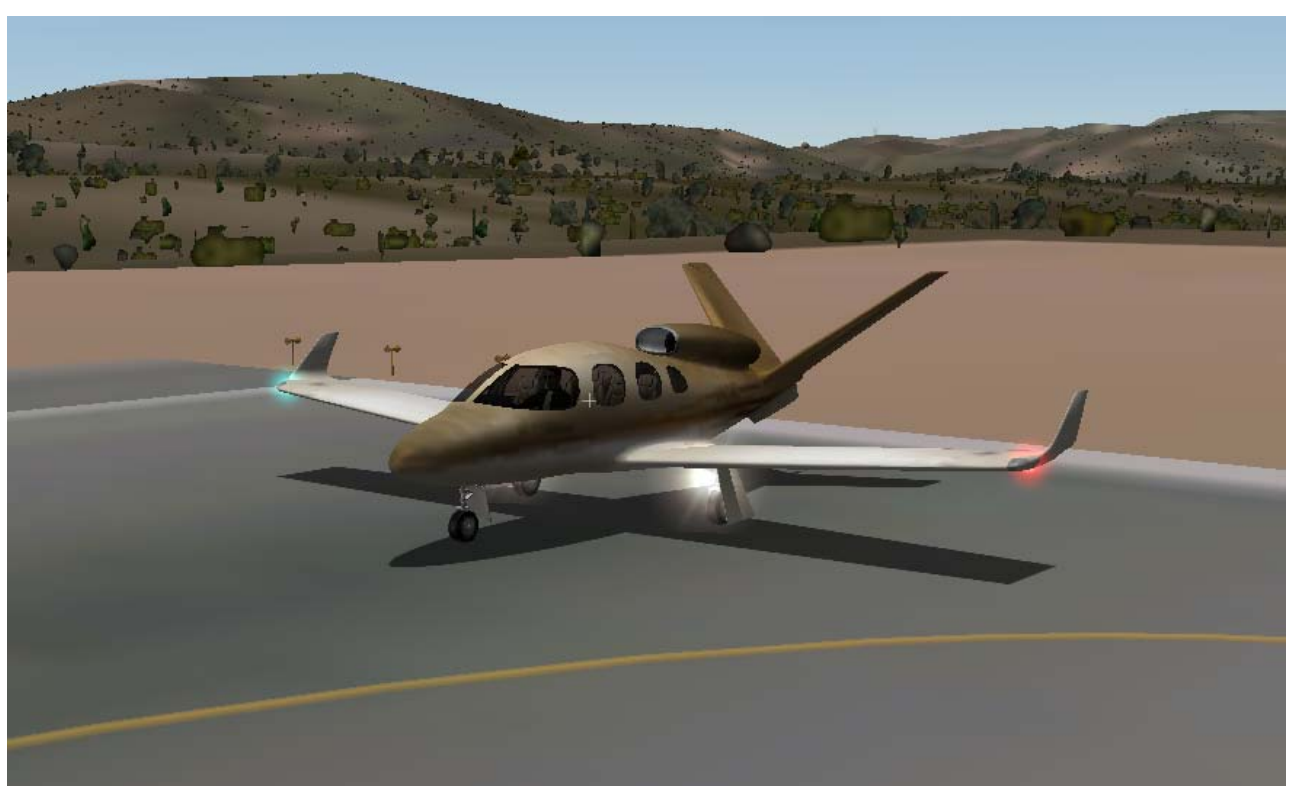

Figure 36 - Cirrus Jet used for all of the X-Plane simulator tests

To complete the first two tasks (VS-A and VS-C), the subject must rely heavily on the instrument panel of the jet. The Cirrus Jet uses a Cirrus EXP5000 primary flight display. Figure 37 shows the instrument panel of the Cirrus Jet with labels on the commonly used instruments. 


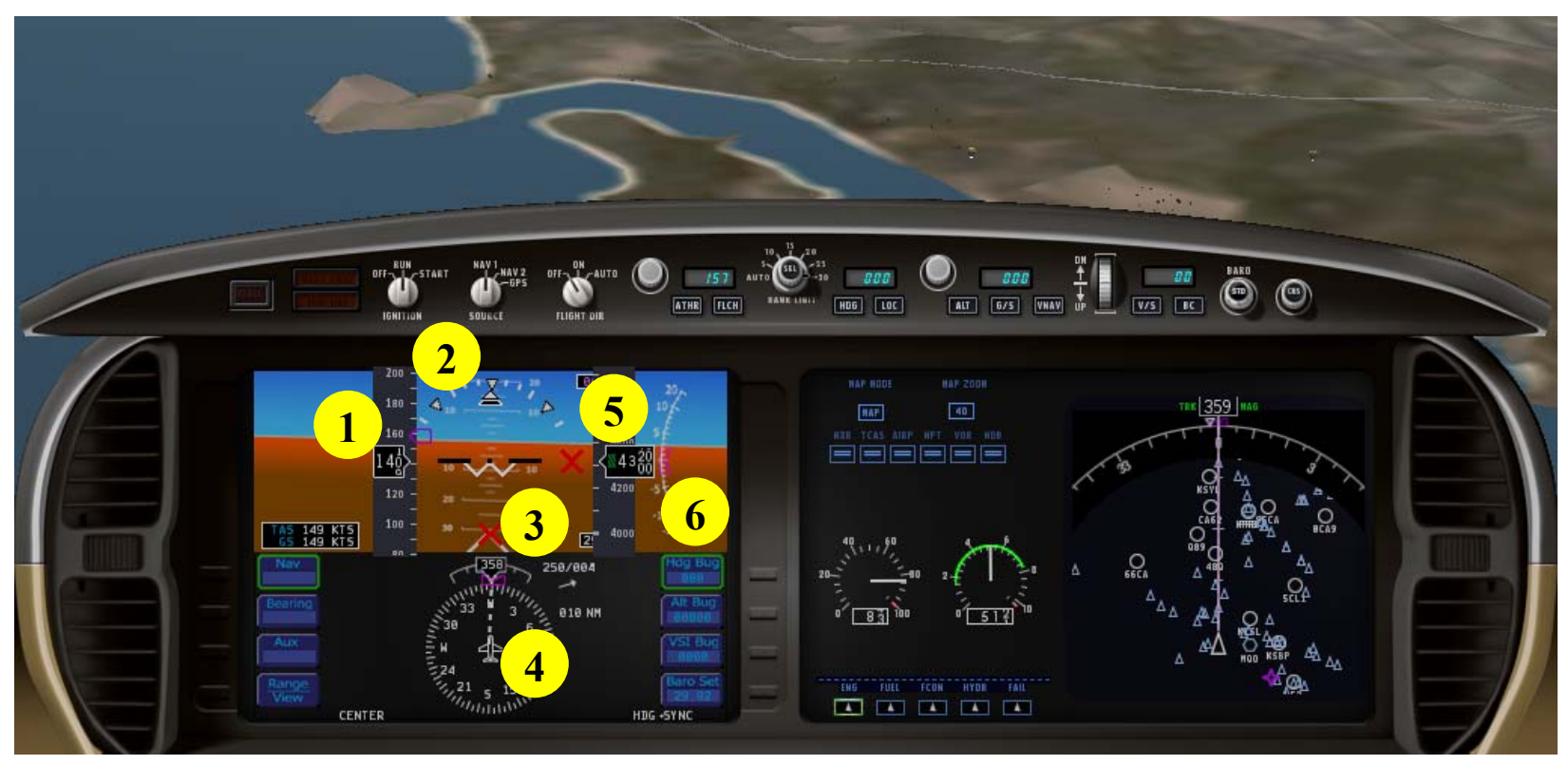

Figure 37 - Cirrus instrument panel. (1) Airspeed indicator: Knots (2) Bank angle indicator: degrees (3) Pitch ladder: degrees (4) Magnetic Headings indicator (5) Altimeter: feet

(6) Vertical Airspeed indicator: feet

The instruments used in this experiment are the airspeed indicator, the bank angle indicator, the pitch ladder, the magnetic heading indicator, and the altimeter. The airspeed indictor measures the speed of the aircraft in knots. The bank angle indicator graduations are at $0,10,20,30,40,45$, and 60 degrees. The bank angle indicator measures the roll of the aircraft. The pitch ladder measures the pitch angle of the aircraft in degrees, taken with respect to the horizontal on the indicator. The magnetic heading indictor shows the direction the front of the aircraft is pointing. This does not show the true heading of the flight path. The altimeter measures the altitude of the aircraft in feet. The first two tests, VS-A and VS-C, are flight training tasks used to teach the subject to use instruments on the panel. The final test provides a task with the most amount of visual sensory information as the subject focuses on the chase aircraft rather than the instrument panel. 


\subsection{X-Plane Computer}

The X-Plane computer runs the actual flight simulator. The computer must have the X-Plane Flight Simulator software installed, and the simulator must be configured to properly transmit information to the computer running the GVS Software. Figure 38 shows the window for entering a port and the IP address of the destination computer.

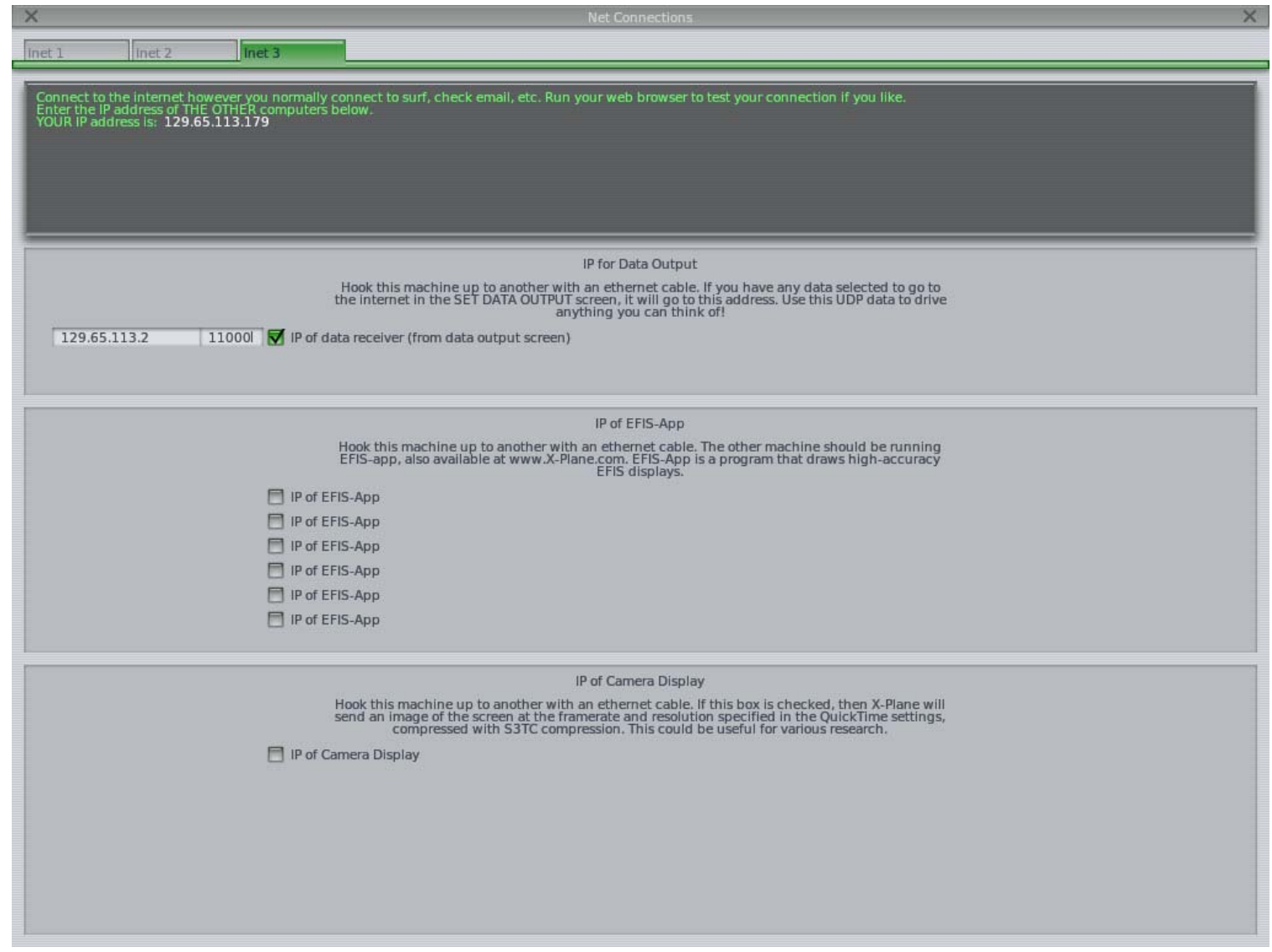

Figure 38 - X-Plane window for entering in the IP and port for Data Output

In addition to making sure X-Plane sends information to the correct location, it is also important to make sure the correct information is being sent. Figure 39 
shows the X-Plane Data Input \& Output window for selecting what information to include in the datastream.

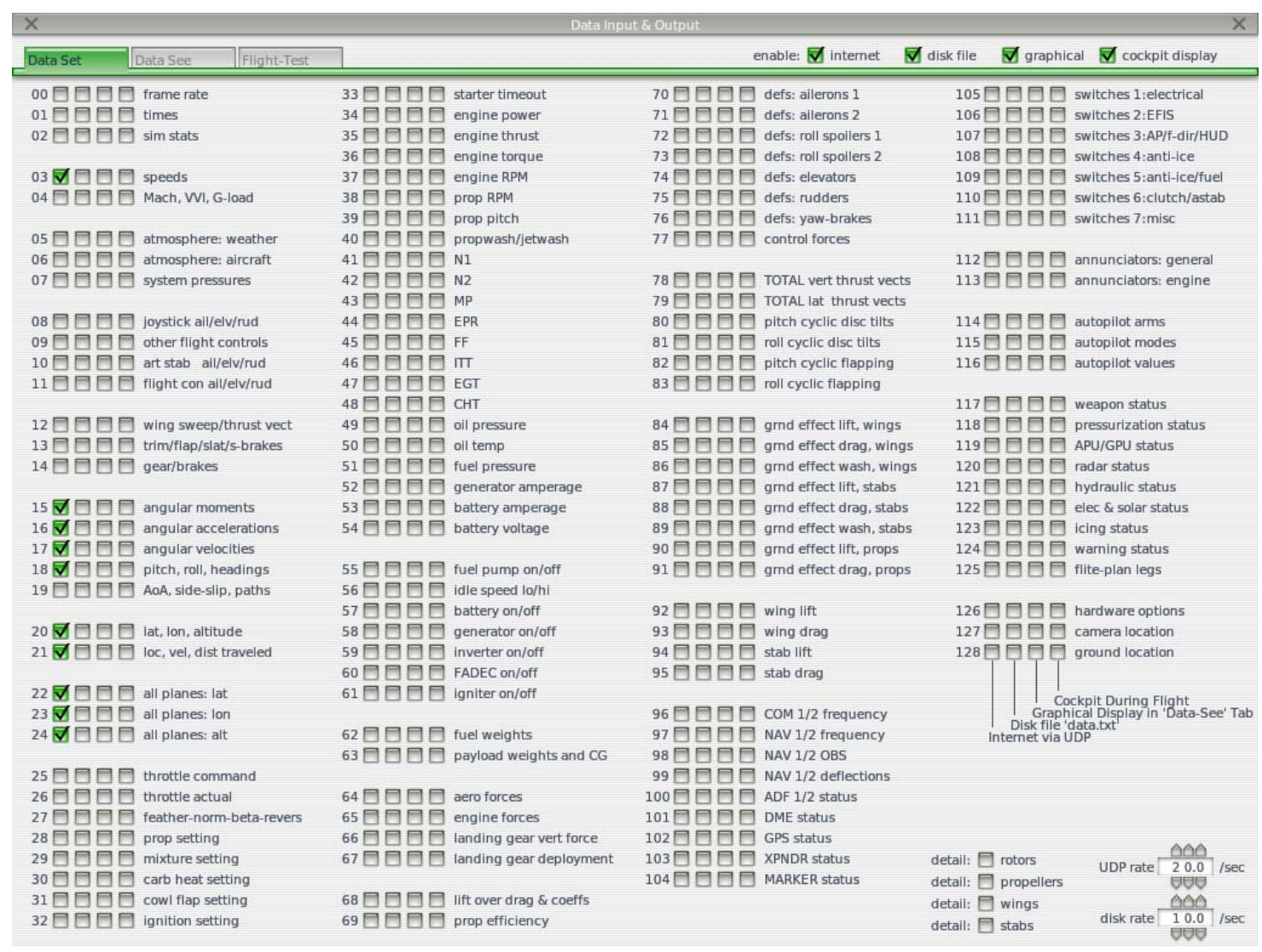

Figure 39 - X-Plane selected data output: speeds, angular moments, angular accelerations, angular velocities, pitch, roll, headings, latitude, longitude, altitude, location, velocity, and distance traveled.

For the tests run in this experiment, the standard X-Plane output data are: speeds, angular moments, angular accelerations, angular velocities, pitch, roll, headings, latitude, longitude, altitude, location, velocity, and distance traveled. The bottom right hand of the GUI in Figure 39 also lets you indicate how many data points you want X-Plane to output per second. The default UDP transfer rate is 20 data points per second, which is what was used for this experiment. The four major columns in Figure 39 each contain three basic pieces of information: data 
number, output location, and a brief description. For example, \#18 includes output to the Ethernet port (the $2^{\text {nd }}$ checkbox denotes a text file, $3^{\text {rd }}$ a graph in $\mathrm{X}$ Plane, and the $4^{\text {th }}$ a graph to the cockpit). In order to have the GUI record data the Ethernet or left most checkbox must be selected. All data with a check next to the left box, the Ethernet Port, transmits to the GVS Software.

X-Plane must also be configured to connect to the computer running the chase plane replay. The computers are connected through the multiplayer functionality of X-Plane. Figure 40 shows the setup box for the multiplayer settings.

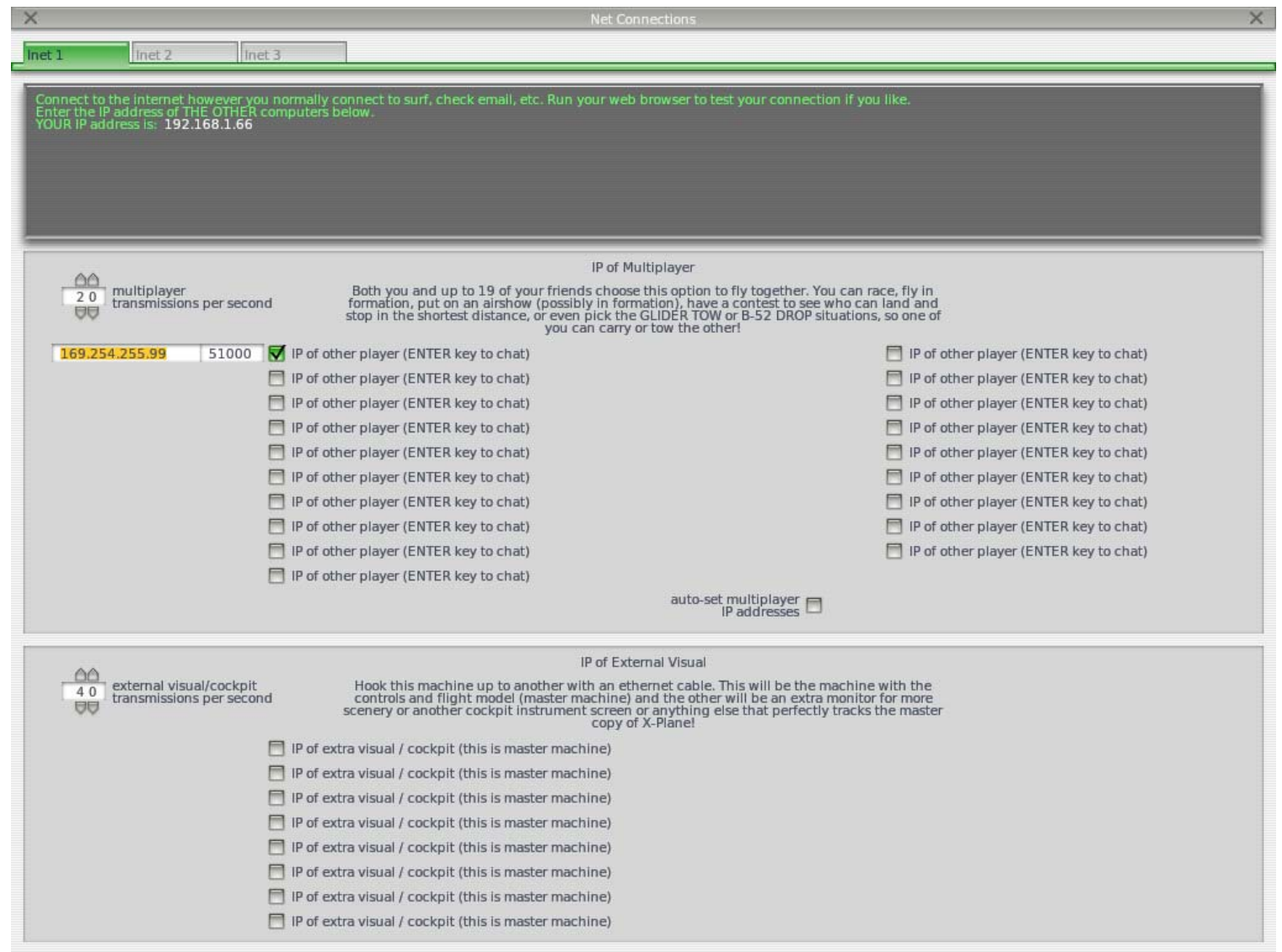

Figure 40 - X-Plane Multiplayer Setup 
In order to connect to another X-Plane computer the IP address of the other player must be entered. In addition a port number must be selected for the Ethernet connection. The Chase plane computer must be configured with the IP address of the subject's X-Plane computer.

\subsection{X-Plane Chase Plane Computer}

The final device in the X-Plane system design is the Chase Plane Computer. In order to setup a chase plane a flight simulation must be saved. Figure 41 shows the dialog box for saving a flight simulation.

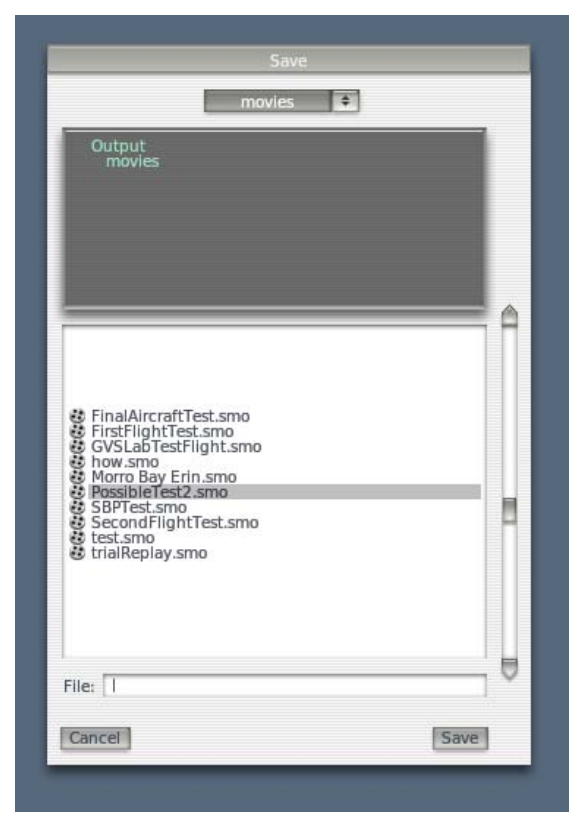

Figure 41 - Saving a flight simulation for the Chase Plane

The recorded flight path provides a repeatable simulation which can be used as the chase plane. When you link the computer playing the recorded flight path with the test subject's computer, the recorded aircraft flight shows up in their simulation environment as the chase plane. The chase plane can be played at 
various speeds including backwards. Figure 42 shows a sample screen shot of a recorded flight including the various play modes.

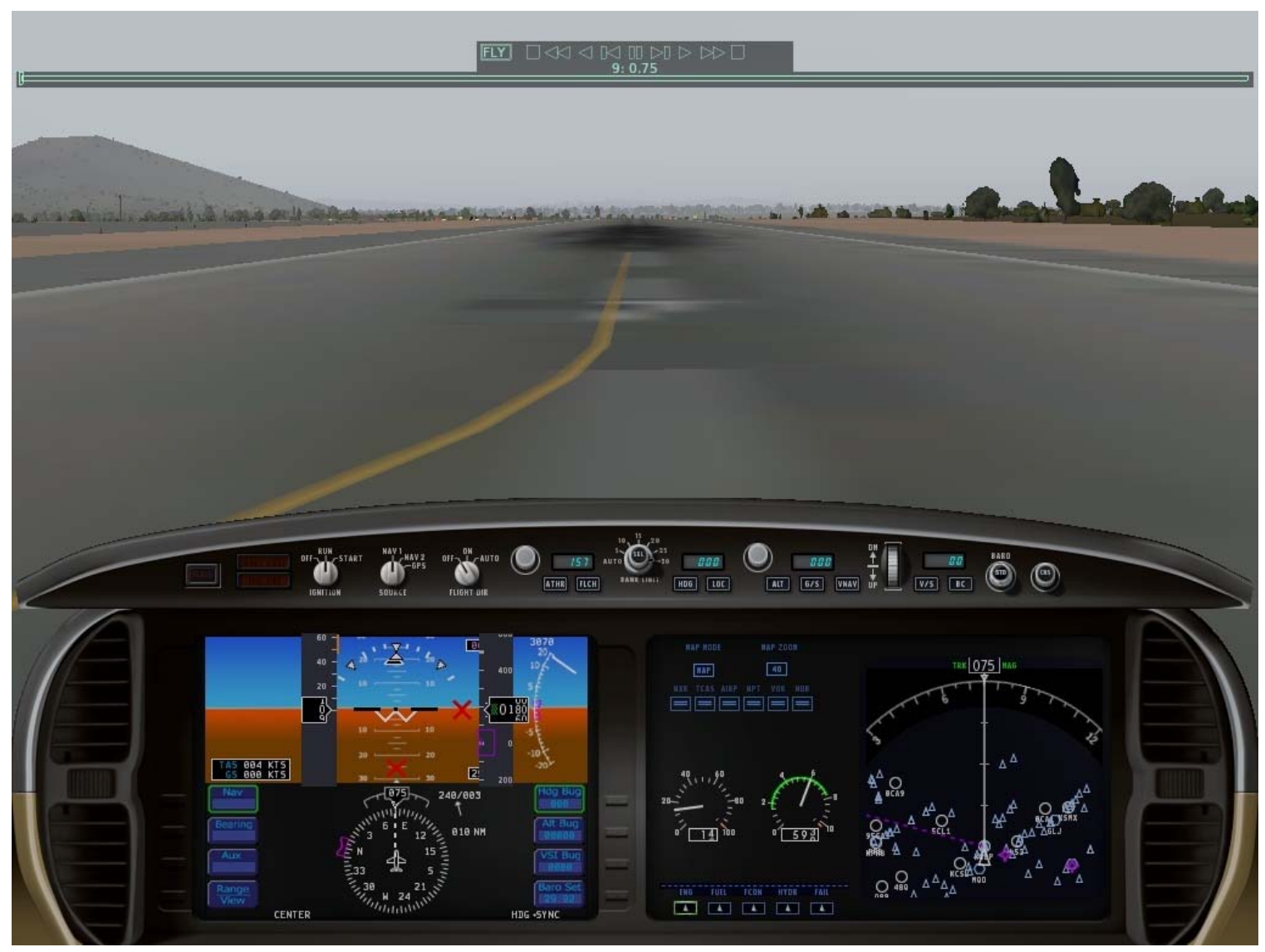

Figure 42 - Recorded flight screen 


\section{CHAPTER 5 : Experimental Protocol}

The system design and the experimental procedures were approved by the $\mathrm{Cal}$ Poly human subjects committee. This chapter explains the steps taken to safely test GVS on human subjects. Before any experimental procedures were performed, informed consent was received from the subject. All procedures were explained to the participant, and any questions or concerns the participant had addressed. When the subject was ready, they signed the informed consent document. The subject then filled out The Motion Sickness Screening Questionnaire (MSQ)[12], which provides insight to their vestibular history. The participant was asked to indicate any reason they should not participate such as history of vestibular disorder, epilepsy, or any neurological or musculo-skeletal problems [1]. If there were any doubts as to whether the subject should participate, the experimenter refused to test the subject. It should be noted that the GVS coupled flight simulator tests performed in section 5.3 did not include the pitch stimulations. The configuration used at the mayo clinic does not generate significant pitch sensations in our system. Each test in the experiment is performed with roll stimulations only.

\subsection{Initial setup procedures (25 minutes)}

- Explain procedures and obtain informed consent (5 minutes).

- Have the subject fill out a MSQ (5 minutes).

- Ensure they pass all exclusionary criteria (2 minutes). 
- Connect the subject to the GVS, which stimulates sensations of yaw, pitch and roll (5 minutes).

o Clean electrode sites shown in Figure 5 by rubbing them with an alcohol prep pad.

o Prepare the site by scrubbing the Nuprep skin prep on top of the cleaned site with a Q-tip. Apply the gel with one side of the Q-tip and gently abrade the surface of the skin by stroking the other side of the Q-tip until the gel has almost dried

o Place the electrode on the prepared area.

- Repeat for Electrode 1, Electrode 3, and the COM Electrode sites shown in Figure 5 making sure to use the GND electrode for the COM site on the back of the neck.

- Measure and record the resistance from Electrode 1 to COM and Electrode 3 to COM. If the resistance exceeds $10 \mathrm{~K} \Omega$ clean the site again. Scrub the site with Nuprep skin prep and a Q-tip. Replace the electrode and measure the resistance again. Repeat until resistance is below $10 \mathrm{~K} \Omega$ (5 minutes).

- A pretest is administered to ensure comfort of the subject and proper function of the device. The pretest consists of a roll right or a roll left stimulation with manual ramping of the max current from zero to the maximum current level used in the stimulation, not to exceed 1.5 milliamps. The pretest is manually administered in steps of no more than 0.25 milliamps beginning at 0.2 milliamps. Subjects remain seated during the pretest. The subject is asked to comment on the sensation of current entering the body. The current level is increased with the 
permission of the subject with the intent to exceed $1.5 \mathrm{~mA}$. The test is not administered if the subject refuses to exceed $0.5 \mathrm{~mA}$, which is a commonly used threshold for orientation sensations. After an acceptable current level has been agreed upon the tests may be administered to the subject (3 minutes).

\subsection{Motion Sensitivity Test (45 minutes)}

- Perform the initial setup procedures from section 5.1 (25 minutes).

- The motion sensitivity test should be administered in the dark. The subject should have their eyes closed and their feet together while standing (5 minutes)

o Select the "Begin Test" button located on the bottom right of the GVS GUI. The automatically programmed motion sensitivity test begins.

o After every two rocking sensations ask the subject on a scale of 0-6 how they feel in terms of motion sickness. (0: no symptoms; 1 : any symptoms, however slight; 2: mild symptoms, e.g., stomach awareness but not nausea; 3 : mild nausea; 4: mild to moderate nausea; 5: moderate nausea but can continue; 6: moderate nausea and want to stop) [13]. Two rocking sensations correspond to 16 commands being sent, which populate the messages sent text box of the GVS GUI.

o Document the responses of the subject on the Simulator Sickness Test Administrator sheet.

o After the $5^{\text {th }}$ rocking sequence, allow the rocking to continue for 30 seconds or until the subject requests to stop. Stop the testing by selecting the "Stop Button" located on the bottom left hand corner of the GVS GUI. 
o Record the subject's final orientation sensation.

o Record any comments from the subject or administrator of notable activities.

- Perform Final Procedures from section 5.4 (15 minutes).

\subsection{GVS coupled flight simulator (90 minutes)}

- Initial Setup Procedures from section 5.1 (25 minutes)

- Training (25 minutes) - The test administrator should enter in the test number for the filename and the desired folder location to save the files.

o The aircraft controls and joystick functionality are explained to the subject.

o The VS-A test is explained and practiced by the subject.

o Test1: The VS-A complete test is performed and recorded.

o The VS-C test is explained and practiced by the subject.

o Test2: The VS-C complete test is performed and recorded.

o Test3: The chase task is completed and recorded.

- Measurement Technique (35 minutes) - The test administrator should enter in the desired max current, the subject number, and select trial 1, 2, or 3 on the GUI. Then use the congruent, conflicting, and sham test buttons on the GUI.

o Select Trial 1 on the GUI. Have the subject complete the VS-A task with sham, congruent, and conflicting stimulations administered in a random order. 
o Select Trial 2 on the GUI. Have the subject complete the VS-C task with the congruent, conflicting, and sham stimulations administered in a random order.

o Select Trial 3 on the GUI. Have the subject complete the chase plane task with conflicting, sham, and congruent stimulations administered in a random order.

- Perform Final Procedures from 5.4 (15 minutes).

\subsection{Final procedures (15 Minutes)}

- Remove and dispose of electrodes (2 Minutes).

- The subject is asked to fill out the Simulator Sickness Questionnaire (8 minutes).

- The following three standardized field sobriety tests as described by the National Highway Safety Traffic Administration[23] are performed as a cautionary measure to ensure safe vestibular function (5 minutes)

o The horizontal gaze nystagmus

o The walk-and-turn

o The one-leg stand.

- There is a 24 hour follow-up phone call to ensure vestibular function is normal. 


\section{CHAPTER 6 : Data and Analysis}

This chapter reports the data obtained from the Motion Sensitivity experiment and the X-Plane Flight experiment. Each of the tests performed in X-Plane Flight experiment report the performance of each flight task based on RMS deviations from the desired value in response to congruent, conflicting, and sham stimulations. This chapter displays the deviations and reports the statistical relevance of the deviations of the different stimulations for the VS-A, VS-C, and Chase Plane Test. For each of the tests, a one-way repeated measures analysis of variance (ANOVA) with a significance level of 0.05 was conducted.

\subsection{Motion Sensitivity Test}

All subjects in the motion sensitivity test reported sensations of rocking back and forth. Figure 43 shows the sickness scores for each of the subjects at the different rocking frequencies. Each color in the plot represents a score for a subject. The blue bar in the plot shows the average of all the scores at each rocking frequency and the linear trend of the average. 


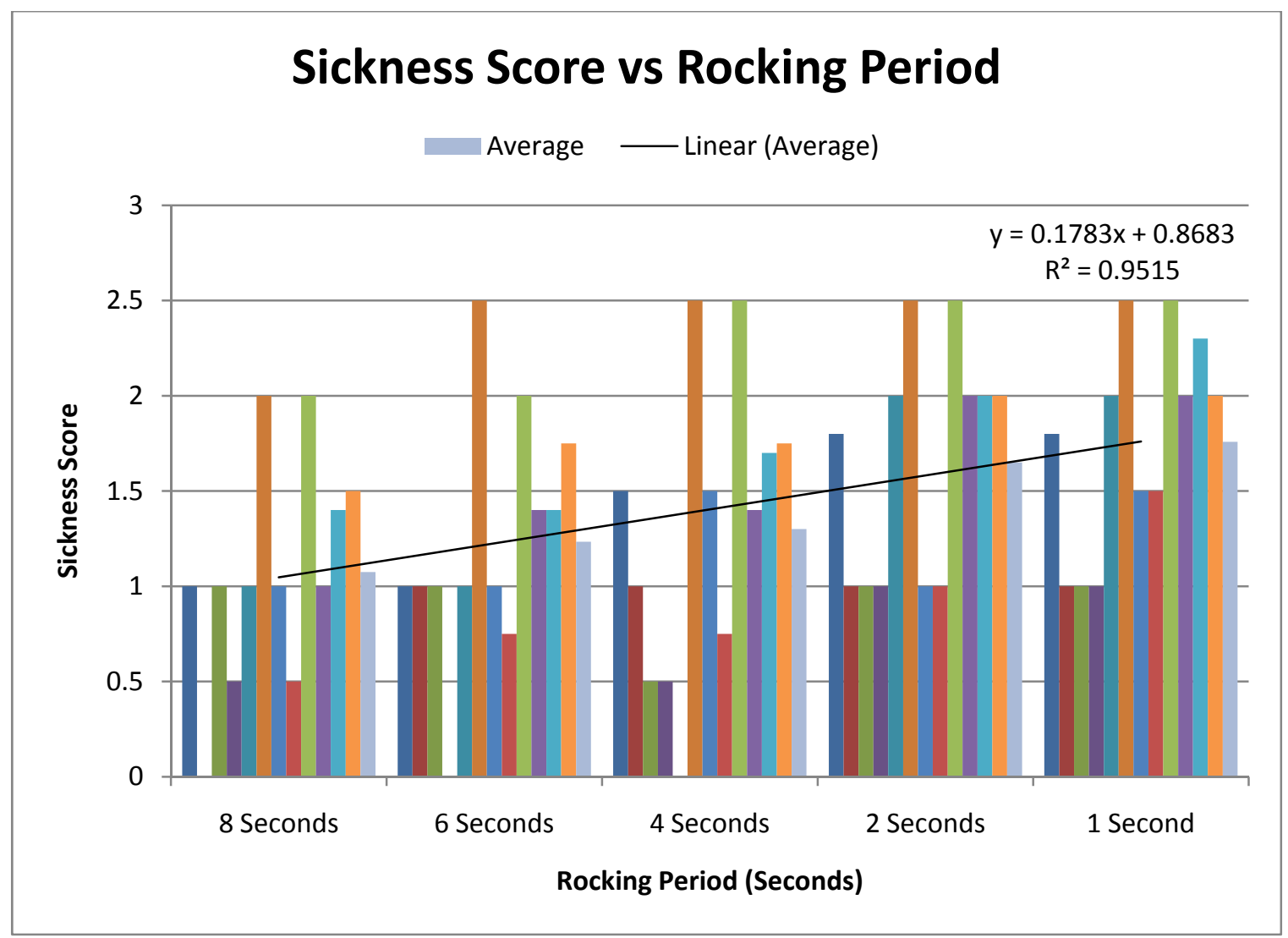

Figure 43 - Motion Sickness Score associated with the varying rocking frequency tests

The linear fit line indicates the Sickness Score increases as the rocking frequency increases. Figure 44 shows the final sickness score reported after the test versus the total motion sickness score generated from the Motion Sickness Questionnaire. 


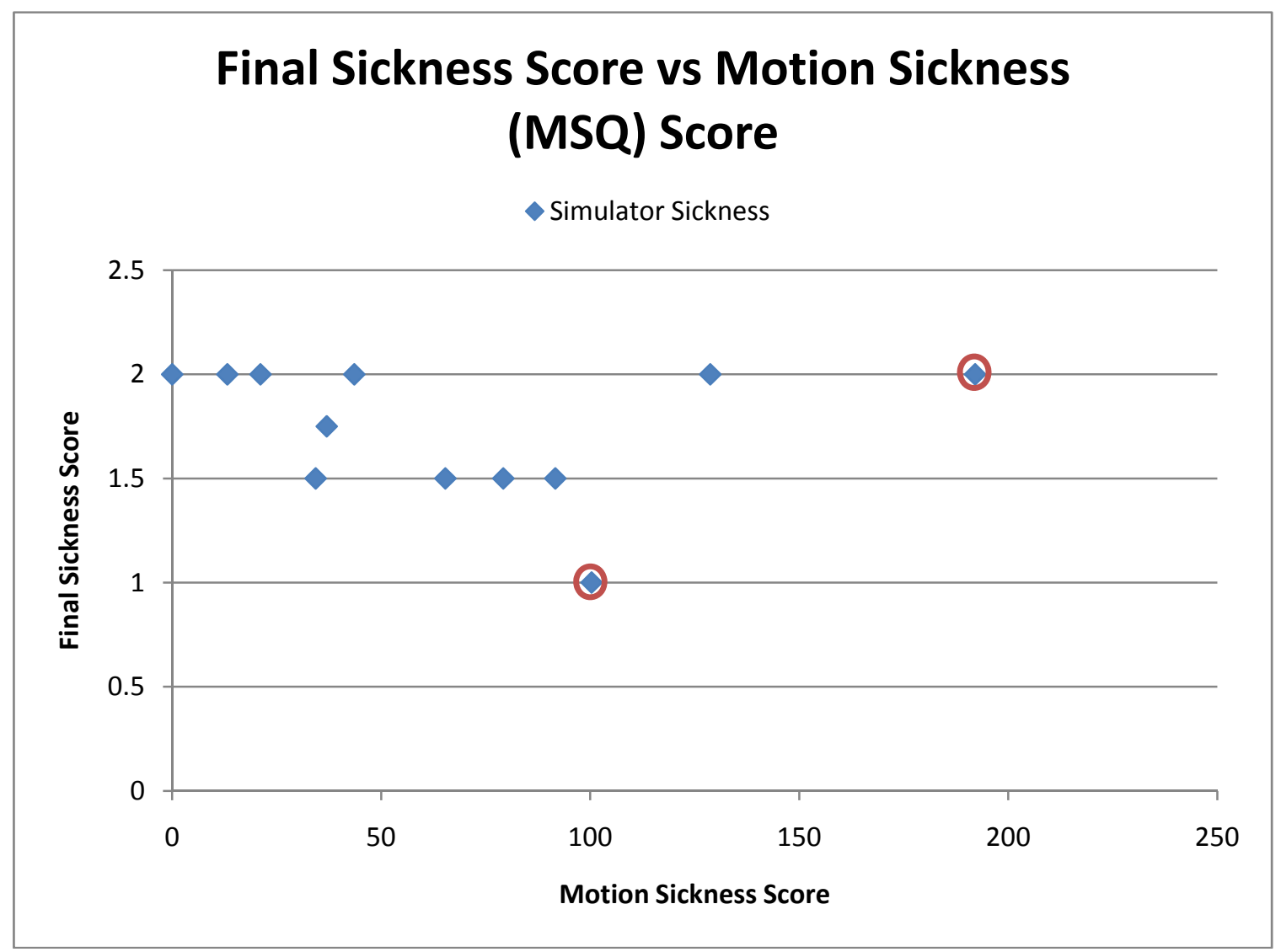

Figure 44 - Final Sickness scores of the subjects versus the motion sickness scores of the subjects

Figure 44 also includes two red circles around data points on the plot. These two subjects both reported feeling nausea within 15 minutes after testing had completed. Both of these subjects also reported a metallic or battery acid taste in their mouths after testing. The metallic taste was also observed during the application of high currents in the function MRI study by Lobel et al.

\subsection{Flight Simulator Tests}

This section presents the flight simulator results. The VS-A, VS-C, and chase plane tests were performed with only roll stimulations. The pitch stimulation configuration used by the mayo clinic did not generate significant sensations in 
our system configuration. The roll stimulation configuration did stimulate roll sensations. The code for generating the pitch sensations is commented out during all of the tests.

\subsubsection{Test 1: VS-A}

Figure 45 shows one subject's paths during Test 1 (VS-A). The plot shows paths for completing the task during the pre-test, conflicting stimulation, and congruent stimulation. The task begins on the right side of the plot at the origin.

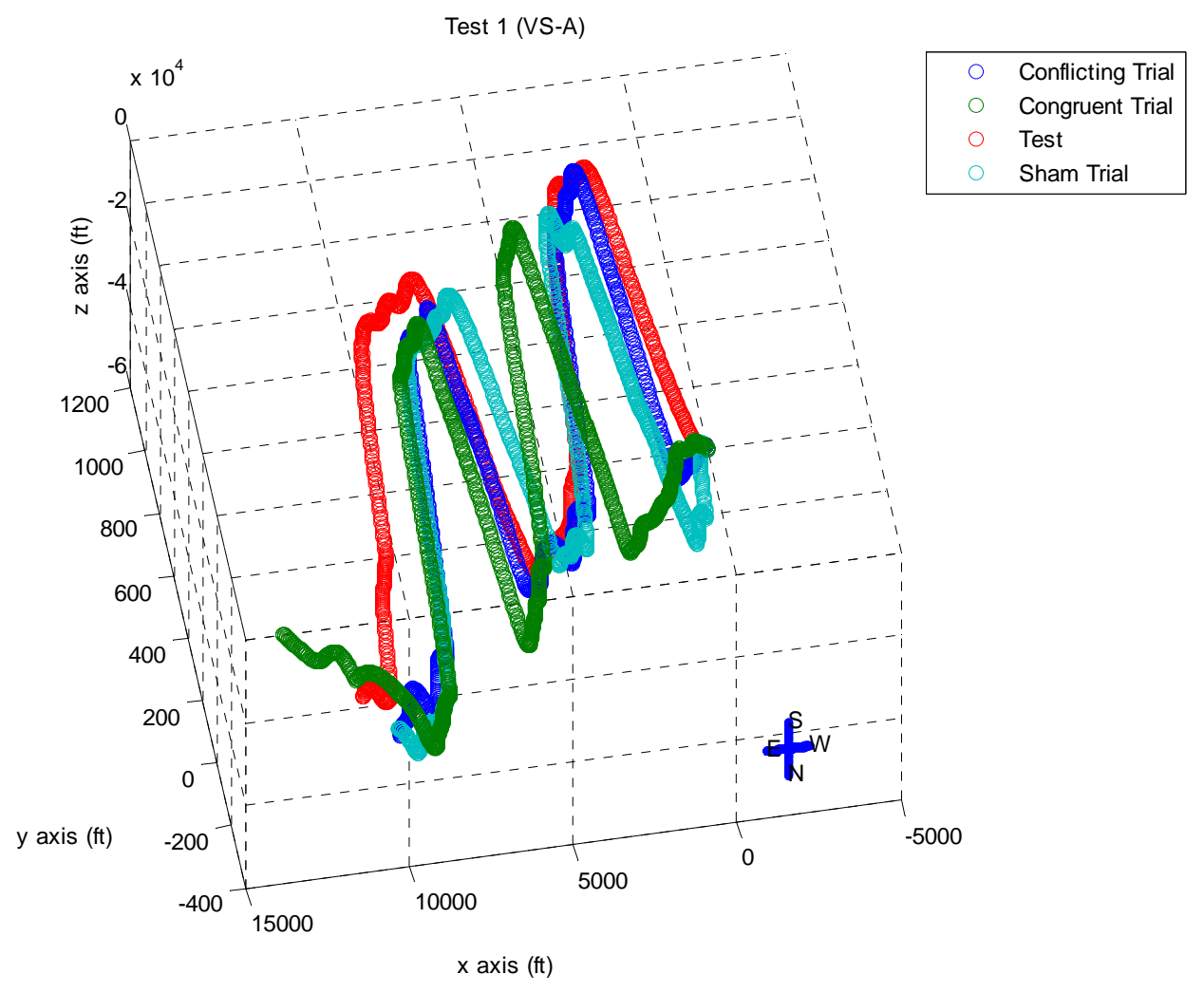

Figure 45 - Sample Test 1 (VS-A) X-Plane flight path graphed in Matlab. The Pre-Test flight path had no stimulation. The $X, Y$, and $Z$ axes are measured in meters

The heading measured in this experiment is magnetic north. If the aircraft is facing towards magnetic north but there are side winds; the aircraft moves north and can be moved east or west depending on the direction of the wind. The true 
heading provides the actual direction of the resultant created from the magnetic heading and the side wind, while the magnetic heading provides the direction the aircraft is facing. Figure 46 provides a two dimensional (XZ) view of the paths to make it easier to see the true heading positions for each test. Again, the true heading of the aircraft is not towards north, but the aircraft is facing as close to magnetic north as the subject can maintain.

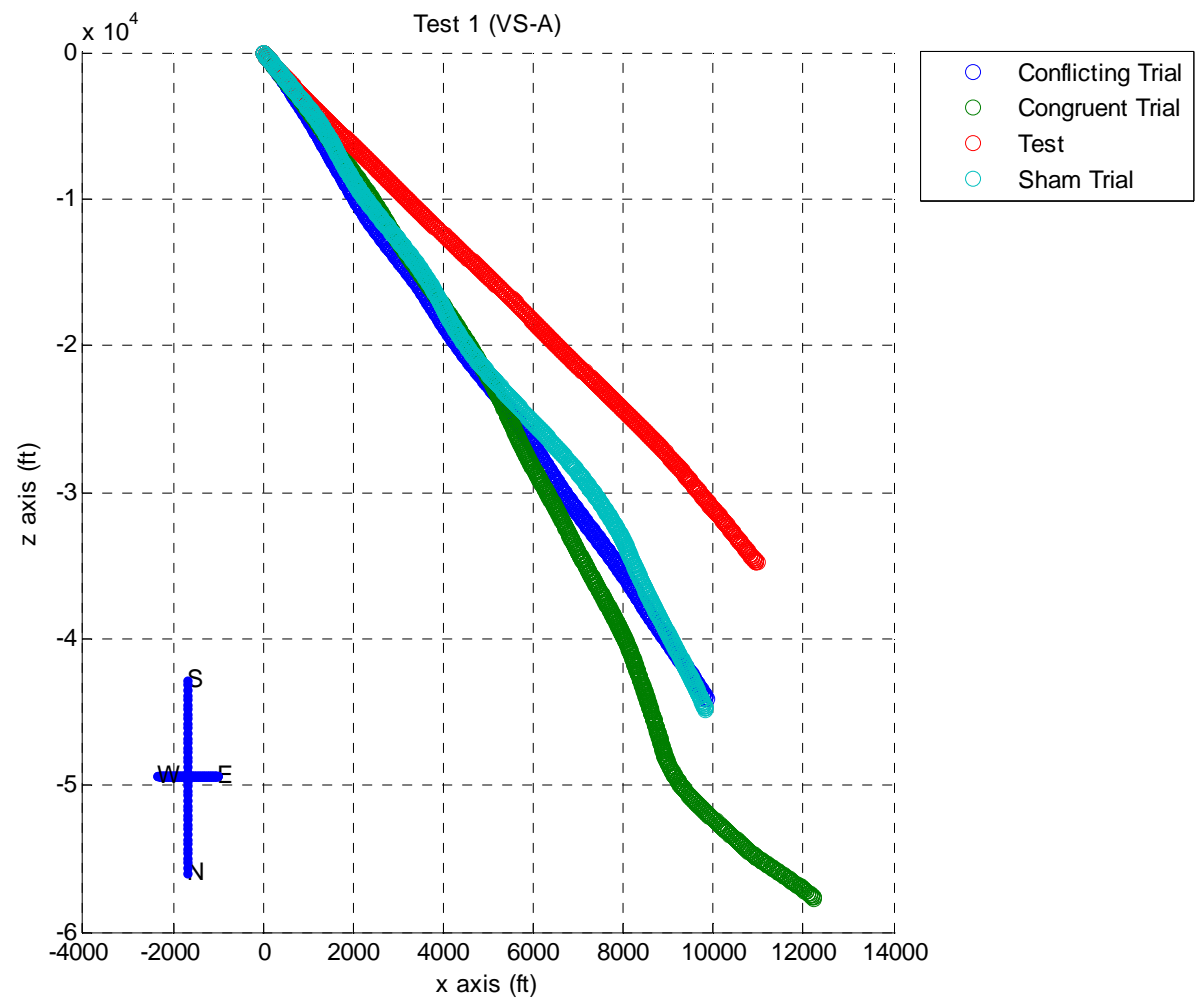

Figure 46 - Two dimensional (XZ) plot showing the aircraft true heading

As noted in the experimental procedures, the subject made two climbs and two descents, which can be seen in Figure 45. During each of these flight patterns, the subject was to maintain a 0 degrees magnetic north heading. Figure 47 shows the average magnetic headings of the subjects while completing these tasks with 
the different stimulation profiles. The blue bar in the plot represents the average heading for each stimulation profile.

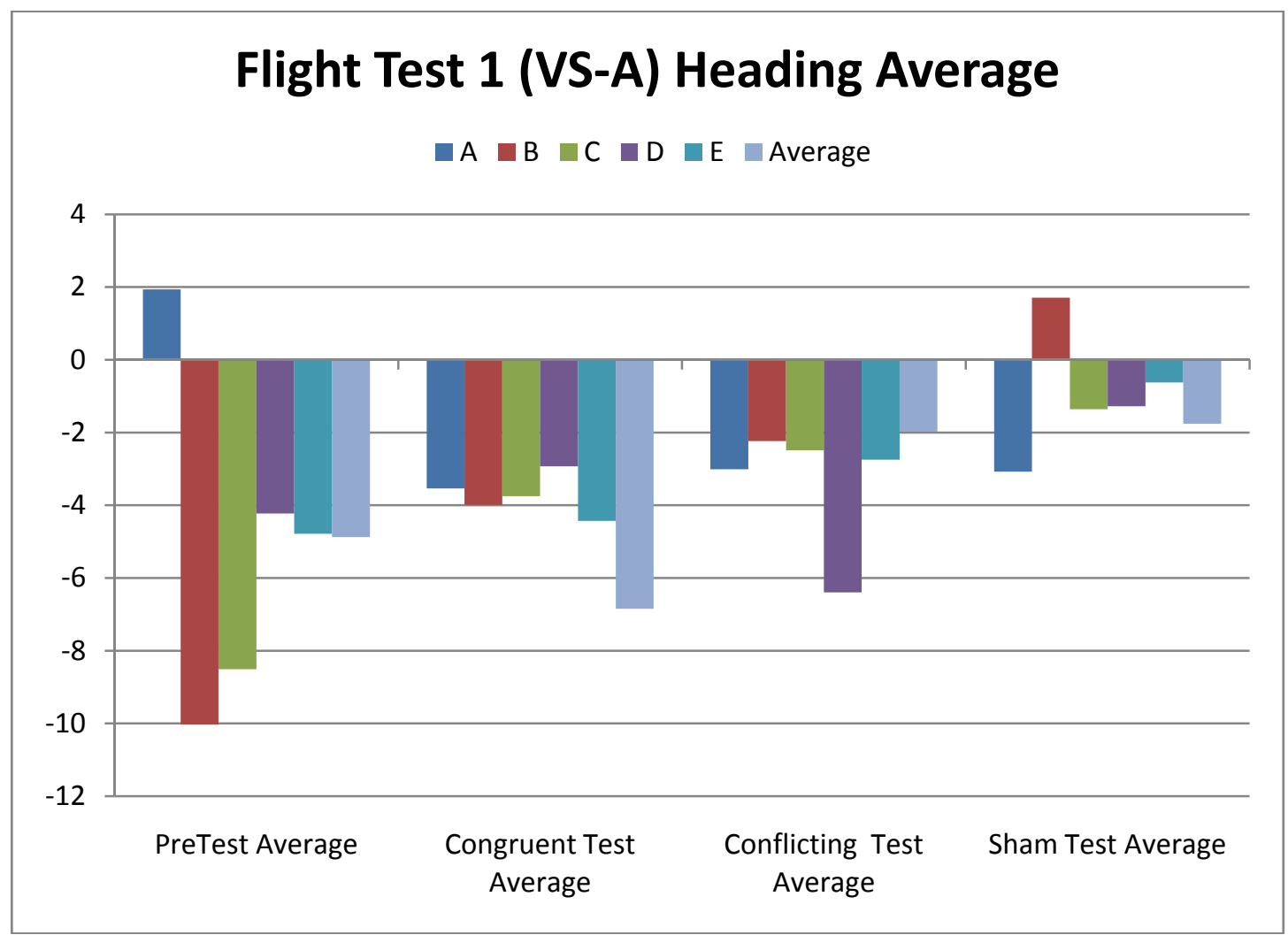

Figure 47 - Average heading values of each subject

It should be noted that if the subject completed the task correctly the aircraft would never roll past $+/-10$ degrees and no stimulations would take place. Many of the subjects were able to complete the task without feeling any stimulation. Root mean square (RMS) deviation was used to analyze the performance of the flight tasks. The formula used for the RMS calculations is:

$$
\theta_{\text {RMSdev }}=\sqrt{\frac{\sum\left(\theta_{\text {exp }}-\theta_{\text {ideal }}\right)^{2}}{n}}
$$


In this case $\theta_{\text {ideal }}$ is equal to 0 degrees and $\theta_{\text {exp }}$ is the magnetic heading of the aircraft maintained by the test subject. The $\mathrm{n}$ variable represents the number of samples. Figure 48 shows the RMS deviation from 0 degrees magnetic North for each of the stimulation profiles. The light blue bar in the plot represents the average deviation for each stimulation profile.

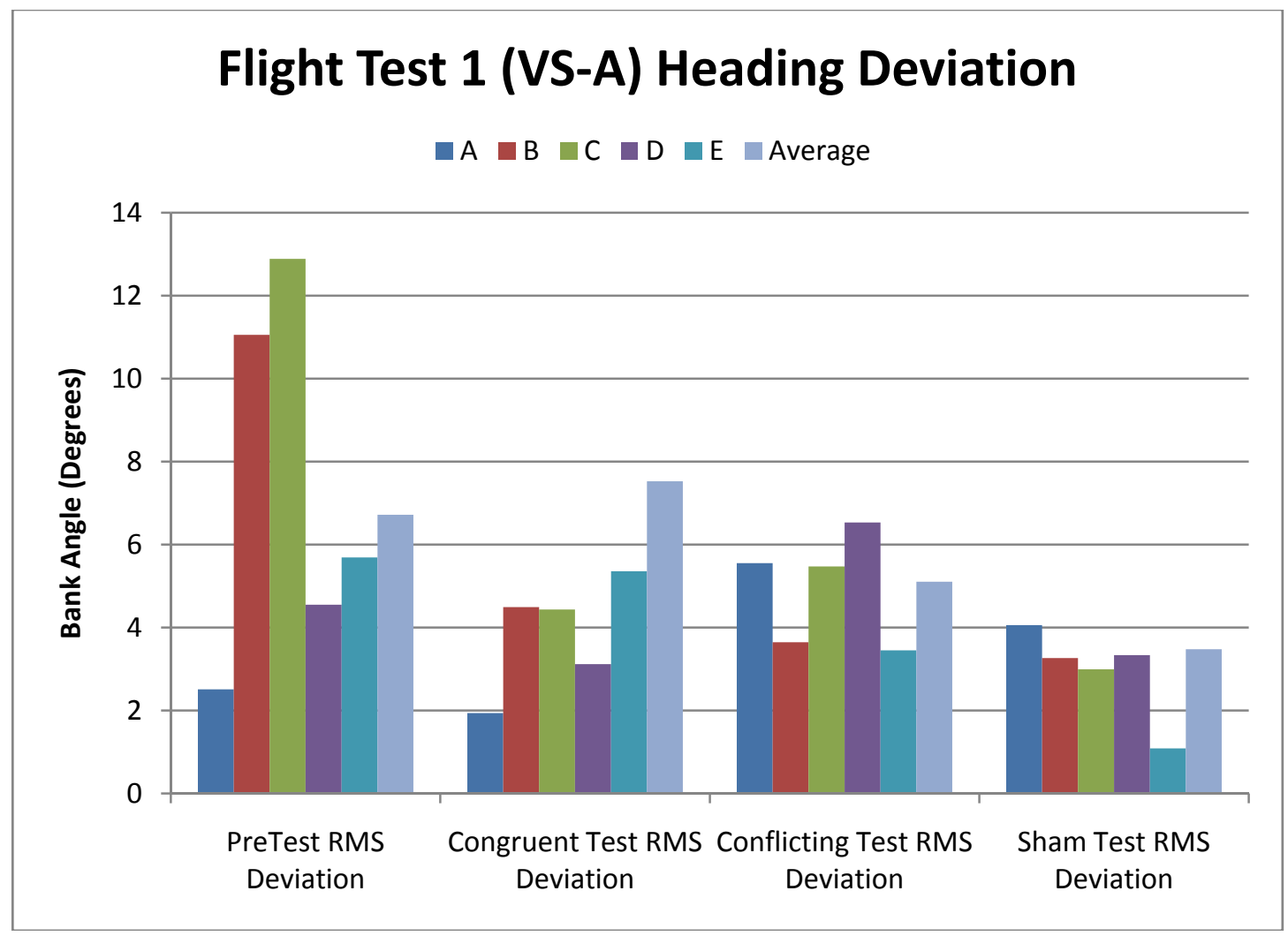

Figure 48 - Root mean square of the deviation of each subject's path from the desired 0 degrees North

The statistical analysis with the heading deviations as the dependent variable indicated that there is not enough statistical difference between the congruent, conflicting, and sham stimulations to exclude the possibility that the difference in deviation values for each stimulation profile is a result of random sampling variability $(\mathrm{P}=0.162)$. The data had a power of performed test with 
alpha $=0.203$, signifying negative findings could be a result of an insufficient sample size.

\subsubsection{Test 2: VS-C}

The second test profile with the X-Plane flight simulator was VS-C. This test required that the subject repeat the climbs and descents but this time with a +/-30 degree bank angle. Figure 49 shows a sample subject completing this task during the Pre-Test, congruent stimulation, conflicting stimulation, and the sham stimulation. The flight path begins on the right side of the plot at the origin.

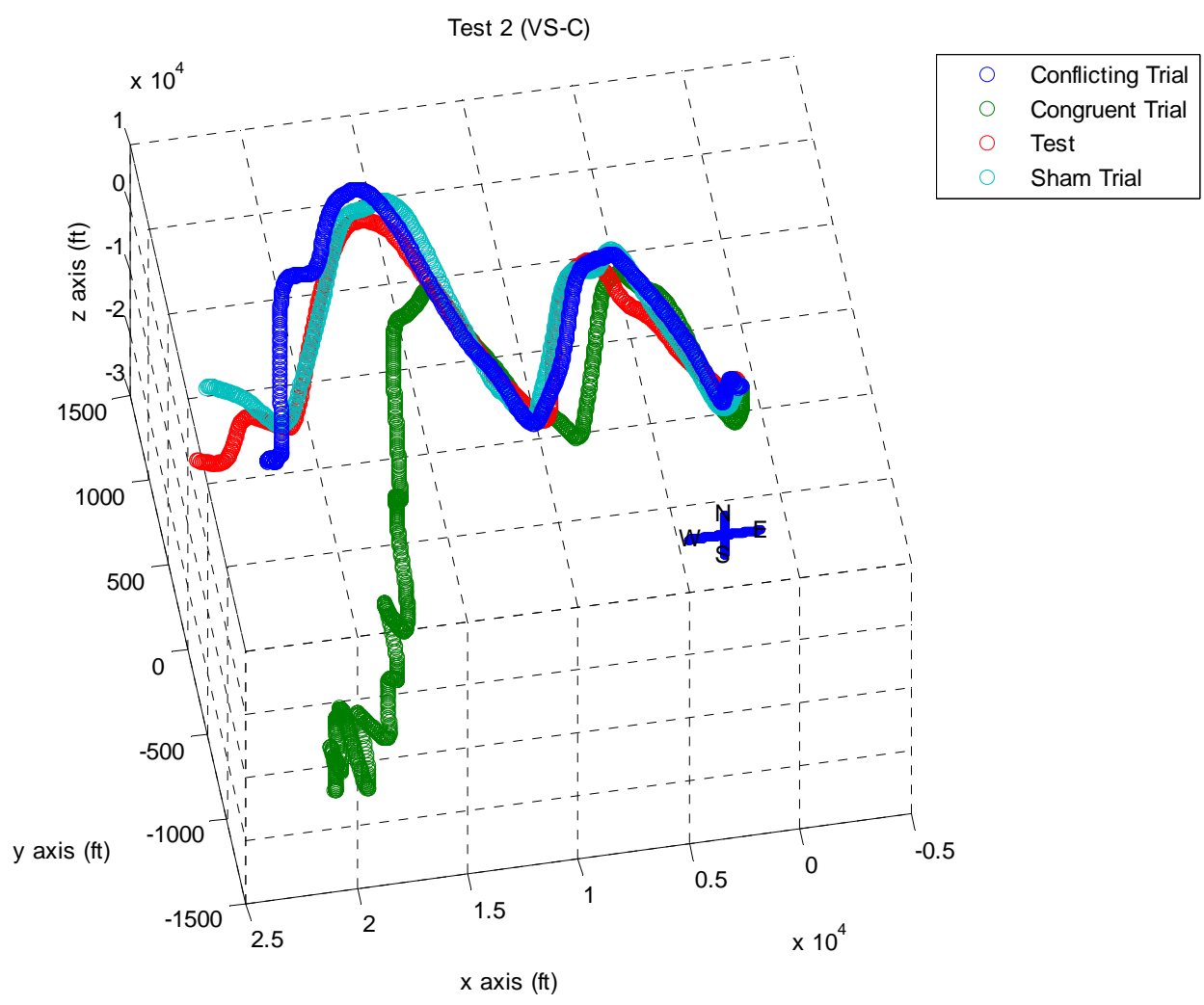

Figure 49 - Sample Test 2 (VS-C) X-Plane flight path graphed in Matlab. The Pre-Test flight path had no stimulation. The $X, Y$, and $Z$ axes are measured in meters. 
In this test the subject is stimulated during each climb and fall as a result of the bank angle. The altitude and bank angle are used to examine the performance of the subjects. The bank angle is examined between 4,250 feet and 4,750 feet, which ensures the subject has not begun to level off the bank and pitch of the aircraft. The RMS deviation from 30 degrees within the altitude boundaries provides a performance metric to compare between the stimulations. Figure 50 provides a representative altitude and roll plots to demonstrate which roll values are used in the RMS bank deviation calculation. 


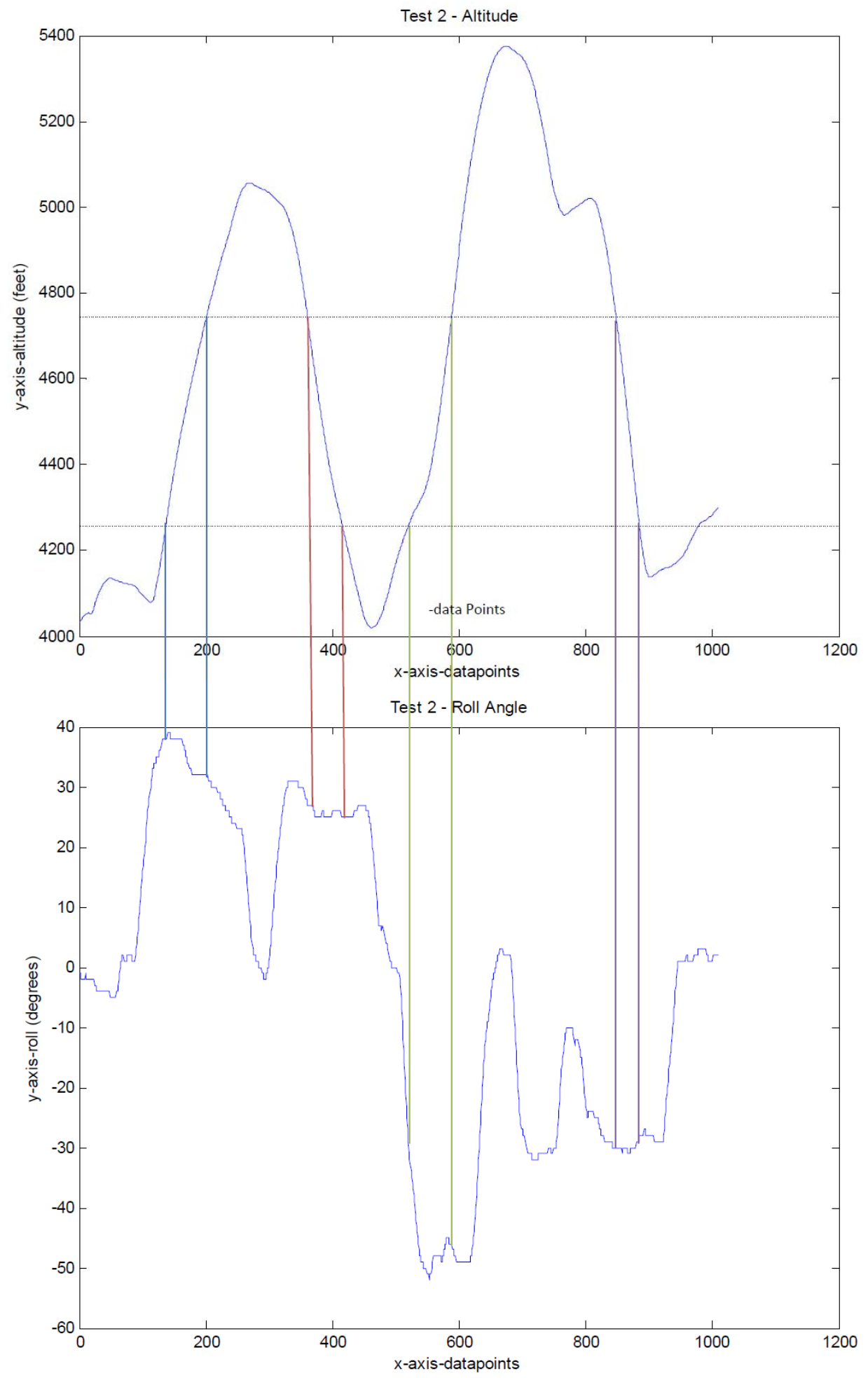

Figure 50 -Test 2 (VS-C) techniques for extracting roll values for the RMS roll deviation calculation. XPlane was set to output 20 data points/second. Every 200 points corresponds to 10 seconds. 
Figure 51 examines the subjects' performance during the stimulations. The data show the average bank angle and the RMS deviation from the desired +30 degree bank angle during the climb and descent between 4,250 feet and 4,750 feet. For each stimulation profile the average bank angle and the average RMS deviation are in light green.

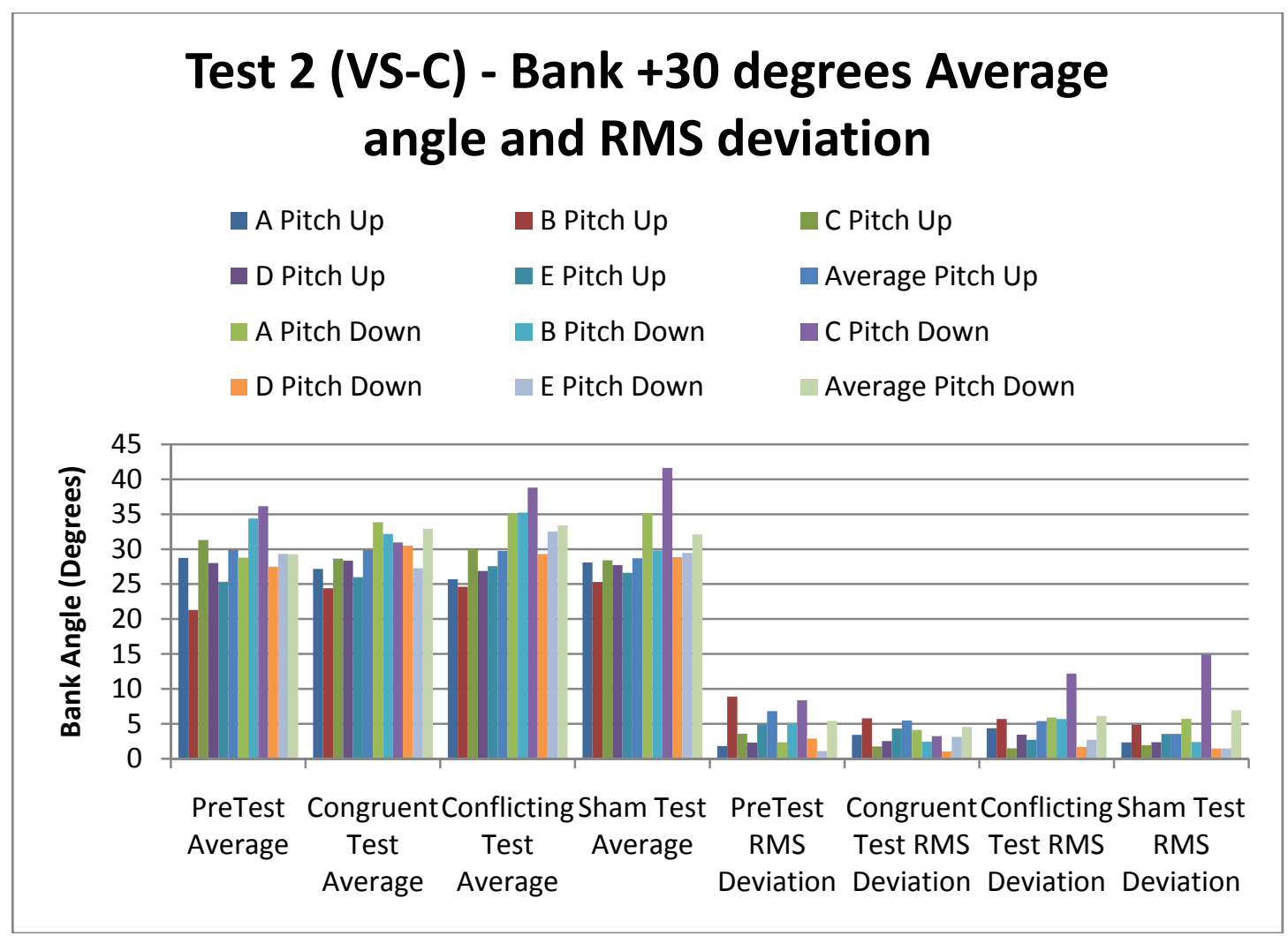

Figure 51 - Average bank angle and RMS deviation of Test 2 (VS-C) for +30 degree bank angle

The second part of the test required that the subject complete a climb and descend with a -30 degree bank angle. Figure 52 examines the performance of the subject during this task. This time the average bank angle and RMS deviation from the desired -30 degrees are plotted. The data was taken during the climb and descent between 4,250 feet and 4,750 feet. For each stimulation profile the average bank angle and the average RMS deviation are in light green. 


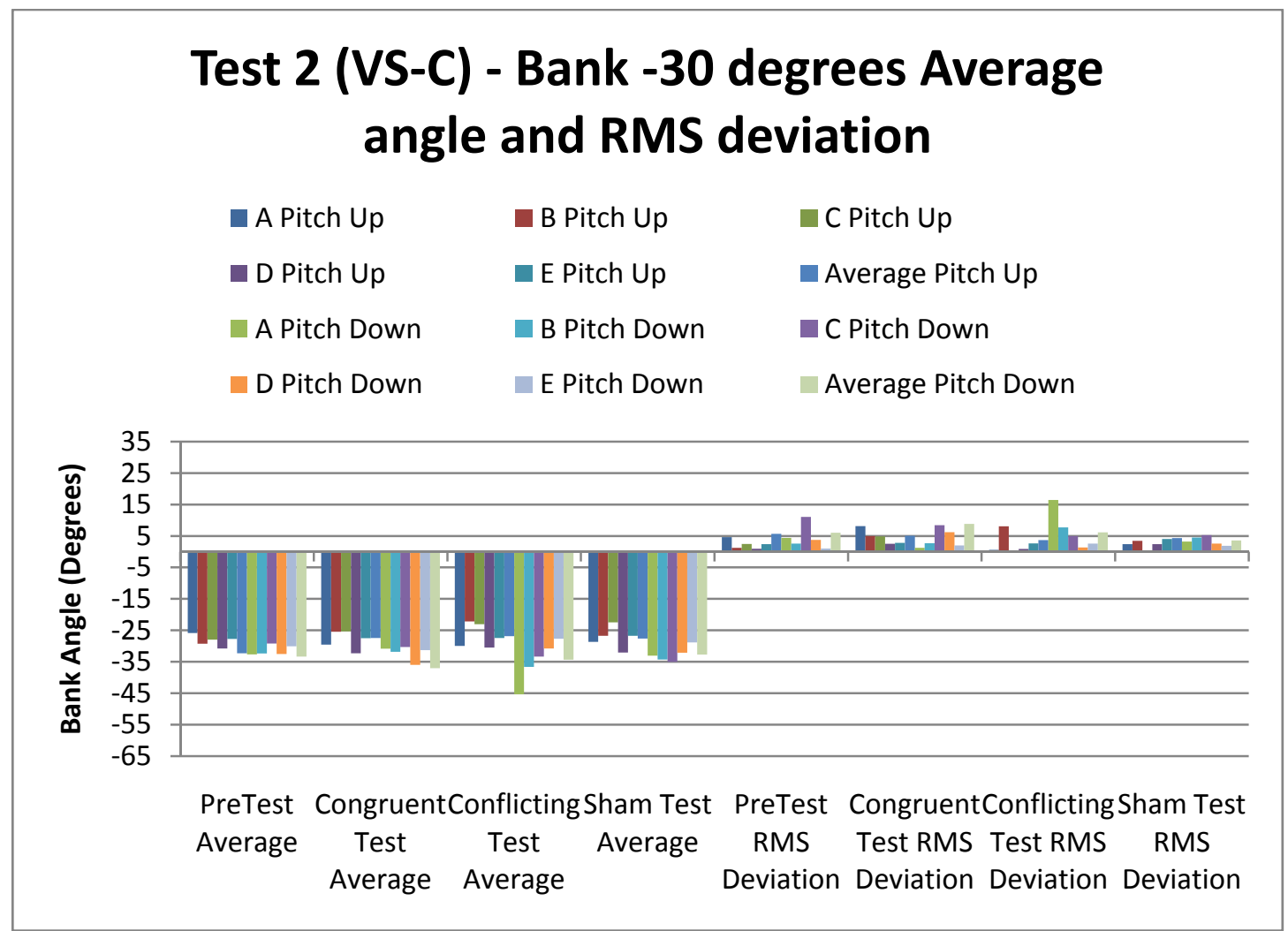

Figure 52 - Average bank angle and RMS deviation of Test 2 (VS-C) for -30 degree bank angle

Figure 53 shows the RMS deviation from the desired bank +/- 30 bank angles throughout the entire test. The data shows the deviations for the PreTest, congruent stimulations, confliction stimulations, and the sham stimulation. For each stimulation profile the average RMS deviation of the bank angles is plotted on the light blue bar. 


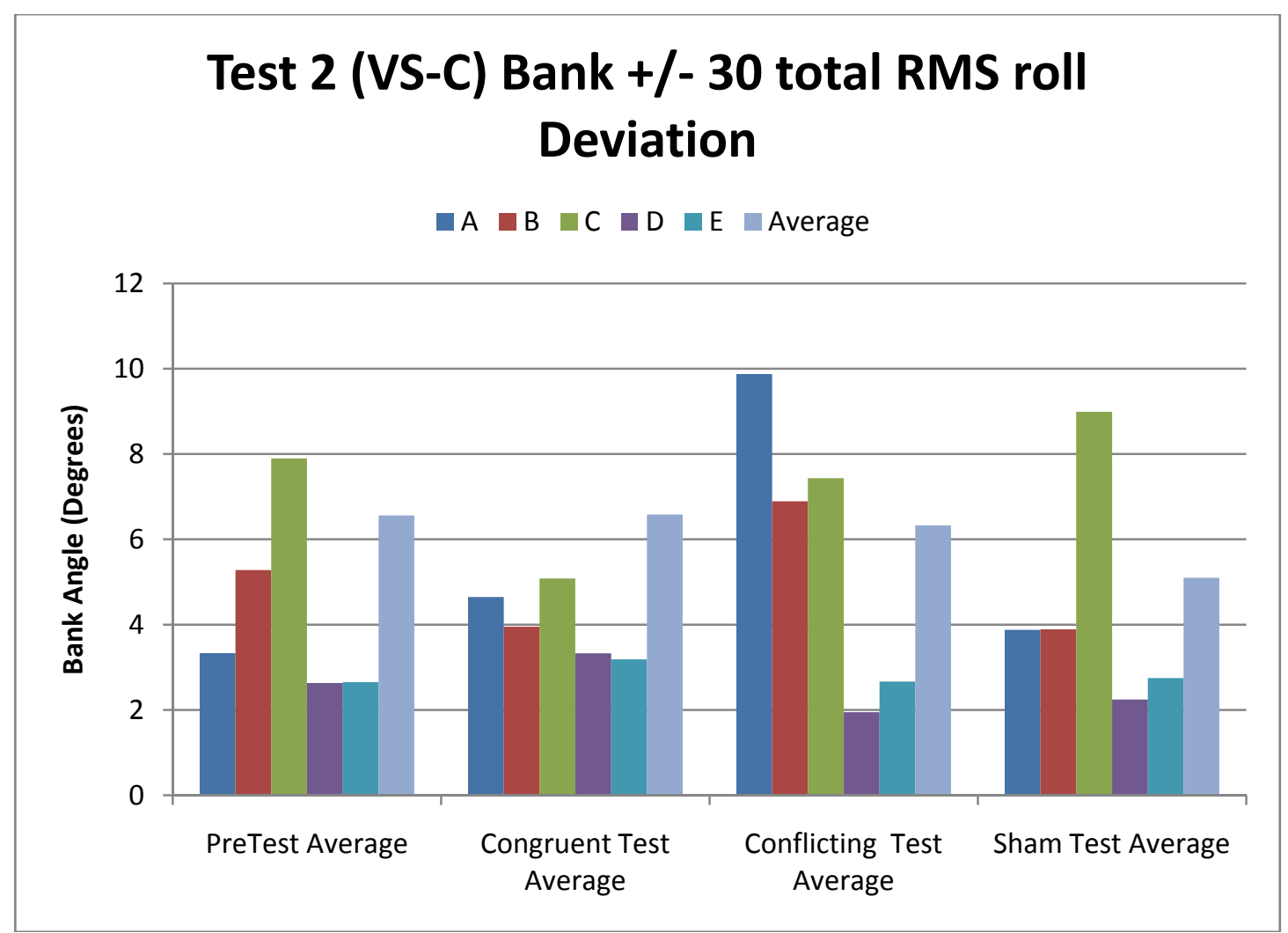

Figure 53 - RMS deviation of +/- 30 degree bank angle for Test 2 (VS-C)

The statistical analysis with total RMS roll deviation values of test 2 as the dependent variable indicated that there is not enough statistical difference between the congruent, conflicting, and sham stimulations to exclude the possibility that the difference in total RMS roll deviation values for each stimulation profile is a result of random sampling variability $(\mathrm{p}=0.346)$. The ANOVA had a power of 0.073 .

\subsubsection{Test 3: Chase Plane}

The first two tests were designed to have the subject use the instruments on the aircraft. The chase plane test forces the subject to remove their eyes from the instrument panel and follow a chase plane. Figure 54 shows the chase paths 
during various stimulation profiles. The chasing begins on the right side of the plot at the origin.

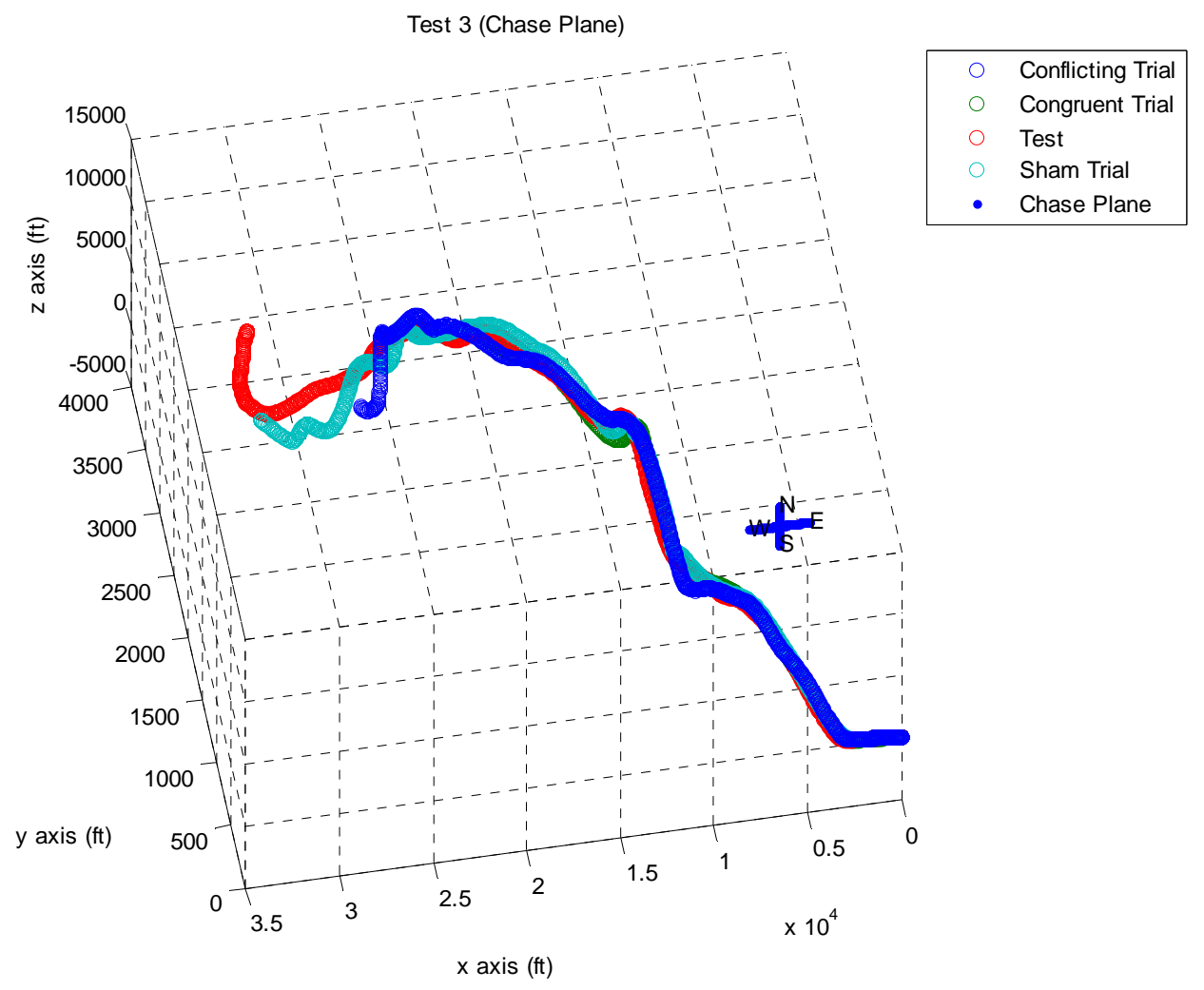

Figure 54 - Sample Test 3 (Chase Plane) X-Plane flight path graphed in Matlab. The Pre-Test flight path had no stimulation. The $X, Y$, and $Z$ axes are measured in meters.

Figure 55 shows a sample two-dimensional view of the flight pattern. This plot begins on the left side of the plot origin, viewing the flight path from beneath as if the viewer was lying on the ground. The paths look close to each other because of the large distances represented on the plot. Each of the X, Y, and Z axes are measured in meters. 


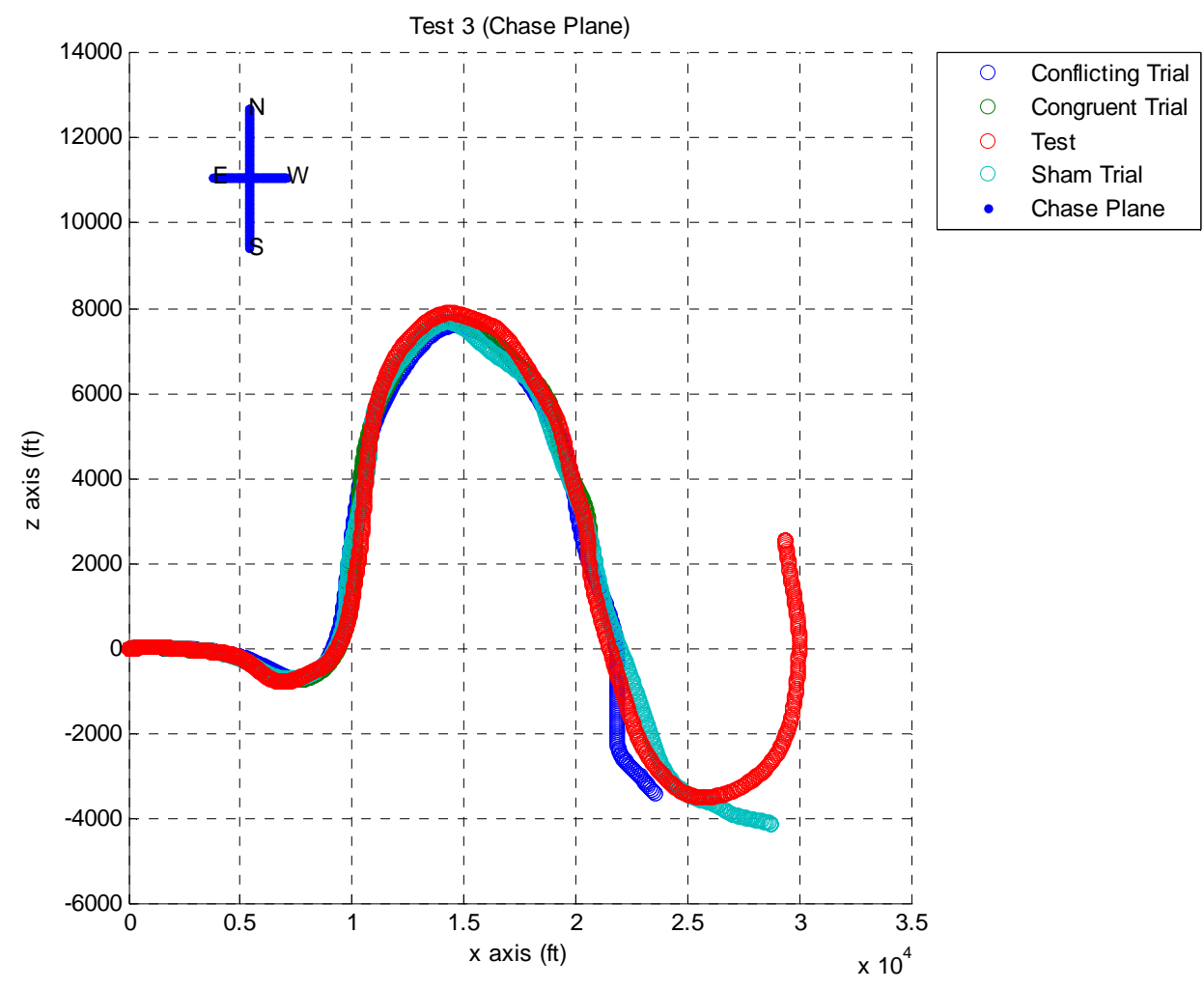

Figure 55 - Sample Test 3 (Chase Plane) 2-dimensional flight paths viewed from laying on the ground looking up.

The two-dimensional plot provides an easier way to visualize the path of the flight. In order to maintain the turns the aircraft must be banked. Figure 56 shows the RMS difference in roll angle between the chase plane and the subjects with the various stimulation profiles. For each stimulation profile the average RMS role deviation is represented by the light blue bar. 


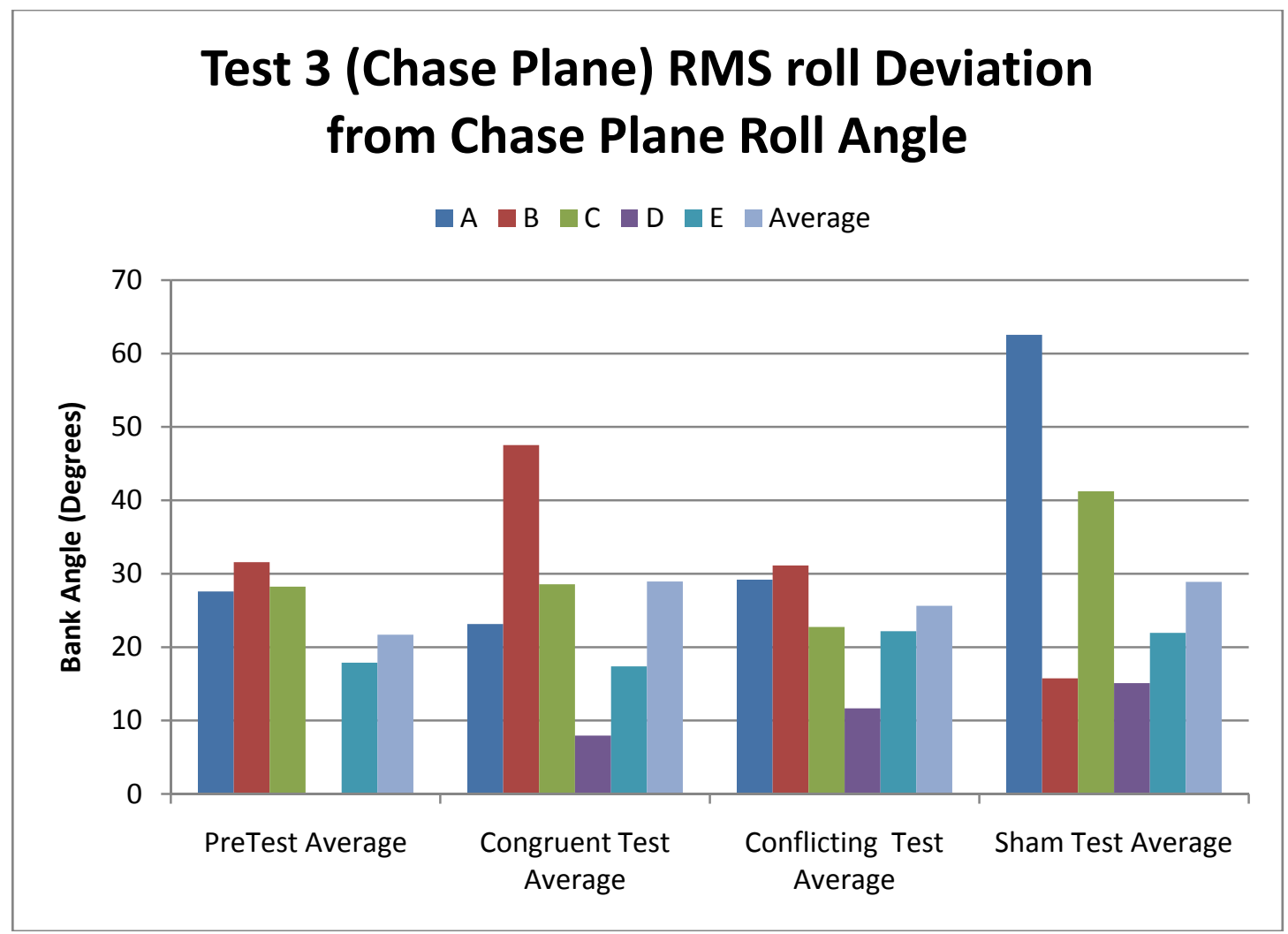

Figure 56 - RMS roll deviations for Test 3 (Chase Plane)

The statistical analysis with total RMS roll deviation values of test 3 as the dependent variable indicated that there is not enough statistical difference between the congruent, conflicting, and sham stimulations to exclude the possibility that the difference in total RMS roll deviation for each stimulation profile is a result of random sampling variability $(\mathrm{p}=0.630)$. The test had a power of 0.05 . 


\section{CHAPTER 7 : Conclusions}

The first objective of this experiment was to investigate whether GVS can be used as a test for individual susceptibility to motion sickness. The GVS did elicit rocking sensations in all of the subjects. The trend line in Figure 43 shows the motion sickness score increases as the rocking frequency increases. Two out of the three highest motion sickness scores over 100 did report feeling nausea shortly after the experiment. However, the data from Figure 43 shows that there was no relationship between the motion sickness score and the final sickness score reported after stimulation. GVS can evoke motion sickness, and it may be that the frequency of rocking was not fast enough to elicit motion sickness in most of the subjects.

The second objective of this experiment was to explore the effects of GVS coupled with a visual stimulus. This was done by coupling flight tasks with vestibular stimulations in response to roll data from a flight simulator. A comparison of subject flight task performance under congruent, conflicting, and sham stimulation profiles was performed.

The first test VS-A was designed to have minimal stimulations. The statistical analysis of the magnetic heading $(\mathrm{p}=0.162)$ shown in Figure 47 did not indicate any statistical differences between the congruent, conflicting, and sham stimulations that cannot be accounted for by chance or randomness in the testing. The low power (0.203) indicates that the sample size may have been too small to detect any significant differences. However, the lack of difference is expected because correct completion of the task should result in no stimulations. If the 
subject maintains a heading close to zero degrees north the 10 degree roll stimulation threshold is not exceeded.

The second test, VS-C, was designed to have at least four stimulations. During the test, the subject rolls past the 10 degree stimulation threshold twice when rolling right 30 degrees and the subject rolls past the -10 degree threshold twice when rolling left -30 degrees. The stimulation remains until they drop below the +/- 10 degree threshold. Figure 53 shows deviations from the expected +/- 30 degree bank angle. The statistical analysis of this data $(\mathrm{p}=0.346)$ also does not indicate any differences between the congruent, conflicting, and sham stimulations that cannot be accounted for by randomness involved with the testing. According to the data, the stimulations were not effective in stimulating the subjects to adjust their performance in completing the task. This may be because the test required the subjects to use the flight instruments to complete the task rather than visual cues. In addition, the low power (0.073) indicates that more subjects may need to be tested to detect any significant differences between the test conditions.

The final task was designed eliminate the dependence on the flight instruments by requiring the subject to follow a chase plane. Figure 54 shows the RMS roll deviations from the roll angle of the chase plane. The statistical analysis of the chase plane data $(\mathrm{p}=0.630)$ does not indicate any differences between the congruent, conflicting, and sham stimulations that cannot be accounted for by randomness involved with testing. The stimulations again were not effective in stimulating the subjects to adjust performance in completing tasks. The power of 
performance score (0.050) indicates that more subjects may need to be tested in order to detect a difference between the congruent, conflicting, and sham stimulations during the chase plane test.

The GVS induced motion sickness scores from this experiment did not show a strong relationship to the Kennedy Motion Sickness Questionnaire scores. The results did show an increase in sickness score with increasing frequency. The flight tests did not indicate statistical differences in the performance of flight tasks with congruent, conflicting, and sham stimulations. The statistics for the flight tasks did indicate that testing more subjects could show a difference in performance between the congruent, conflicting, and sham stimulations. Nine subjects were tested for the flight tasks in this experiment, but the data for four of them was incomplete as a result of the subject not being skilled enough to complete all of the flight tasks within two hours or experimenter errors while saving the data.

However, the feedback of the subjects throughout the testing provided valuable insight into action areas that would likely improve the effectiveness of GVS in both of these applications. During the motion sensitivity test, the subjects indicated that the stimulations felt like they were physically pushing them back and forth. The cathode stimulation typically provided a shock sensation the subjects associated with the pushing. During the second flight task, VS-C, many of the subjects began to sense when the stimulation would happen. After banking the aircraft a few times in VS-C, many of the subjects had figured out that the stimulation happened after they passed the threshold. How does this 
feedback relate to improving the effectiveness of the stimulations? First the shocking sensation needs to be minimized or eliminated. The shock sensation not only provides a distraction due to slight discomfort, but the shock also serves as a reference for the subject to interpret their experiences, ultimately effecting there flight performance. The subjects are expecting to feel pushed after the shock for the motion sensitivity test and they expect to feel shocked after they pass the 10 degree threshold in the flight task. Reducing or eliminating the shock would likely remove both of these factors associated with these tests. The second improvement is to increase the amount of orientation generated by the GVS. The device stimulates orientation during the initial current pulse, but, according to verbal feedback from the subjects, it does not provide significant orientation sensations after that the initial pulse. This feedback provides the framework for future work to improve the experiments. 


\section{CHAPTER 8 : Future work}

In response to the two areas of improvement, pilot testing was performed to lay a foundation for future work. The pilot testing began by attempting to decrease the sensation of current entering the body and to increase the orientation sensation by altering the electrical stimulation waveform. The logarithmic and linear ramping waveforms shown in Figure 58 and Figure 59 were the waveforms tested in six subjects, one of which was tested in the flight simulator experiment. In order to test these waveforms additional functionality was added to the custom GVS Software.

\subsection{GVS GUI Script Functionality}

To further test the effect of the ramping waveforms, functionality was added to the custom GVS software to automatically program scripts into the GVS device for roll right, roll left, pitch forward, and pitch backward. The new program allows the user to define the amplitude, the number of steps to reach the amplitude, the duration of each step in 25 millisecond increments, and how long to hold the pulse after reaching the final amplitude. Figure 57 shows the new program GUI with the added scripting functionality. 


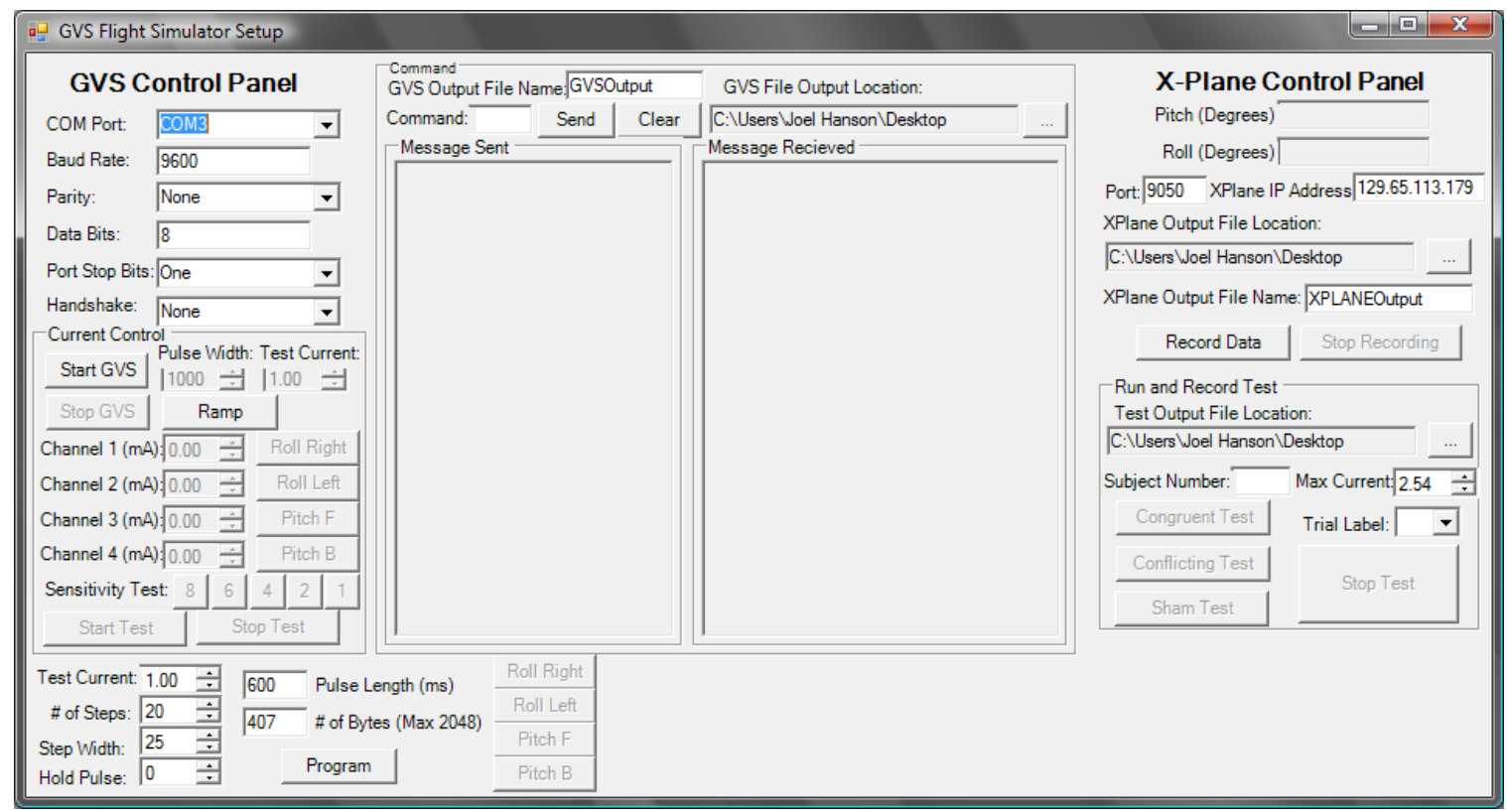

Figure 57 - New program graphic user interface

The memory on the GVS can hold up to 2048 bytes. The program automatically calculates the number of bytes used in the script and disables the "program button" if this 2048 is exceeded. The program also automatically calculates the length of the stimulation pulse in milliseconds.

\subsection{Ramping Functions}

Figure 58 was generated directly from the software, which shows the pulse can be updated about every 30 milliseconds. Figure 59 was generated from a script loaded onto the GVS device. The script can update the waveform every 25 milliseconds. 


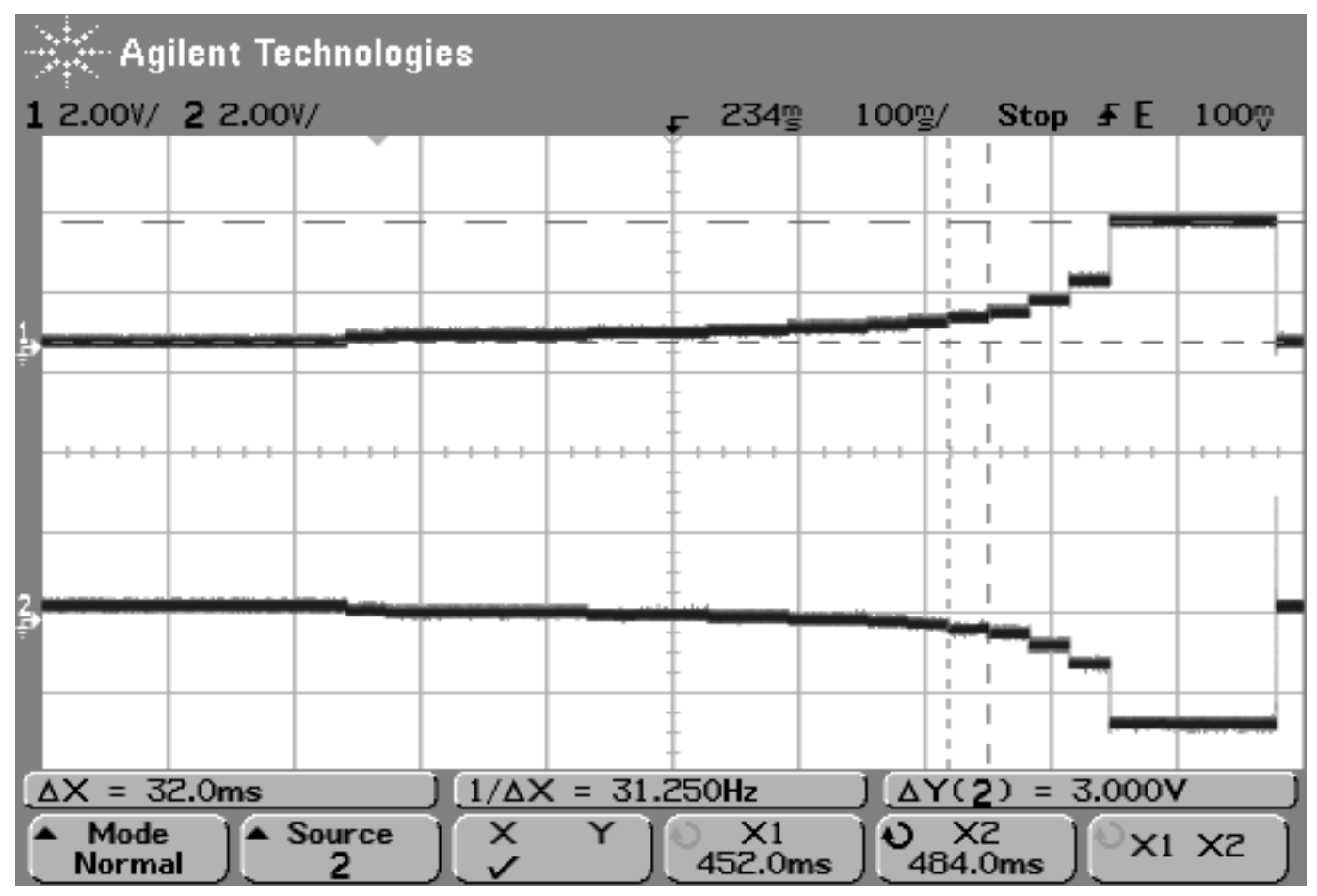

Figure 58 - GVS Software generated logarithmic waveform stimulation. The waveform had a 1.5 mA current across a $2 \mathrm{~K} \Omega$ resistor.

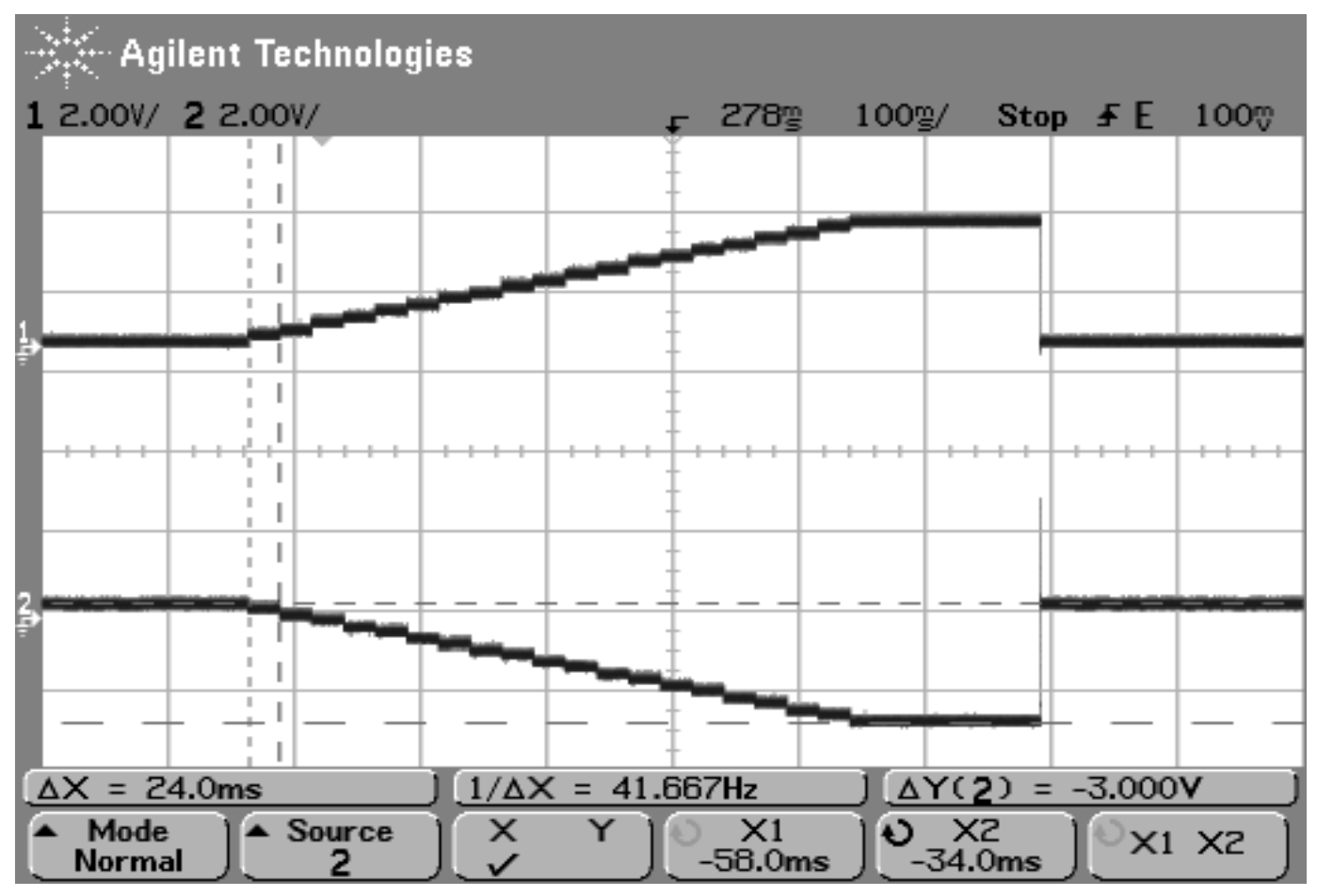

Figure 59-Script generated linear ramping waveform stimulation. The waveform had a $1.5 \mathrm{~mA}$ current across a $2 \mathrm{~K} \Omega$ resistor. 
Each of the waveforms did reduce the shocking sensation. However the linear ramping waveform not only lessened the shocking sensation, but it generated a significant increase in the orientation sensation for six out of six subjects. The orientation sensation is strong enough that subjects could not ignore the sensation even while sitting in a chair with the lights on, which was not true of the square pulse used in the experiments. The ramping waveforms reduced the shocking sensation enough for six out of six subjects to comfortably receive currents beyond $2 \mathrm{~mA}$. In the motion susceptibility and flight test the max comfortable current used was $1.5 \mathrm{~mA}$. However, many subjects were not able to reach $1.5 \mathrm{~mA}$ because the shock was too uncomfortable or too many of their head muscles were being stimulated. The ramping functions increased the current necessary to stimulate any muscles on the head to over $2 \mathrm{~mA}$. The square pulse waveform would begin stimulating muscles on the head as low as $0.7 \mathrm{~mA}$. The larger range of current levels is important because all of the pilot subjects had increased sensations as the current magnitude was increased.

The exponential waveform provided a sharper rolling sensation than the linear ramping function. It would be useful to implement a test that charts the sensations of subjects to various ramping waveforms with different current magnitudes. The information from this type of test could be used to program more realistic stimulations during the aircraft flight. 


\subsection{Additional improvement ideas}

Another factor that increased the stimulation perception was removing the delay in the stimulations applied to each ear. This was done after contacting the GVS device manufacturer to find out if there was another command to set the current on all of the electrodes with one message sent to the device. Programming scripts into the device permits both sides of the head to receive stimulations simultaneously.

Another idea for improving the flight simulator test is adjusting the frequency of stimulations. Figure 60 shows the cycles of a nerve cell.
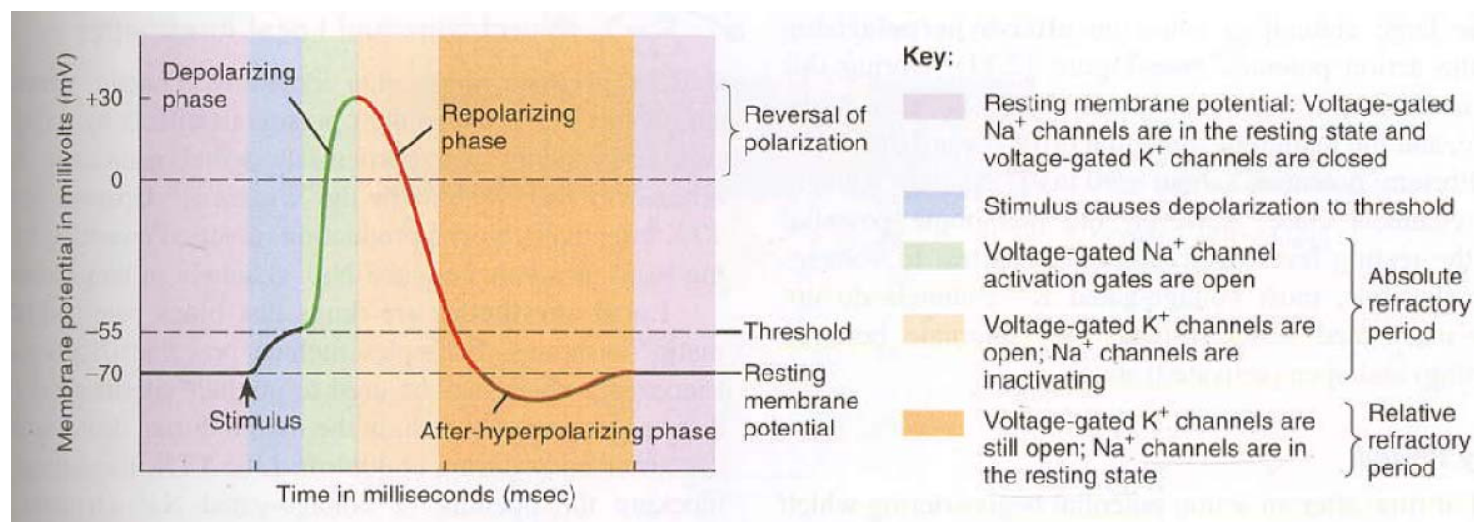

Figure 60 - Cycles of a nerve cell [24]

The idea would be to stimulate the vestibular nerves with slow pulse trains for a slow turn and as fast as the nerve can recover for a fast turn. With a small pulse width, larger roll angles could have fast large ramping current pulse stimulations. The smaller roll angles could have less frequent slower ramping current pulses. 


\subsection{Closing Comments}

The increased orientation sensations associated with the ramping waveforms provide much stronger and consistent orientation responses while reducing the sensation of current entering the body. With lower currents pilot subjects were easily made dizzy, which is a significant improvement for the motion sensitivity test. In addition, further testing of the sensations generated through the various ramping functions provides data to better program the flight simulator. Through programming the GVS script, these improved stimulations can be updated up to every 25 milliseconds with more realistic sensations. The ramping function can potentially be used to stimulate quick and slow roll changes resulting from the aircraft, which provides stronger sensation to the subjects. Stronger more realistic orientation sensations during the flight tasks are desirable to better test the performance of the subjects under congruent, conflicting, and sham stimulation profiles. 


\title{
Appendix I - Informed Consent Form for Cal Poly Research
}

\author{
Informed Consent Form for Cal Poly Research Participant Code \#
}

INFORMED CONSENT TO PARTICIPATE IN A STUDY ENTITLED:

Galvanic Vestibular Stimulation in Flight Simulators

A research project on human response to Vestibular Stimulation is being conducted by_Joel Hanson and Dr. Lynne Slivovsky of the Electrical Engineering Department, and Dr. Brian Self of the Mechanical Engineering Department, at Cal Poly San Luis Obispo. Erin Best of the Kinesiology department has been trained on the procedures and will be assisting the researchers on the tests. The purpose of this research is to study the effects of galvanic vestibular stimulation on disorientation. Figure 1 below shows the electrodes used for stimulation. Ultimately this technique may be used to enhance disorientation training during simulated or actual flight, which may reduce the number of aircraft accidents resulting from spatial disorientation. Reducing the number of accidents may save the military money, and more importantly save pilots' lives. The results may also be used to test pilots vestibular response prior to in flight testing.

You are being asked to take part in this study by providing your medical history including history of vestibular, neuromuscular or musculoskeletal disorder, and completing questionnaires related to susceptibility to motion sickness. This will take about 20 minutes. If appropriate, you will then be asked to allow no more than 2.6 milliamps (mA) of current to stimulate your vestibular system (which includes the inner ears and is responsible for your sensations of body orientation.) The testing will be done over two days which will be separated by a minimum 48 hours to ensure the integrity of the test. The first day will take about 90 minutes and will test the vestibular response to galvanic vestibular stimulation coupled with a flight simulator. The second day of testing will take place in a dark room and will take about 45 minutes. The amounts of current can be perceived by you and will affect your sensations of balance and orientation in space, but are below the threshold at which you might feel pain. As shown in figure 2 below, the electrodes will be placed on both mastoid processes behind your left and right ear, on your forehead, and in the middle of the back of your neck. Prior to the stimulations you will receive a pre-test consisting of current levels from $0 \mathrm{~mA}$ to no more than $2.6 \mathrm{~mA}$, which is the maximum level that the device can output. The stimulation will be applied through electrodes behind your ears as shown in Figure 1 and Figure 2 below. This will ensure you are comfortable with the stimulation current levels and want to proceed with the experiment. Throughout the experiment you will be provided with the power button on the GVS that will allow you to cease electrical stimulation at any moment. Before leaving you must pass three standardized California Highway Patrol sobriety tests to ensure you are no longer disoriented. In all, your involvement in these experimental procedures will take approximately 3 hours. Please be aware that you are not required to participate in this research and you may discontinue your participation at any time without penalty. 
The possible risks associated with participation in this study include slight discomfort or skin irritation, disorientation, involuntary eye movement, dizziness, nausea, or vomiting. These risks will be minimized by providing good electrical contact, keeping currents small, and maintaining communication at all times. If you exhibit any signs of vestibular disorientation mentioned above for greater than 24 hours after participating in the study, please contact the researchers and seek medical attention. Please be aware that any health care costs that result from the experiment will be the responsibility of the subject.

Your confidentiality will be protected as all data collected will be referenced only by numeric code, not name. The only connection between the numeric code used to distinguish the data and the name of the subject will be this signed form with the numeric code written on the top. These forms will be kept locked in the office of Dr. Lynne Slivovsky to ensure confidentiality. All electronic data will be stored on a password protected computer and destroyed after completion of the project.

If you have questions regarding this study or would like to be informed of the results when the study is completed, please feel free to contact Joel Hanson (925) 2854575 , or his faculty advisors Dr. Slivovsky at (805) 756-5383 or Dr. Self at (805) 7567993. If you have questions or concerns regarding the manner in which the study is conducted, you may Dr. Steve Davis, Chair of the Cal Poly Human Subjects Committee, at 756-2754, sdavis@calpoly.edu, or Dr. Susan Opava, Dean of Research and Graduate Programs, at 756-1508, sopava@calpoly.edu.

If you agree to voluntarily participate in this research project as described, please indicate your agreement by signing below. Please keep one copy of this form for your reference, and thank you for your participation in this research.

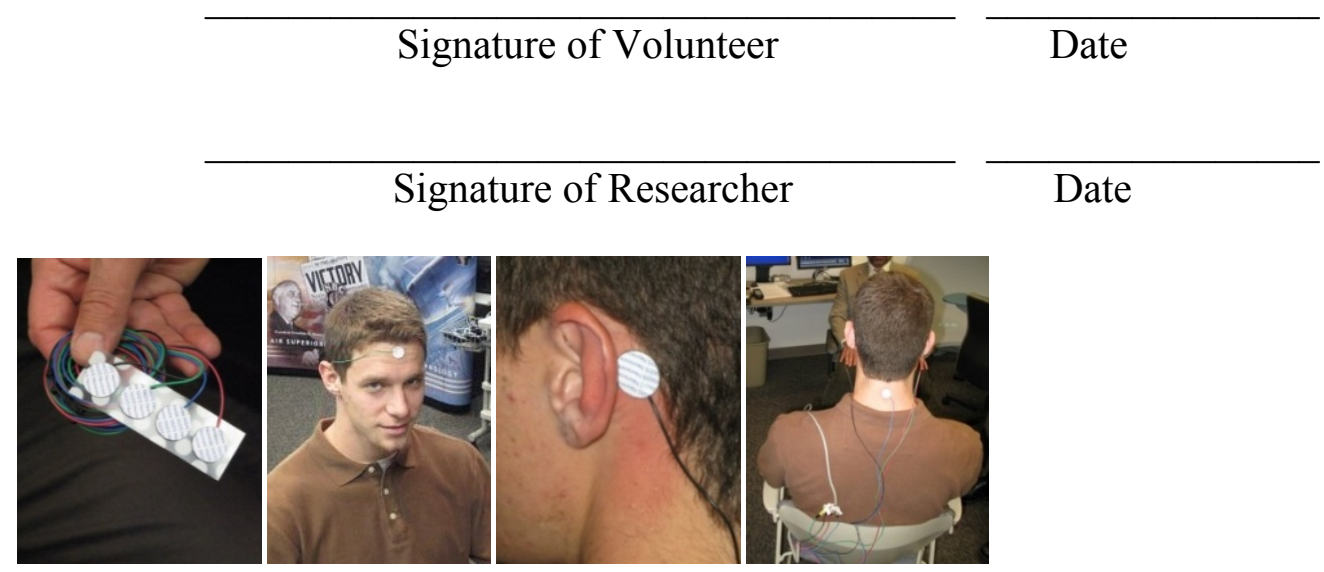

\section{Date}

Figure 1 - Sample Electrodes
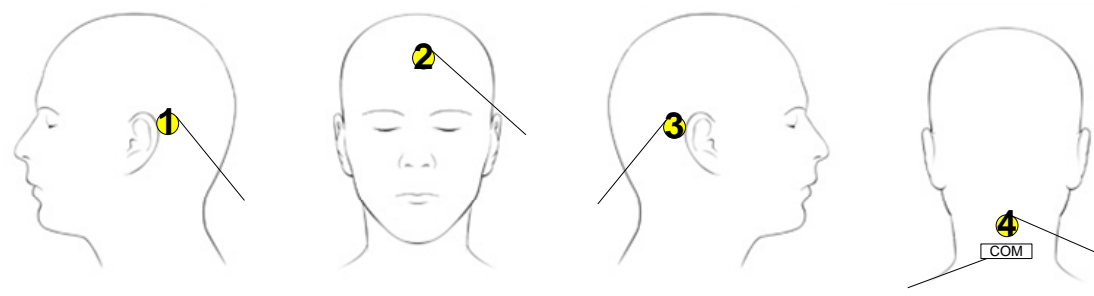

Figure 2 - Electrode Placement 
Code \#

\section{Appendix II - Motion Sickness Screening Questionnaire}

1. Gender:

Male

Female

2. Age:

3. In general, how susceptible to motion sickness are you?

Extremely___ Very___ Moderately___ Minimally

Not at all

Section A: Childhood Experience only (before 12 years of age)

For each of the following types of transportation or entertainment please indicate:

4. As a Child (before age 12), how often you Traveled or Experienced (tick boxes):

\begin{tabular}{|l|c|c|c|c|}
\hline & Never & 1-4 Trips & 5-10 Trips & 11 or More \\
\hline Cars & & & & \\
\hline Buses or Coaches & & & & \\
\hline Trains & & & & \\
\hline Aircraft & & & & \\
\hline Small Boats & & & & \\
\hline Ships, e.g. Channel Ferries & & & & \\
\hline Swings & & & & \\
\hline Roundabouts: playgrounds & & & & \\
\hline Big Dippers, Funfair Rides & & & & \\
\hline
\end{tabular}

5. As a Child (before age 12), how often you Felt Sick or Nauseated (tick boxes):

\begin{tabular}{|l|c|c|c|c|c|}
\hline & Never & Rarely & Sometimes & Frequently & Always \\
\hline Cars & & & & & \\
\hline Buses or Coaches & & & & & \\
\hline Trains & & & & & \\
\hline Aircraft & & & & & \\
\hline Small Boats & & & & & \\
\hline Ships, e.g. Channel Ferries & & & & & \\
\hline Swings & & & & & \\
\hline Roundabouts: playgrounds & & & & & \\
\hline Big Dippers, Funfair Rides & & & & & \\
\hline
\end{tabular}

6. As a Child (before age 12), how often you Vomited (tick boxes):

\begin{tabular}{|l|c|c|c|c|c|}
\hline & Never & Rarely & Sometimes & Frequently & Always \\
\hline Cars & & & & & \\
\hline Buses or Coaches & & & & & \\
\hline Trains & & & & & \\
\hline Aircraft & & & & & \\
\hline Small Boats & & & & & \\
\hline Ships, e.g. Channel Ferries & & & & & \\
\hline Swings & & & & & \\
\hline Roundabouts: playgrounds & & & & & 4 \\
\hline Big Dippers, Funfair Rides & & & & \\
\hline
\end{tabular}


Section B: Your Experience over the past 10 Years (approximately)

For each of the following types of transportation or entertainment please indicate:

7. Over the past 10 years, how often you Traveled or Experienced (tick boxes):

\begin{tabular}{|l|c|c|c|c|}
\hline & Never & $1-4$ Trips & $5-10$ Trips & 11 or More \\
\hline Cars & & & & \\
\hline Buses or Coaches & & & & \\
\hline Trains & & & & \\
\hline Aircraft & & & & \\
\hline Small Boats & & & & \\
\hline Ships, e.g. Channel Ferries & & & & \\
\hline Swings & & & & \\
\hline Roundabouts: playgrounds & & & & \\
\hline Big Dippers, Funfair Rides & & & \\
\hline
\end{tabular}

8. Over the past 10 years, how often you Felt Sick or Nauseated (tick boxes):

\begin{tabular}{|l|c|c|c|c|c|}
\hline & Never & Rarely & Sometimes & Frequently & Always \\
\hline Cars & & & & & \\
\hline Buses or Coaches & & & & & \\
\hline Trains & & & & & \\
\hline Aircraft & & & & & \\
\hline Small Boats & & & & & \\
\hline Ships, e.g. Channel Ferries & & & & & \\
\hline Swings & & & & & \\
\hline Roundabouts: playgrounds & & & & & 4 \\
\hline Big Dippers, Funfair Rides & & & & \\
\hline
\end{tabular}

9. Over the last 10 years, how often you Vomited (tick boxes):

\begin{tabular}{|l|c|c|c|c|c|}
\hline & Never & Rarely & Sometimes & Frequently & Always \\
\hline Cars & & & & & \\
\hline Buses or Coaches & & & & & \\
\hline Trains & & & & & \\
\hline Aircraft & & & & & \\
\hline Small Boats & & & & & \\
\hline Ships, e.g. Channel Ferries & & & & & \\
\hline Swings & & & & & \\
\hline Roundabouts: playgrounds & & & & & \\
\hline Big Dippers, Funfair Rides & & & & & \\
\hline
\end{tabular}

10. Do you have any history of the following:

Vestibular disorder: Yes No

Epilepsy: Yes No

Neurological or Musculo-skeletal problems: Yes No 
11. Have you consumed any alcohol in the last 24 hours? Yes

No

12. Is there any other reasons why it would not be appropriate for you to serve as a subject in this experiment? Yes No

(i.e. Current or a history of head injury, vision problems, hearing problems, vertigo, and psychological disorders that might cause an adverse reaction (e.g., major anxiety), as well as current illness(es), use of certain medications including psychoactive drugs, and pregnancy)

I certify that my responses to this questionnaire are accurate.

Name Signature Date 
Code \#

\section{Appendix III - Test Administrator Checklist}

Overall sense of motion sickness:

Subjects responses to overall sensation:

(0: no symptoms; 1: any symptoms, however slight; 2 : mild symptoms, e.g., stomach awareness but not nausea; 3 : mild nausea; 4: mild to Symptom moderate nausea; 5 : moderate nausea but can continue; 6 : moderate

8 seconds

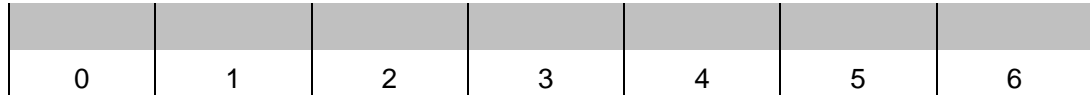

6 seconds

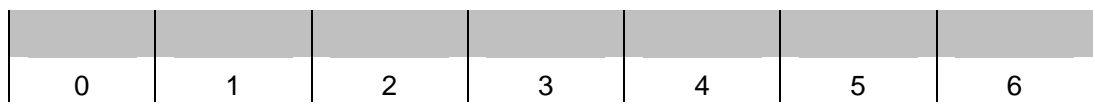

4 seconds

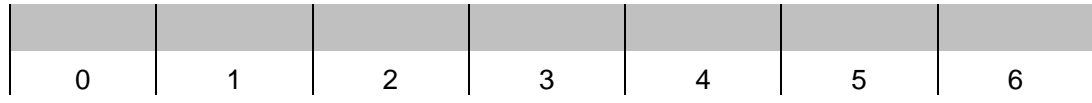

2 seconds

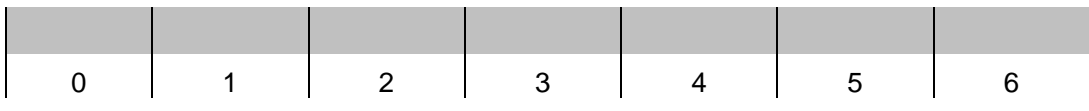

1 seconds

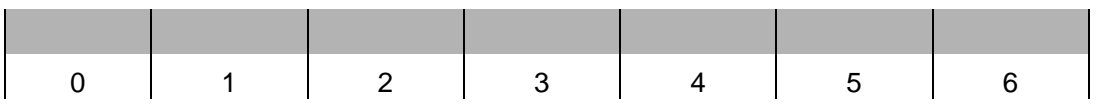

1. Please note make any additional notes about the subjects responses. 
Code \#

\section{Appendix IV - Simulator Sickness Questionnaire}

Please answer the following questions about your experience as accurate as possible:

1. While in the simulator please check how often you felt:

\begin{tabular}{|l|l|l|l|l|}
\hline & Never & Rarely & Frequently & Always \\
\hline General discomfort & & & & \\
\hline Fatigue & & & & \\
\hline Headache & & & & \\
\hline Eyestrain & & & & \\
\hline Difficulty focusing & & & & \\
\hline Increased Salvation & & & & \\
\hline Sweating & & & & \\
\hline Nausea & & & & \\
\hline Difficulty concentrating & & & & \\
\hline Fullness of Head & & & & \\
\hline Blurred Vision & & & & \\
\hline Dizziness (eyes open) & & & & \\
\hline Dizziness (eyes closed) & & & & \\
\hline Vertigo & & & & \\
\hline Stomach Awareness & & & & \\
\hline Burping & & & & \\
\hline
\end{tabular}

2. Overall rate your sensation of disorientation.

Subjects responses to overall sensation:

(0: no symptoms; 1: any symptoms, however slight; 2 : mild symptoms, e.g., stomach awareness but not nausea; 3 : mild nausea; 4: mild to Symptom moderate nausea; 5 : moderate nausea but can continue; 6 : moderate

Overall nausea and want to stop)

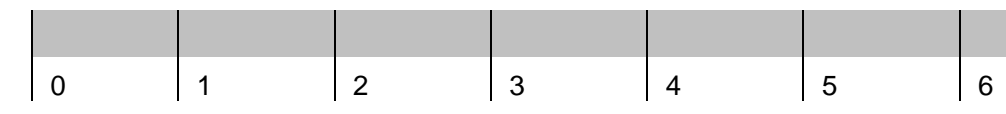

3. Please note make any additional notes about your experience. 


\section{Reference List}

[1] Bent, Leah R., Bradford J. McFadyen, Veronique F. Merkley, Paul M. Kennedy , and $\mathrm{J}$ T. Inglis. "Magnitude Effects of Galvanic Vestibular Stimulation on the Trajectory of Human Gait." Nueroscience Letters (2000): 157-160. 7 May 2008.

[2] Barnett-Cowan, Michael, and Lawrence R. Harris. "Percieved timing of vestibular stimulation relative to touch, light, and sound." (2009). Multisensory Integration Laboratory. 22 May 2009.

[3] Cacioppo, John T., Louis G. Tassinary, and Gary G. Berntson. Hand of Psychophysiology. 2nd ed. Camgridge: Cambridge University, 2000.

[4] Chatterjee, Indira, Ding Wu, and Om P. Gandhi. "Human Body Impedance and Threshold Currents for Contact Hazard Analysis in VLF-MF Band." IEEE Transactions on Biomedical Engineering 33 (1986). IEEE Explore. Google Scholar. 2 May 2008.

[5] Coulter, Gary R., and Gregory L. Vogt. "The Effects of Space Flight on the Human Vestibular System." Weboflife.Nasa.Com. Nasa. 9 June 2008 $<$ http://weboflife.nasa.gov/learningResources/vestibularbrief.htm>.

[6] Day, B. L., A. Severac Cauquil, L. Bartolomei, M. A. Pastor, and I. N. Lyon. "Human body - segment tilts induced by galvanic stimulation: a vestibularly driven balance protection mechanism." Journal of Physiology 500 (1997): 661-72.

[7] Dzurkova, O., and F. Hlavacka. "Velocity of Body Lean Evoked by Leg Muscle Vibration Potentiate the Effects of Vestibular Stimulation on Posture." Physiological Research 56 (2007): 829-832. Medline. Cal Poly. 16 Apr. 2008

[8] Fitzpatrick, Richard C., and Brian L. Day. "Probing the Human Vestibular System with Galvanic Stimulation." Journal of Applied Physiology 96 (2004): 2301-2316. Medline. Cal Poly State University. 17 May 2008.

[9] Francoise, Marie, and Tardy Gervet. "Effects of Galvanic Vestibular Stimulation on Perception of Subjective Vertical in Standing Humans." Perceptual \& Motor Skills 86 (1998): 1155-1157. Academic Search Elite. Cal Poly, San Luis Obispo. 08 Mar. 2008.

[10] Ghanim, Z., J. C. Lamy, A. Lackmy, V. Achache, N. Roche, A. Penicaud, S. Meunier, and R. Katz. "Effects of galvanic mastoid stimulation in seated human subjects." Journal of Applied Physiology 106 (2009): 893-903.

[11] George, Crampton H. Motion and Space Sickness. CRC P, 1990. 0-451. 
[12] Golding, John F. "Motion sickness susceptibility questionnaire revised." Brain Research Bulletin, 47 (1999): 507-16. ScienceDirect. 23 Oct. 2008.

[13] Griffin, Michael J., and Barnaby E. Donohew. "Motion Sickness with Fully RollCompensated Lateral Oscillation: Effect of Oscillation Frequency." Aviation, Space, and Environmental Medicine 80: 94-101.

[14] Guerraz, Michel, and Brian L. Day. "Expectation and the Vestibular Control of Balance." Journal of Cognitive Neuroscience 17 (2005): 463-69.

[15] Guyton, Arthur C. Medical Physiology. 7th ed. Philadelphia: W.B. Saunders Company, 1986.

[16] Kennedy, Robert S., Jennifer E. Fowlkes, Kevin S. Berbaum, and Michael G. Lilienthal. "Use of a Motion Sickness History Questionnaire for Prediction of Simulator Sickness." Aviation, Space, and Environmental Medicine (1992): 588593.

[17] Lobel, Elie, Justus F. Kleine, Denis Le Bihan, Anne Leroy-Willig, and Alain Berthoz. "Functional MRI of Galvanic Vestibular Stimulation." The American Phsiological Society (1998).

[18] Malcik, Vladmir. "Performance Decrement in a Flight Simulator Due to Galvanic Vestibular Organ and Its Validity for Success in Flight Training." Aerospace Medicine (1968): 941-943. 15 Mar. 2008.

[19] Medical instrumentation application and design. New York: Wiley, 1998.

[20] Prausnitz, Mark R. "The effects of electrical current applied to skin: A review for tansdermal drug delivery." Advanced Drug Delivery Reviews 18 (1996): 395-425.

[21] Scinicariello, Anthony P., Kenneth Eaton, J. Timothy Inglis, and J. J. Collins. "Enhancing human balance control with galvanic vestibular stimulation." Biological Cybernetics (2001). 17 June 2009.

[22] "Spatial Disorientation Confusion That Kills." www.asf.org. 2004. AOPA Safety Foundation. 19 Mar. $2008<$ http://www.aopa.org/asf/publications/sa17.pdf>.

[23] The Highway Safety Desk Book. National Highway Safety Traffic Administration. 6 Sept. $2008<$ http://www.nhtsa.dot.gov/people/injury/enforce/deskbk.html\#sfst>.

[24] Tortora, Gerard J., and Sandra R. Grabowski. Principles of Anatomy and Physiology. 10th ed. Hoboken: John Wiley \& Sons Inc, 2003. 546-556. 
[25] United States. U.S. Department of Transportation. Federal Aviation Administration. Instrument Flying Handbook. Oklahoma City: United States Department of Transportation, 2007. Print.

[26] Watson, Shaun R. D., Agatha E. Brizuela, Ian S. Curthoys, James G. Colebatch Hamish G. Macdougall, and Michael D. Halmagyi. "Maintained Ocular Torsion Produced by Bilateral and Unilateral Galvanic (DC) Vestibular Stimulation in Humans." Experimental Brain Research 122 (1998): 453-458. 08 Mar. 2008.

[27]Zink, R, S.f. Bucher, A. Weiss, Th. Brandt, and M. Dieterich. "Effects of Galvanic Vestibular Stimulation on Otolithic and Semicircular Canal Eye Movements and Percieved Vertical." Electroencephalography and Clinical Neurophysiology 107 (1998): 200-205. Science Direct. Google Scholar. 25 Apr. 2008. 